\title{
Distant radio galaxies and their environments
}

\author{
George Miley • Carlos De Breuck
}

Received: 19 October 2007 / Published online: 29 January 2008

(C) The Author(s) 2008

\begin{abstract}
We review the properties and nature of luminous high-redshift radio galaxies (HzRGs, $z>2$ ) and the environments in which they are located. HzRGs have several distinct constituents which interact with each other-relativistic plasma, gas in various forms, dust, stars and an active galactic nucleus (AGN). These building blocks provide unique diagnostics about conditions in the early Universe. We discuss the properties of each constituent. Evidence is presented that HzRGs are massive forming galaxies and the progenitors of brightest cluster galaxies in the local Universe. HzRGs are located in overdense regions in the early Universe and are frequently surrounded by protoclusters. We review the properties and nature of these radio-selected protoclusters. Finally we consider the potential for future progress in the field during the next few decades. A compendium of known HzRGs is given in an appendix.
\end{abstract}

Keywords Radio galaxies $\cdot$ High-redshift $\cdot$ Massive galaxies $\cdot$ Clusters

\section{Contents}

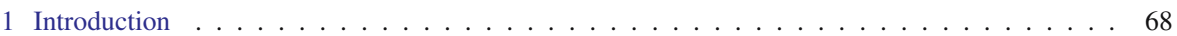

1.1 Scope of this article-HzRGs . . . . . . . . . . . . . . . . . . . . . 69

1.2 History . . . . . . . . . . . . . . . . . . . . . . . . 69

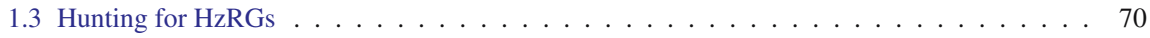

1.4 Redshift distribution of HzRGs . . . . . . . . . . . . . . . . . . . . . . . . . 72

G. Miley $(\varangle)$

Sterrewacht, Leiden University, Postbus 9513, 2300RA Leiden, The Netherlands e-mail: miley@strw.leidenuniv.nl

C. De Breuck

European Southern Observatory, Karl-Schwarzschild-Strasse 2, 85748 Garching, Germany e-mail: cdebreuc@eso.org 
1.5 Constituents of HzRGs . . . . . . . . . . . . . . . . . . . . . . . . . 72

2 Relativistic plasma . . . . . . . . . . . . . . . . . . . . . . . 74

2.1 HzRGS and low-redshift radio galaxies . . . . . . . . . . . . . . . . . . 75

2.2 Radio sizes and morphologies: size as an evolutionary clock . . . . . . . . . . . . . 75

2.3 Radio size vs redshift correlation . . . . . . . . . . . . . . . . . . . . . . . . 77

2.4 Radio spectral index vs redshift correlation . . . . . . . . . . . . . . . . . . . . . 77

2.5 Non-thermal X-ray emission . . . . . . . . . . . . . . . . . . . . . . . 79

3 Gas and dust . . . . . . . . . . . . . . . . . . . . . . . . 80

3.1 Hot ionised gas-radio depolarisation . . . . . . . . . . . . . . . . . 80

3.2 Warm ionised gas . . . . . . . . . . . . . . . . . . . . . . . 82

3.3 Neutral gas . . . . . . . . . . . . . . . . . . . . . . . . 88

3.4 Molecular gas . . . . . . . . . . . . . . . . . . . . . . . . . . 90

3.5 Dust . . . . . . . . . . . . . . . . . . . . . 93

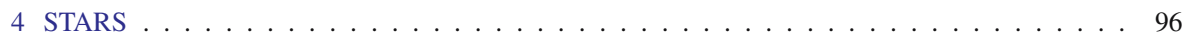

4.1 Old stars: HzRGs as the most massive galaxies . . . . . . . . . . . . . . . . . . . . . 97

4.2 Young stars-UV absorption lines . . . . . . . . . . . . . . . . . . . . . . . . 99

4.3 The alignment effect-jet-induced star formation . . . . . . . . . . . . . . . . . . . . . 99

5 Active galactic nucleus $(\mathrm{AGN}) \ldots \ldots \ldots \ldots \ldots \ldots$

5.1 Hidden or dormant quasar-The Unification Church . . . . . . . . . . . . . . . . . . 102

5.2 Supermassive black hole-powerhouse of the AGN . . . . . . . . . . . . . . . . . 103

5.3 Extinction of the "dinosaurs" . . . . . . . . . . . . . . . . . . . . . . . . . . . 104

6 Nature of HzRG hosts-massive forming galaxies . . . . . . . . . . . . . . . . . . . . . 104

6.1 The Spiderweb Galaxy —a case study . . . . . . . . . . . . . . . . . . . . . . . . . 106

7 Environment of HzRGS-protoclusters . . . . . . . . . . . . . . . . . . . . . . . . 107

7.1 Finding protoclusters using HzRGs . . . . . . . . . . . . . . . . . . . . . 108

7.2 Properties of radio-selected protoclusters . . . . . . . . . . . . . . . . . . . 109

7.3 Are radio-selected protoclusters typical $\ldots \ldots \ldots \ldots \ldots \ldots \ldots$

7.4 Protocluster evolution . . . . . . . . . . . . . . . . . . . . . . . . 113

8 Conclusions and future . . . . . . . . . . . . . . . . . . . . . . . 115

Appendix: Known HZRGs . . . . . . . . . . . . . . . . . . . . . . . . . . . . . . . . . . . . 117

\section{Introduction}

Martin Harwit. "Yes. I think that if there were less requirement for theoretical justification for building a instrument that is very powerful, you would then have a better chance of making a discovery."

Bernard Burke. "The record of radio astronomy is clear. Quasars were not found by the desire to find black holes or by the need to find long-distance cosmological probes."

Proceedings of Greenbank Workshop on Serendipitous Discovery in Radio Astronomy, 1983

Distant radio galaxies are among the largest, most luminous, most massive and most beautiful objects in the Universe. They are energetic sources of radiation throughout most of the electromagnetic spectrum. The radio sources are believed to be powered by accretion of matter onto supermassive black holes in the nuclei of their host galaxies. Not only are distant radio galaxies fascinating objects in their own right, but they also have several properties that make them unique probes of the early Universe. 


\subsection{Scope of this article-HzRGs}

Our review will be restricted to galaxies that have redshifts, $z>2$ and radio luminosities at $500 \mathrm{MHz}$ (rest frame) $\mathrm{L}_{500 \text { (rest) }}>10^{27} \mathrm{~W} \mathrm{~Hz}^{-1}$. Radio emission from such objects has a steep non-thermal spectrum, is collimated and is usually extended by tens of kiloparsecs. There are very few known $\mathrm{z}>2$ radio galaxies associated with compact flat-spectrum radio sources.

This definition is somewhat arbitrary. Luminous distant radio galaxies have properties that are different from less powerful radio galaxies at low redshifts and the properties of radio galaxies change gradually with luminosity and redshift. Likewise, $z>2$ quasars that are associated with steep-spectrum extended radio emission have many similar properties to our distant radio galaxies (see Sect. 5.1) and are located in similar environments. However, without some restriction, our task would not have been tractable.

For conciseness, we shall frequently refer to such high-redshift radio galaxies as "HzRGs". McCarthy (1993) gave an extensive review of this topic in 1993. Since then there have been several workshops on high-redshift radio galaxies, whose proceedings have been published, including Amsterdam (Röttgering et al. 1999b), Leiden (Jarvis and Röttgering 2003) and Granada (Villar-Martín et al. 2006a,b). Thousands of refereed papers dealing with distant radio galaxies and related topics have been published during the last fifteen years. Although our goal is to be comprehensive, some personal bias will inevitably have entered in selecting the topics and literature. Our apologies for this.

The structure of this review is as follows. We shall first set the scene by reviewing the history of the field. Then we shall describe techniques used for finding HzRGs and discuss how HzRGs are distributed in redshift. Following an overview of the diverse constituents of HzRGs, we shall discuss each HzRG component in detail. The nature of HzRGs and their role in the general evolution of galaxies will then be covered. We shall present evidence that HzRGs are the massive progenitors of dominant cluster galaxies in the local Universe and that they are located in forming galaxy clusters. The properties of these radio-selected protoclusters are then reviewed. Finally, we shall discuss some of the exciting prospects for future HzRG research. An appendix includes a list of known HzRGs at the time of writing (October 2007).

\subsection{History}

The study of distant radio galaxies has progressed in several phases:

(i) Infancy. Mid-1940s to mid-1960s: After the discovery that Cygnus A is associated with a faint distant galaxy (Baade and Minkowski 1954), radio astronomy became one of the most important tools of observational cosmology. Redshifts of up to $z=0.45$ were measured for galaxies associated with radio sources (Minkowski 1960). Two fundamental breakthroughs were made towards the end of this period. First, quasistellar radio sources or "quasars" were discovered, with much larger redshifts even than radio galaxies. Second, it was demonstrated that the space density of radio sources 
varies with cosmic epoch, an observation that sounded the death knell for the Steady State cosmology

(ii) Childhood. Mid-1960s to mid-1980s: This can be termed the "Spinrad era". Hy Spinrad devoted a considerable amount of his time, and that of the 120-inch telescope at Lick, to measuring the redshifts of faint radio galaxies by means of long photographic exposures. With considerable effort, he pushed the highest radio galaxy redshift out to $z \sim 1$ (Spinrad 1976; Spinrad et al. 1977; Smith and Spinrad 1980). At that time, it was not realised that radio sources interact with their optical hosts and the different wavelength regimes were usually studied in isolation from one another. Radio sources were regarded as interesting high-energy exotica that were useful for pinpointing distant elliptical galaxies. They were not believed to play an important role in the general scheme of galaxy evolution. During this period the paradigm that radio sources are powered by accretion of matter onto rotating black holes was developed.

(iii) Teens. Mid-1980s to mid-1990s: The replacement of photographic techniques by CCDs revolutionised optical astronomy. Large numbers of very distant radio galaxies were discovered with redshifts out to $z \sim 5$ (HzRGs). The surprising discovery that the radio sources and the optical host galaxies are aligned showed that there must be considerable interaction between the radio sources and their host galaxies. The role that orientation could have in determining observed properties was realised. "Orientation unification" that explained differences in the observed types of active galaxies as merely due to the viewing angle of the observer became a popular interpretative "do-it-all".

(iv) Maturity. Mid-1990s to the present: New efficient techniques for finding distant galaxies were developed. These included photometric searches for "dropout" objects with redshifted Lyman break features and narrow-band searches for objects with excess redshifted Lyman $\alpha$ fluxes. The advent of these new techniques placed the cosmological use of radio galaxies in a new perspective. Although radio astronomy lost its prime position as a technique for finding the most distant galaxies, it became clear that luminous radio galaxies play a highly important role in the evolution of galaxies and the emergence of large scale structure. The discovery of a relation between the masses of elliptical galaxies and the inferred masses of nuclear black holes led to the conclusion that all galaxies may have undergone nuclear activity at some time in their histories. This brought nuclear activity into the mainstream of galaxy evolution studies.

\subsection{Hunting for HzRGs}

Appendix A contains a compendium of 178 radio galaxies with $z>2$ known to the authors. This list is the result of more than two decades of hard work. The relatively small number of known HzRGs illustrates both their rarity and the difficulty of detecting them.

Finding distant radio galaxies involves a multistage process. 
- The first step (e.g. Röttgering et al. 1994; Blundell et al. 1998; De Breuck et al. 2000b, 2004b) is to filter out probable HzRGs from the huge number of radio sources contained in low-frequency radio surveys (e.g. Condon et al. 1998). The usual criteria used for filtering likely candidates are (i) radio colours-extremely steep spectra (Sect. 2.4) and (ii) small angular sizes (Sect. 2.3).

- In the second step, relatively bright nearby objects are discarded, by comparing the radio positions with existing wide-field shallow surveys at optical and near-infrared wavelengths.

- The third step is to refine the positions of the remaining radio sources to arcsecond accuracy, thereby facilitating their identification with faint galaxies. Until the 1990s, radio identifications were made using optical CCD data (e.g. Röttgering et al. 1995; McCarthy et al. 1996). However, after sensitive near-IR detectors became available, the near-IR $K$-band was found to be more efficient for identifying distant radio sources (e.g. Jarvis et al. 2001a,b, 2004; De Breuck et al. 2002, 2004b; Cruz et al. 2006). More than 94\% of bright radio sources are identified down to $K=22$. Furthermore, the $K$-band magnitude of an HzRG provides a first indication of its redshift, by means of the $K-z$ relationship (see Sect. 4.1).

- The fourth step is to carry out spectroscopic observations of the HzRG candidates with large optical and near-infrared telescopes. These are needed to determine redshifts from the wavelengths of HzRG emission lines (e.g. Röttgering et al. 1997; DeBreuck et al. 2001; De Breuck et al. 2006; Jarvis et al. 2001b; Bornancini et al. 2007) (see Sect. 3.2).

The main limitation on finding HzRGs has been the scarcity of available observing time on large optical/IR telescopes for the last stage in the process. Because candidates are located too far apart in the sky to permit multi-object spectroscopy, tedious long-slit spectroscopy on many individual fields is required to determine the redshifts.

Although radio selection ensures that there is no a priori selection against dust properties (e.g. extinction), there are other observational selection effects that introduce bias into the redshift distributions of HzRGs and the list given in the appendix. The first obvious source of bias is that the determination of a spectroscopic redshift is dependent on being able to observe bright emission lines with ground-based telescopes. The primary line for such redshift measurement (e.g. McCarthy, 1993) is Ly $\alpha \lambda 1216 \AA$ (typical equivalent widths of several hundred $\AA$ ). Useful additional lines are CIV $\lambda 1549 \AA$, HeII $\lambda 1640 \AA$ and CIII] $\lambda 1909 \AA$ (equivalent widths in excess of $\sim 60 \AA$ ). There is a so-called "redshift desert" $1.2<z<1.8$ (e.g. Cruz et al. 2006), where [OII] $\lambda 3727 \AA$ is too red and Ly $\alpha \lambda 1216 \AA$ is too blue to be easily observed from ground-based optical telescopes. Objects located within the redshift desert are thus under-represented in samples of HzRGs.

A second source of bias is redshift incompleteness. A small fraction of radio sources, ( $\sim 4 \%)$ is not identified to $K \sim 22$ and about a third of those with K-band identifications do not show any emission or absorption lines, even after long exposures on 8-10 m class optical/IR telescopes. These objects either (i) have emission lines that 
are hidden by substantial dust obscuration (e.g. De Breuck et al. 2001; Reuland et al. 2003a), (ii) are located at such large redshifts that Ly $\alpha$ and other bright emission lines fall outside the easily observable spectral windows, or (iii) are peculiar in that they radiate no strong emission lines.

\subsection{Redshift distribution of HzRGs}

HzRGs are extremely rare. Luminous steep-spectrum radio sources associated with HzRGs have typical luminosities of $\mathrm{L}_{2.7 \mathrm{GHz}}>10^{33} \mathrm{erg} \mathrm{s}^{-1} \mathrm{~Hz}^{-1} \mathrm{ster}^{-1}$. The number density of radio sources with this luminosity in the redshift range $2<z<5$ is a few times $10^{-8} \mathrm{Mpc}^{-3}$, with large uncertainties (Dunlop and Peacock 1990; Willott et al. 2001; Venemans et al. 2007).

Although HzRGs are sparsely distributed in the early Universe, such objects are almost non-existent at low redshifts. The co-moving space density of luminous steepspectrum radio sources increases dramatically by a factor of 100-1000 between $0 \leq$ $z<2.5$ and then appears to flatten out (Willott et al. 2001; Jarvis et al. 2001c). Although there are huge uncertainties in the evolution of the luminosity function at higher redshifts, no significant cut-off in space density has yet been observed.

The redshifts at which the space density of radio sources is maximum correspond to a crucial era in the evolution of the Universe. It coincides with the epoch when (i) luminous quasars also appear to have had their maximum space density (e.g. Pei 1995; Fan et al. 2001) (ii) star formation was rampant and more than an order of magnitude larger than the present (Madau et al. 1998; Lilly et al. 1996; Schiminovich et al. 2005) and (iii) galaxy clusters were forming, but were not yet gravitationally bound structures.

The question of why the most luminous radio sources (and the most luminous quasars) became extinct in the local Universe is an intriguing one that is still not understood (Sect. 5.3).

\subsection{Constituents of HzRGs}

Radio galaxies have several distinct emitting components which provide diagnostics about various physical constituents of the early Universe. A list of known HzRG building blocks is given in Table 1, together with a summary of techniques used to study them. Also included are a list of the resultant diagnostics, some useful relevant references and our best estimate for the typical mass of the component in HzRGs. We caution the reader to thoroughly examine the assumptions inherent in deriving each of the diagnostics. Although, these assumptions are needed in order to reach some conclusions about the nature of HzRGs, it is important never to forget that they exist and to be sceptical about the "houses of cards" that are frequently involved.

Because they are highly luminous and (unlike quasars) spatially resolvable from the ground, most components of HzRGs provide important diagnostic information about the spatial distributions of processes within HzRGs and their environment. 
Table 1 Constituents of distant radio galaxies

\begin{tabular}{|c|c|c|c|c|}
\hline Constituent & Observable & Typical diagnostics & Refs. $^{\mathrm{a}}$ & $\begin{array}{l}\text { Mass } \\
\left(\mathrm{M}_{\odot}\right)\end{array}$ \\
\hline \multirow[t]{2}{*}{ Relativistic plasma } & Radio continuum & $\begin{array}{l}\text { Magnetic field, age, ener- } \\
\text { getics, pressure, particle } \\
\text { acceleration. Jet collima- } \\
\text { tion and propagation }\end{array}$ & 1,2 & \\
\hline & $\mathrm{X}$-ray continuum & $\begin{array}{l}\text { Magnetic field, equiparti- } \\
\text { tion, pressures }\end{array}$ & $3,4,1$ & \\
\hline \multirow{2}{*}{$\begin{array}{l}\text { Hot ionised gas } \\
T_{e} \sim 10^{7}-10^{8} \mathrm{~K} \\
n_{e} \sim 10^{-1.5} \mathrm{~cm}^{-3}\end{array}$} & $\begin{array}{l}\text { Radio (de)polari- } \\
\text { sation }\end{array}$ & Density, magnetic field, & 1 & $10^{11-12}$ \\
\hline & $\mathrm{X}$-rays & Temperature, density mass & & \\
\hline \multirow[t]{2}{*}{$\begin{array}{l}\text { Warm ionised gas } \\
T_{e} \sim 10^{4}-10^{5} \mathrm{~K} \\
\mathrm{n}_{e} \sim 10^{0.5-1.5} \mathrm{~cm}^{-3}\end{array}$} & $\begin{array}{l}\text { UV-optical } \\
\text { emission lines }\end{array}$ & $\begin{array}{l}\text { Temperature, density, kine- } \\
\text { matics, mass, ionisation, } \\
\text { metallicity, filling factor }\end{array}$ & $\begin{array}{l}5,6,7 \\
8\end{array}$ & $10^{9-10.5}$ \\
\hline & $\begin{array}{l}\text { Nebular } \\
\text { continuum }\end{array}$ & SED contamination & 9,10 & \\
\hline \multirow[t]{2}{*}{$\begin{array}{l}\text { Cool atomic gas } \\
T_{S} \sim 10^{3} \mathrm{~K} \\
n(\mathrm{HI}) \sim 10^{1} \mathrm{~cm}^{-3}\end{array}$} & HI absorption & $\begin{array}{l}\text { Kinematics, column den- } \\
\text { sities, spin temperature, } \\
\text { sizes, mass }\end{array}$ & 11,8 & $10^{7-8}$ \\
\hline & $\begin{array}{l}\text { UV-optical } \\
\text { absorption lines }\end{array}$ & $\begin{array}{l}\text { Kinematics, mass,column } \\
\text { densities, metallicity }\end{array}$ & $\begin{array}{l}8,12, \\
13,14\end{array}$ & \\
\hline $\begin{array}{l}\text { Molecular gas } \\
T \sim 50-500 \mathrm{~K} \\
\mathrm{n}\left(\mathrm{H}_{2}\right)>10^{2} \mathrm{~cm}^{-3}\end{array}$ & (Sub)millimetre lines & Temperature, density, mass & 15 & $10^{10-11}$ \\
\hline \multirow[t]{2}{*}{$\begin{array}{l}\text { Dust } \\
T \sim 50-500 \mathrm{~K}\end{array}$} & $\begin{array}{l}\text { UV-optical } \\
\text { polarisation }\end{array}$ & $\begin{array}{l}\text { Dust composition, scatter- } \\
\text { ing, mass, hidden quasar }\end{array}$ & 16,17 & $10^{8-9}$ \\
\hline & $\begin{array}{l}\text { (Sub)millimetre con- } \\
\text { tinuum }\end{array}$ & $\begin{array}{l}\text { Temperature, mass, heating } \\
\text { source }\end{array}$ & 18 & \\
\hline $\begin{array}{l}\text { Old stars } \\
t>1 \mathrm{Gyr}\end{array}$ & $\begin{array}{l}\text { Optical to near IR } \\
\text { continuum }\end{array}$ & Age, mass, formation epoch & 19 & $10^{11-12}$ \\
\hline \multirow[t]{2}{*}{$\begin{array}{l}\text { Young stars } \\
t<0.5 \mathrm{Gyr}\end{array}$} & UV-optical & $\begin{array}{l}\text { Star formation rates, ages, } \\
\text { history }\end{array}$ & 20,8 & $10^{9-10}$ \\
\hline & $\operatorname{Ly} \alpha$ & Star formation rate & 20 & \\
\hline $\begin{array}{l}\text { Quasar (hidden or dor- } \\
\text { mant) }\end{array}$ & $\begin{array}{l}\text { UV-optical polarisa- } \\
\text { tion } \\
\text { broad lines }\end{array}$ & Luminosity & 21,22 & \\
\hline $\begin{array}{l}\text { Supermassive black hole } \\
\text { (SMBH) }\end{array}$ & $\begin{array}{l}\text { Extended radio, } \\
\text { Quasar }\end{array}$ & Formation, evolution & 23,24 & $\sim 10^{9}$ \\
\hline
\end{tabular}

${ }^{a}$ References: 1 = Miley (1980), 2 = Klamer et al. (2006), 3 = Felten and Morrison (1966), 4 = Schwartz (2002), 5 = Osterbrock and Ferland (2006), $6=$ Groves et al. (2004a), $7=$ Groves et al. (2004b), $8=$ Dopita and Sutherland (2003), $9=$ Aller (1984), $10=$ Dickson et al. (1995), $11=$ Morganti (2006), 12= van Ojik et al. (1997a), $13=$ Binette et al. (2000), $14=$ Binette et al. (2006), $15=$ Downes et al. (1993), $16=$ Cimatti et al. (1993), $17=$ Vernet et al. (2001b), $18=$ Reuland et al. (2004), $19=$ Seymour et al. (2007), 20 = Madau et al. (1998), 21 = Cimatti et al. (1993), $22=$ Vernet et al. (2001b), $23=$ Blandford (2001), 24 = Peacock (1999)

The fact that the different constituents are present in the same objects and that the interrelationships and interactions between them can be studied make distant radio galaxies unique laboratories for probing the early Universe. 


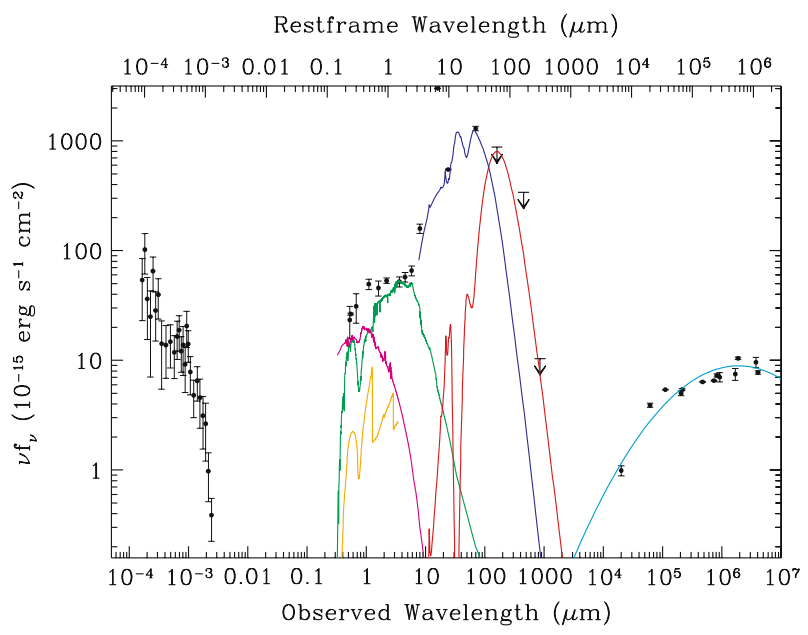

Fig. 1 Spectral energy distribution (SED) of the continuum emission from the HzRG 4C23.56 at $z=2.5$, illustrating the contributions from the various constituents [from De Breuck et al. in preparation]. Coloured lines show the decomposition of the SED into individual components, under many assumptions. Cyan radio synchrotron (Sect. 2); Black Absorbed non-thermal X-ray AGN (Sect. 2.5); Yellow nebular continuum (Sect. 3.2.6); Blue AGN-heated thermal dust emission (Sect. 3.5); Red Starburst-heated dust emission (Sect. 3.5); Green Stars (Sect. 4); Magenta scattered quasar (Sects. 3.5.2 and 5). The addition of the overlapping modeled components fits the SED well

As can be seen in Table 1, several constituents of HzRGs are inferred to be extremely massive, including old stars (up to $\sim 10^{12} \mathrm{M}_{\odot}$ ), hot gas (up to $\sim 10^{12} \mathrm{M}_{\odot}$ ) and molecular gas (up to $\sim 10^{11} \mathrm{M}_{\odot}$ ).

Figure 1 shows the spectral energy distribution (SED) of a typical HzRG from radio to X-ray wavelengths, together with a decomposition into various observable HzRG constituents-relativistic plasma, gas and dust, stars and the active galactic nuclei (AGN). We shall discuss each of these building blocks individually in Sects. 2, 3, 4 and 5. Note from Fig. 1 that disentangling the various components in the optical and the infrared is difficult and the results are often extremely model dependent and several alternative solutions may be equally consistent with the available multi-wavelength data.

\section{Relativistic plasma}

"All things are double, one against another" Ecclesiasticus (xlvii. 24)

"The problem is how much to stress the sameness of twins and how much to emphasise their differences.", Baby and Child Care, Benjamin Spock, 1946

Shortly after the discovery of extragalactic radio sources, their non-thermal spectra and polarisations led to the conclusion that their emission is produced by synchrotron radiation from a relativistic plasma. The large radio luminosities together with the lifetimes of the synchrotron-emitting electrons imply total energies of $\geq 10^{60} \mathrm{erg}$ (e.g. Miley 1980). These huge energies, the collimation on sub-parsec scales and the simi- 
larity of orientation between the compact and overall radio structures led to the conclusion that the collimated relativistic beams are produced by rotating supermassive black holes (SMBHs) located at the centres of the host galaxies. The SMBHs are postulated to have rotation axes aligned with the radio source axes. The radio sources are powered through gravitational energy from material accreting onto the SMBHs, that is converted into kinetic energy of the collimated relativistic jets (see Sect. 5.2).

\subsection{HzRGS and low-redshift radio galaxies}

Extragalactic radio sources can be classified according to their sizes.

- Most radio sources detected in low-frequency radio surveys $(v \lesssim 2 \mathrm{GHz}$ ) are "extended", with projected linear sizes ranging from several tens of kiloparsec to several megaparsecs, i.e. much larger than those of their optical galaxy hosts. Extended extragalactic radio sources have non-thermal spectra, with typical spectral indices at low redshifts of $\alpha \sim-0.7$, with flux proportional to $v^{\alpha}$. An example is Cygnus A in Fig. 3.

- Most radio sources detected in high-frequency radio surveys are "compact", with typical sizes of $<1 \mathrm{pc}$ and relatively flat spectral indices $(\alpha>-0.4)$.

- A small fraction of sources have linear sizes between $\sim 100 \mathrm{pc}$ and a few kpc. These sources have peaked spectra that are self absorbed at low frequencies $(<\mathrm{a}$ few $\mathrm{GHz}$ ) and are variously known as "peakers", gigahertz-peaked spectrum (GPS) sources or compact steep-spectrum (CSS) sources.

Galaxies and quasars host extragalactic radio sources of all three classes, with extended radio sources predominantly identified with galaxies and compact sources mainly associated with quasars.

HzRGs generally host extended extragalactic radio sources and differ in several properties from low-redshift radio galaxies. At larger redshifts the typical radio luminosities increase, the typical sizes decrease (Sect. 2.3) and the typical radio spectra steepen (Sect. 2.4). The host galaxies of HzRGs differ from the low-redshift radio galaxy hosts in (i) the presence of emission-line halos (Sect. 3.2, (ii) increased clumpiness of the continuum emission (Sect. 4.3) and (iii) alignments of the radio sources with both the emission-line halos (Sect. 3.2.3) and the UV/optical galaxy continuum emission (Sect. 4.3) (Fig. 2).

\subsection{Radio sizes and morphologies: size as an evolutionary clock}

The radio structures of HzRGs have been studied by Carilli et al. (1997) and Pentericci et al. (2000b). Both articles describe observations of 37 radio galaxies with $z>2$ using the VLA at 4.7 and $8.2 \mathrm{GHz}$, at resolutions down to $0.25^{\prime \prime}$. In accordance with their large radio luminosities, most HzRGs are "Fanaroff-Riley Class II" radio sources (Fanaroff and Riley 1974), with double structures, edge-brightened mophologies and one or more hot-spots located at the extremities of their lobes (Miley 1980; Carilli et al. 1994, 1997; Pentericci et al. 2000b). In general HzRGs do not have appreciable flat-spectrum core components at their nuclei, but the use of 


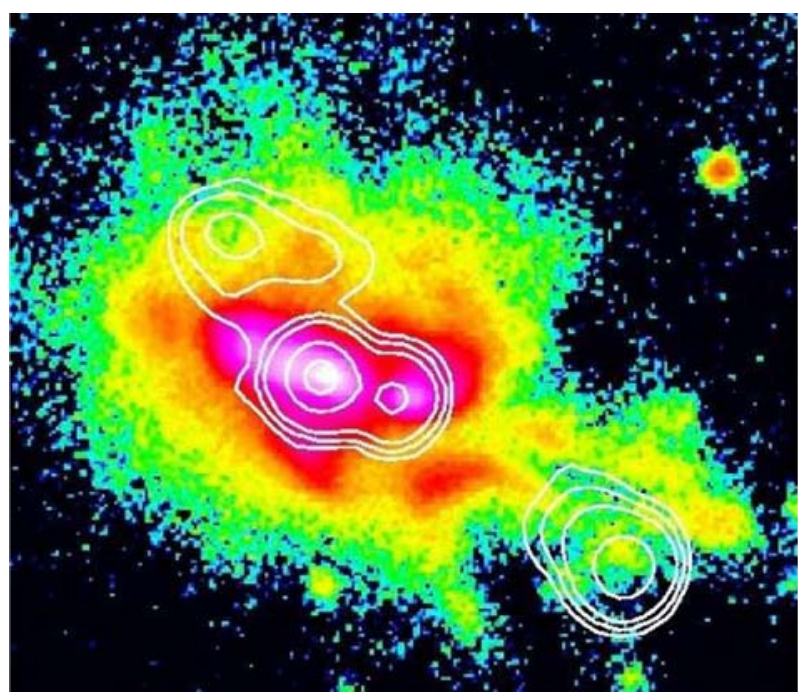

Fig. 2 Radio structure of 4C41.17 at $z=3.8$ [from van Breugel and Reuland, private communication]. See also Reuland et al. (2003b). Contours obtained at $1.4 \mathrm{GHz}$ with the VLA are superimposed on a Keck narrow-band image in redshifted $\operatorname{Ly} \alpha$, showing the warm ionised gas (Sect. 3.2). The radio angular size of $\sim 13^{\prime \prime}$ corresponds to a projected linear size of $\sim 90 \mathrm{kpc}$. The radio spectrum of this object is shown in Fig. 3c. Figure 5 illustrated the radio rotation measure of the brightest radio component and Fig. 15 is a higher resolution radio contour map of the central region

the ultra-steep spectrum criterion in searching for HzRGs discriminates against finding HzRGs with flat-spectrum cores. Standard minimum energy assumptions (Miley 1980) give typical minimum pressures in these hotspots of a few $\times 10^{-9} \mathrm{dyn} \mathrm{cm}^{-2}$ and corresponding magnetic field strengths of a few hundred $\mu \mathrm{G}$.

There have been a few VLBI observations of fine-scale structure in the lobes of extended radio sources associated with HzRGs (Gurvits et al. 1997; Cai et al. 2002; Pérez-Torres and De Breuck 2005; Pérez-Torres et al. 2006 ). A component of size $\sim 65 \mathrm{pc}$ has been detected several kpc from the nucleus of $4 \mathrm{C} 41.17$ at $z=3.8$ (Pérez-Torres et al. 2006 ). Consideration of the energetics suggests that the radio component is associated with a gas clump of mass $M_{B} \geq 1.5 \times 10^{8} M \odot$. Intriguingly, this is typical for the masses of "predisrupted clumps" invoked as the progenitors of globular clusters (Fall and Rees 1977). Investigating fine scale radio structure in HzRGs and using the radio jet interactions to probe the interstellar medium in the early Universe are likely to be an important field of study for the more sensitive longbaseline interferometers that are presently under construction, such as e-Merlin and the e-EVN.

There are several correlations between the sizes of radio sources and other properties (Röttgering et al. 2000). First, for 3C sources at lower redshifts, there is a relation with optical morphology. Smaller radio sources consist of several bright optical knots aligned along the radio axes, while larger sources are less clumpy and aligned (Best et al. 1998). Second, there is a relation with emission line properties. Smaller radio sources have generally lower ionisations, higher emission line fluxes and broader 
line widths than larger sources (Best et al. 1999). Third, there is a connection with Ly $\alpha$ absorption. HzRGs with smaller radio sizes are more likely to have Ly $\alpha$ absorption than larger sources (Sect. 3.3.2) (van Ojik et al. 1997a). Taken together, these correlations are consistent with an evolutionary scenario in which radio size can be used as a "clock" that measures the time elapsed since the start of the radio activity.

Note that the large sizes of radio sources (usually several tens of kpc) imply that the nucleus of host galaxies has been undergoing activity for at least $>10^{5}$ years (light travel time across the source) and up to $>10^{8}$ years (assuming that the jets advance at a few hundred $\mathrm{km} / \mathrm{s}$ ). Hence we know that AGN associated with extended radio sources must be long-lived. This is not the case for radio-quiet or compact radio quasars, that may only have been active quasars for a few $\times 10^{2}$ years.

\subsection{Radio size vs redshift correlation}

It has been known since the nineteen sixties that there is a statistical decrease of the angular size of radio sources with redshift (Miley 1968). More recent work includes measurement of the angular size-redshift relation for luminous extended radio sources (Nilsson et al. 1993; Neeser et al. 1995; Daly and Guerra 2002), quasars (Buchalter et al. 1998) and compact radio sources (Gurvits et al. 1999).

Over the years there have been many valiant attempts to use the angular-redshift relation to derive information about the geometry of the Universe (e.g. determination of $q_{0}$ and $\Omega_{0}$ ) and even to set constraints on dark energy (Podariu et al. 2003). However, there are many observational selection effects involved. Furthermore, it is difficult to disentangle varying geometry of the Universe from effects due to physical evolution of the radio sources, their host galaxies and the surrounding ambient medium. For example, the sizes of radio sources can be expected to decrease at larger redshifts due to a systematic increase in density of the ambient medium. Also the energy density of the cosmic microwave background increases as $(1+z)^{4}$, substantially enhancing inverse Compton losses. This will tend to extinguish radio sources earlier in their lives, making them on average smaller.

In summary, the many non-cosmological effects that influence the angular sizeredshift relation have made it impossible to draw robust conclusions about cosmology from such considerations.

\subsection{Radio spectral index vs redshift correlation}

One of the most intriguing properties of the relativistic plasma in HzRGs is the strong correlation that exists between the steepness of radio source spectra and the redshift of the associated radio galaxies (Tielens et al. 1979; Blumenthal and Miley 1979). Radio sources with very steep spectral indices at low frequencies $\lesssim 1 \mathrm{GHz}$ tend to be associated with galaxies at high redshift (e.g. Fig. 3). This empirical correlation between radio spectral steepness and redshift has proved to be an efficient method for finding distant radio galaxies. Most known HzRGs (Table 3) have been discovered through following up those radio sources with the steepest ten percentile of radio spectra (spectral index, $\alpha \sim-1$ ). 

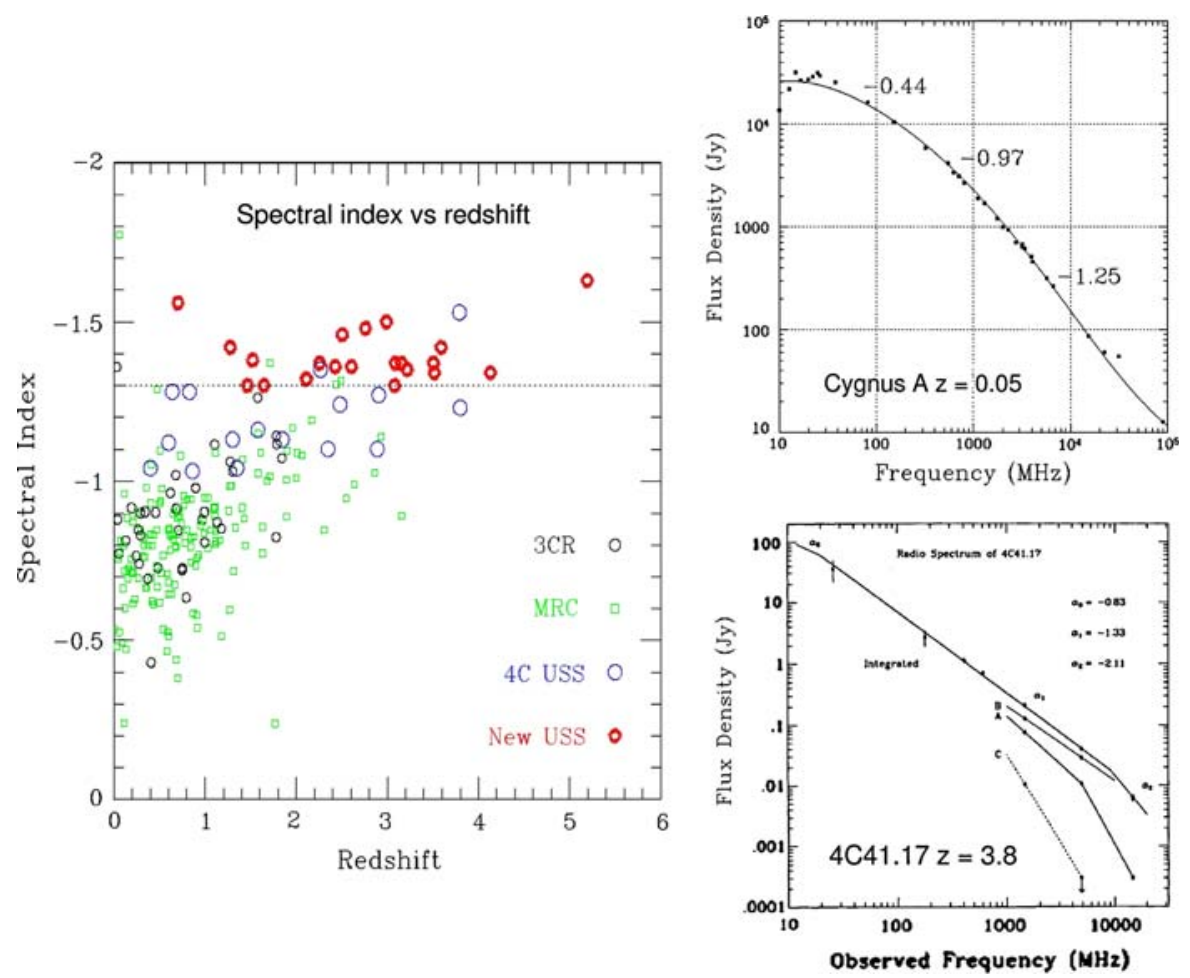

Fig. 3 Left Plot of radio spectral index versus redshift, showing that more distant sources have steeper spectra [from De Breuck et al. (2000b)]. Above right Radio spectrum of the luminous radio galaxy Cygnus A at $z=0.05$, showing spectral curvature. Bottom right Radio spectrum of the HzRG 4C41.17 and its various components [from Chambers et al. (1990)]. This is still one of the most well determined spectra of a HzRG at low frequencies. Note the absence of significant spectral curvature

The conventional explanation for the $z \sim \alpha$ correlation is that it is the result of a concave radio spectrum (see Cygnus A in Fig. 3), coupled with a radio K-correction. For higher redshifts, observations of sources at a fixed observed frequency will sample emission at higher rest-frame frequencies where the concave spectrum becomes steeper. The most important mechanisms for making the radio spectra concave are synchrotron and inverse Compton losses at high frequencies (e.g. Klamer et al. 2006) and synchrotron self-absorption at low frequencies.

Although there is evidence from radio colour-colour plots that systematic concave curvature is present in the radio spectra (e.g. Bornancini et al. 2007), such an explanation alone is insufficient to explain the observed correlation. The radio spectra of many distant luminous radio galaxies are not concave at the relevant frequencies. The radio source with the most accurately determined spectrum over a wide frequency range is $4 \mathrm{C} 41.17$ at $z=3.8$ (bottom right in Fig. 3). This source has an extremely straight spectrum between $40 \mathrm{MHz}$ and $5 \mathrm{GHz}$, the relevant frequency range for the $\mathrm{z} \sim \alpha$ correlation (Chambers et al. 1990). Although the spectrum steepens above $5 \mathrm{GHz}$, this is too high a frequency to contribute to the $z \sim \alpha$ correlation. Furthermore, a recent 
study by Klamer et al. (2006) showed that 33 of 37 sources in their SUMSS-NVSS sample have straight and not concave spectra between 0.8 and $18 \mathrm{GHz}$.

Two alternative effects have been proposed to explain the observed $z \sim \alpha$ relation. The first possibility is that the $z \sim \alpha$ relation is an indirect manifestation of a luminosity, $L \sim \alpha$ effect (Chambers et al. 1990; Blundell and Rawlings 1999).

Classical synchrotron theory predicts that continuous particle injection will result in a spectrum with a low-frequency cut-off, $v_{l}$, whose frequency depends on the source luminosity, $L$ according to $v_{l} \sim L^{-6 / 7}$. For a flux-limited sample, Malmquist bias will cause sources at higher redshift to have preferentially larger radio luminosities. Over the relevant frequency range, the $L^{-6 / 7}$ vs $v_{l}$ effect would therefore result in the observation of an $z \sim \alpha$ relation. However, it is unlikely that this luminosityspectrum relation is the correct explanation, or at least the whole story. Athreya and Kapahi (1998a) showed that a $z \sim \alpha$ correlation still persists even when samples are restricted to a limited range of $L$.

A second explanation is that some physical effect causes the spectral index to steepen with higher ambient density and that the ambient density increases with redshift (Athreya and Kapahi 1998a; Klamer et al. 2006). For example, putting the radio source in a denser environment would cause the upstream fluid velocity of the relativistic particles to decrease and a first-order Fermi acceleration process would then produce a steeper synchrotron spectrum. Recently Klamer et al. (2006) pointed out that such a mechanism would (i) result in both $z \sim \alpha$ and $L \sim \alpha$ correlations and (ii) provide a natural physical link between high-redshift radio galaxies and nearby cluster halos.

However, it is difficult to produce the observed $\mathrm{z} \sim \alpha$ relation from such a simple density-dependent effect alone. The clumpy UV/optical morphologies (e.g. Sect. 6.1) indicate that the density of gas around HzRGs is highly non-uniform and that the density is larger close to the nucleus than in the outer regions. Furthermore, the internal spatial variations of spectral index within individual source is observed to be smaller than the source to source variations of the integrated spectral indices (e.g. Carilli et al. 1994). If the ambient medium is highly non-uniform, how can one side of a radio source "know" that the other side has an uncommon ultra-steep spectrum? It is therefore likelier that the ultra-steep spectra are produced by some mechanism in which the spectral index is determined within the galaxy nucleus rather than by the environment at the locations of the radio lobes.

In summary, the origin of the $z \sim \alpha$ effect is still unclear and more detailed information is needed about the dependence of the radio spectrum on redshift. In the near future accurate measurements of low-frequency radio spectra of HzRGs with new facilities such as LOFAR will be important for such studies.

\subsection{Non-thermal X-ray emission}

Although X-ray measurements of HzRGs are sensitivity-limited, significant progress in the field has been made in the last decade using the Chandra and XMM X-ray telescopes. Extended X-ray emission has been detected from about a dozen high-redshift radio galaxies and radio-loud quasars (Carilli et al. 2002b; Fabian et al. 2003a,b; 
Scharf et al. 2003; Yuan et al. 2003; Belsole et al. 2004; Overzier et al. 2005; Blundell et al. 2006; Erlund et al. 2006; Johnson et al. 2007). The extended X-ray emission is typically elongated in the direction of the radio source, indicating that there is some physical link between the X-ray emission and the relativistic plasma.

Several mechanisms have been proposed for producing the extended X-ray emission. The one that is most widely suggested inverse Compton scattering (IC) of the cosmic microwave background (Fabian et al. 2003a; Scharf et al. 2003; Belsole et al. 2004; Erlund et al. 2006; Johnson et al. 2007). Because the density of CMB photons increase as $(1+z)^{4}$, IC scattering of the CMB becomes increasingly important at high redshift. Under the assumption that the X-ray emission is due to this process, comparison of the radio and X-ray luminosities (Felten and Rees 1969) yields magnetic field strengths consistent with equipartition (Belsole et al. 2004; Overzier et al. 2005; Johnson et al. 2007). Because the radiative lifetimes of radio synchrotronemitting electrons are shorter than the lifetimes of the X-ray emitting IC electrons, the IC emission traces older particles. In 4C 23.56 at $z=2.48$, the X-ray emission is observed to extend by $\sim 500 \mathrm{kpc}$ (Johnson et al. 2007), implying an energy in both relativistic and IC-emitting electrons of $\geq 10^{59} \mathrm{erg}$, an energy reservoir equivalent to $\sim 10^{8}$ supernovae.

Other processes that have been invoked to produce the extended X-ray emission include inverse-Compton up-scattering of synchrotron photons in the jet (synchrotron self-Compton emission-SSC) (Scharf et al. 2003) and thermal emission from shocks (Carilli et al. 2002b; Belsole et al. 2004) (see Sect. 3.1).

\section{Gas and dust}

"Sometimes you can't stick your head in the engine, so you have to examine the exhaust" Donald E. Osterbrock

Gas and dust, in various states, are important constituents of HzRGs and must play a fundamental role in their evolution. Gas has been observed over a wide range of temperatures and having a variety of forms.

\subsection{Hot ionised gas—radio depolarisation}

X-ray observations show that hot ionised gas is widespread at the centres of clusters and around radio galaxies at low redshifts. Typical derived parameters are temperatures of $\sim 10^{7.5} \mathrm{~K}$, densities of $0.05 \mathrm{~cm}^{-2}$, and masses of a few $\times 10^{12} \mathrm{~cm}^{-3}$, with pressures of $\sim 10^{-9}$ dyne $\mathrm{cm}^{-2}$. Such a gas would be sufficient to confine both the radio-emitting plasma and the Ly $\alpha$ halos (see Sect. 3.2) of HzRGs. For low and intermediate redshift clusters, bubble-like structures in X-rays have been attributed to energy injection into such a hot intracluster medium by radio jets (e.g. McNamara et al. 2000).

The sensitivity of the present generation of X-ray telescopes is only marginally sufficient for detecting similar hot gas at $z \sim 2$. X-ray emission has been observed from the Spiderweb Galaxy at $z=2.2$ (Fig. 4) (Carilli et al. 2002b). The X-ray emission is 


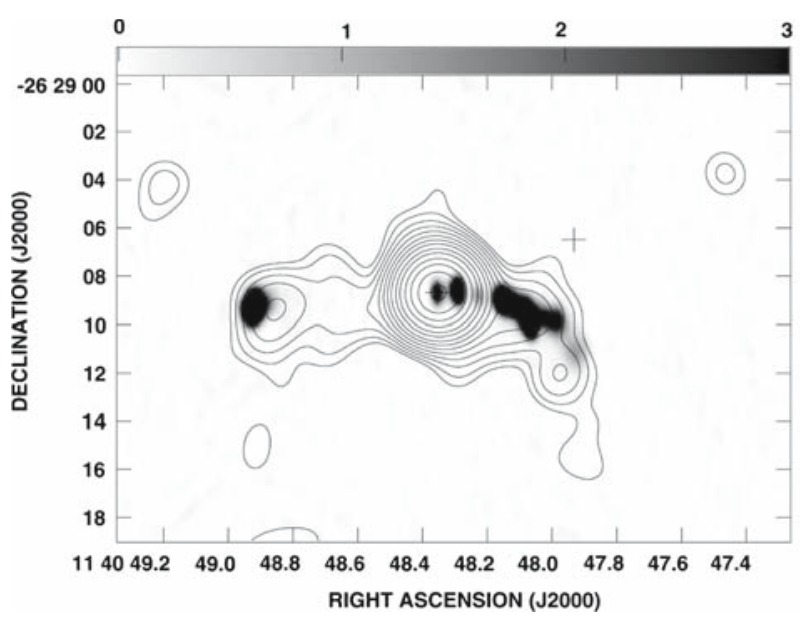

Fig. 4 X-ray emission from the Spiderweb Galaxy, PKS 1138-262 at $z=2.2$, observed with the Chandra X-ray Telescope [from Carilli et al. (2002b)]. X-ray contours are superimposed on a VLA gray scale representation of the $5 \mathrm{GHz}$ radio continuum emission at $0.5^{\prime \prime}$ in resolution. The cross marks the position of the radio galaxy nucleus. Note that the X-ray and radio emission are aligned with each other. See also Fig. 16.

extended along the radio source axis and attributed to thermal emission produced by shocks as the synchrotron-emitting relativistic jet that propagates outwards.

An alternative technique for detecting and studying this hot gas is through its effect on the polarisation of background radio emission (e.g. Miley 1980). As it propagates through a magnetoionic medium the polarisation of linearly polarised radiation is rotated through an angle $\chi$ that is proportional to the square of the wavelength, $\lambda(\mathrm{cm})$.

$\Delta \chi=5.73 \times 10^{-3} \mathrm{RM} \lambda^{2}$, where $R$ is the observed rotation measure, given by $\mathrm{RM}$ is $\mathrm{RM}=812 \int n_{t}(s) B_{\|}(s) \mathrm{d} s \sim 8.1 \times 10^{8} n_{t} B_{\|} s\left(\mathrm{rad} \mathrm{m}^{-2}\right)$.

Here $s(\mathrm{kpc})$ is the path length through the medium, $n_{t}\left(\mathrm{~cm}^{-3}\right)$ is the density of thermal electrons and $B_{\|}$is the component of magnetic field along the line of sight. The intrinsic rotation measure is $\mathrm{RM}=\mathrm{RM}_{o b s} \times(1+z)^{2}$.

Observations of radio polarisation data for $>40 \mathrm{HzRGs}$ (Carilli et al. 1994, 1997; Athreya et al. 1998b; Pentericci et al. 2000b; Broderick et al. 2007) revealed that several sources have extremely large rotation measures (RMs), up to more than $1,000 \mathrm{rad} \mathrm{m}^{-2}$ in the rest frame (Carilli et al. 1997; Athreya et al. 1998b) (e.g. Fig. 5), with considerable variation across the sources. An obvious interpretation of the large observed rotation measures is that HzRGs are embedded in dense gas. At low-redshifts the largest rotation measures are observed for radio sources that are located in X-ray emitting clusters that are inferred to be "cooling flows" (Ge and Owen 1994). Hence the large rotation measures of HzRGs are strong circumstantial evidence that these objects are also located in cluster environments at high redshifts (Sect. 7).

Assuming that (i) the rotation is produced over a line-of-sight path length that is comparable with the projected dimensions of the radio sources and (ii) the magnetic field strength is close to the equipartition values, the derived electron densities are $\sim 0.05 \mathrm{~cm}^{-3}$, comparable to those obtained for low-redshift clusters from the X-rays. 


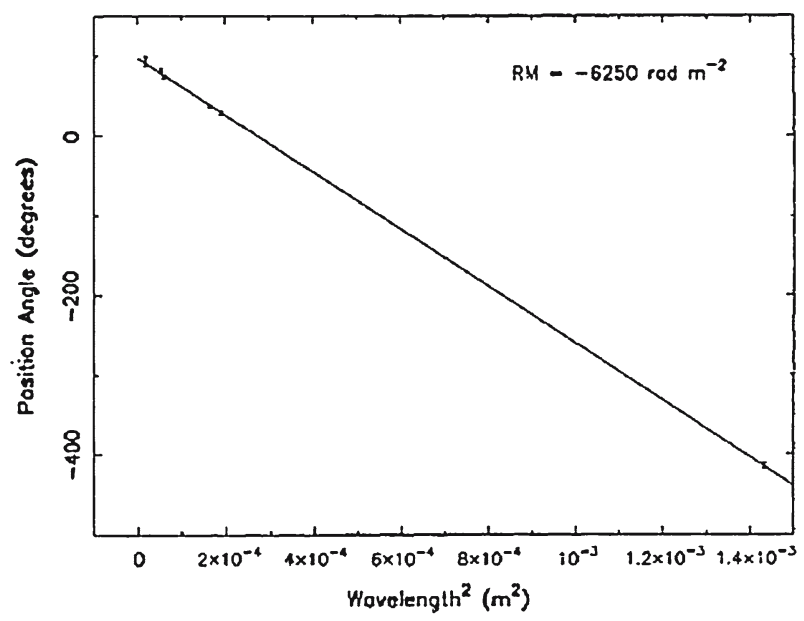

Fig. 5 The observed polarisation position angle as a function of the square of the wavelength ${ }^{2}$ for a radio knot of 4C41.17 at $z=3.8$ [from Carilli et al. (1994)]. The knot is the brightest of the 4 radio knots shown in Fig. 2. Note the excellent fit to a $\lambda^{2}$ dependance and the large resultant rotation measure for the knot

\subsection{Warm ionised gas}

The bright emission lines emitted by luminous radio galaxies allow their redshifts to be determined and have been an important reason why radio galaxies have been such important cosmological probes. The emission lines are emitted by warm $\left(10^{4.5} \mathrm{~K}\right)$ gas and provide a powerful tool for studying physical conditions within the HzRGs.

Emission lines observed from active galactic nuclei are both "permitted" recombination lines and collisionally excited "forbidden" lines. When a free electron is captured by an atomic nucleus it cascades to the ground state emitting a series of recombination lines. The most prominent recombination lines are due to the most common elements, hydrogen and helium. However, some of the strongest lines in the spectra of emission nebulae correspond to so-called "forbidden" transitions that have miniscule probability of occurring relative to the permitted transitions, but whose energies lie within a few $\mathrm{kT}$ of the ground levels and can therefore be easily populated by collisions. Each element has a critical density below which most de-excitations are radiative and a line is produced. Forbidden lines are only produced by gas with densities in the range $\sim 10-10^{5} \mathrm{~cm}^{-3}$ and the line ratios contain important information about the density, temperature, ionisation and abundances of the emitting gas (Osterbrock and Ferland 2006; Peterson 1997).

A composite emission-line spectrum for HzRGs, and a list of emission line strengths were given by McCarthy (1993). During the early nineties it was found that, for high-redshift radio galaxies, nuclear emission lines are generally accompanied by an additional component that is highly extended spatially. The presence of giant luminous ionised gas nebulae are amongst the most remarkable features of HzRGs (e.g. Reuland et al. 2003a). These "halos" have sizes of up to $200 \mathrm{kpc}$ (e.g. Fig. 16) and their study provides a wealth of information about kinematics and physical conditions within and surrounding the HzRGs and on the origin of the gas. 


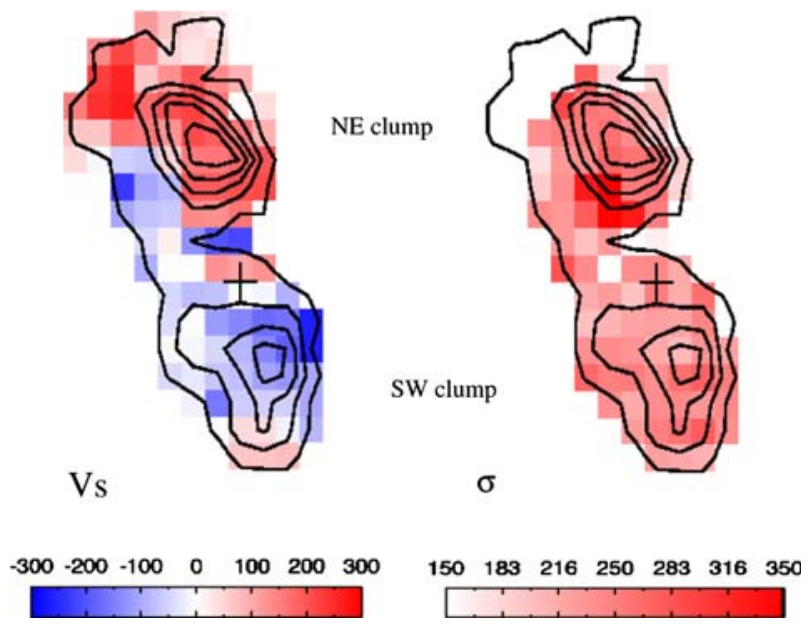

Fig. 6 Velocities and velocity dispersions across MRC 2104-242 at $z=2.5$ measured with VIMOS on the VLT [from Villar-Martín (2007)]. This HzRG is surrounded by a giant Lya nebula that extends by $\sim 120 \mathrm{kpc}$ The position of the radio core is indicated with a cross. The velocity field appears symmetric and ordered implying either rotation or radial motions. See also van Ojik et al. (1996)

During the last 15 years considerable progress has been made in studying the properties of Ly $\alpha$ halos, using large ground-based optical/IR telescopes. Observations of their emission line spectra have been extended into the infrared (optical rest-frame), where additional useful physical diagnostics can be obtained. Also, the kinematics of several HzRGs have been mapped in detail. An excellent recent review of the emission-line properties of HzRGs has been given by Villar-Martín (2007). For detailed information the reader is referred to that review and the references therein.

The ionised gas halos have Ly $\alpha$ luminosities of typically $\sim 10^{43.5} \mathrm{erg} \mathrm{s}^{-1}$ and line widths varying from a few hundred $\mathrm{km} / \mathrm{s}$ in their outer parts to $>1000 \mathrm{~km} \mathrm{~s}^{-1}$, near the galaxy nuclei. The usual emission-line diagnostics (Osterbrock and Ferland 2006) show that the gas has temperatures of $T_{e} \sim 10^{4}-10^{5} \mathrm{~K}$, densities of $n_{e} \sim 10^{0.5}-10^{1.5} \mathrm{~cm}^{-3}$ and masses of $\sim 10^{9}-10^{10} \mathrm{M}_{\odot}$. The warm gas occupies a relatively small fraction of the total volume of the HzRGs, with filling factors estimated to be $\sim 10^{-5}$ compared with unity for the hot gas. Although the topology of the gas is not well determined, the properties of the emission lines and the covering factor deduced from statistics of absorption lines (Sect. 3.3.2) clouds led to a model (van Ojik et al. 1997a) in which the halos are composed of $\sim 10^{12}$ clouds, each having a size of about 40 light days, i.e. comparable with that of the solar system. van Ojik et al. (1997a) speculate that the clouds might be associated with the early formation stages of individual stars.

The morphologies of the Ly $\alpha$ halos are clumpy and irregular. Their overall structures are often aligned with the radio axes and sometimes extend beyond the extremities of the radio source. There appear to be two distinct regimes in the halos that often blend together. The inner regions close to the radio jets are clumpy, with velocity spreads of $>1,000 \mathrm{~km} \mathrm{~s}^{-1}$. They appear to have been perturbed by the jets. The outer regions are more quiescent, with velocity ranges of a few hundred $\mathrm{km} \mathrm{s}^{-1}$ ). They appear to be more relaxed than the inner regions (e.g. Fig. 7). 

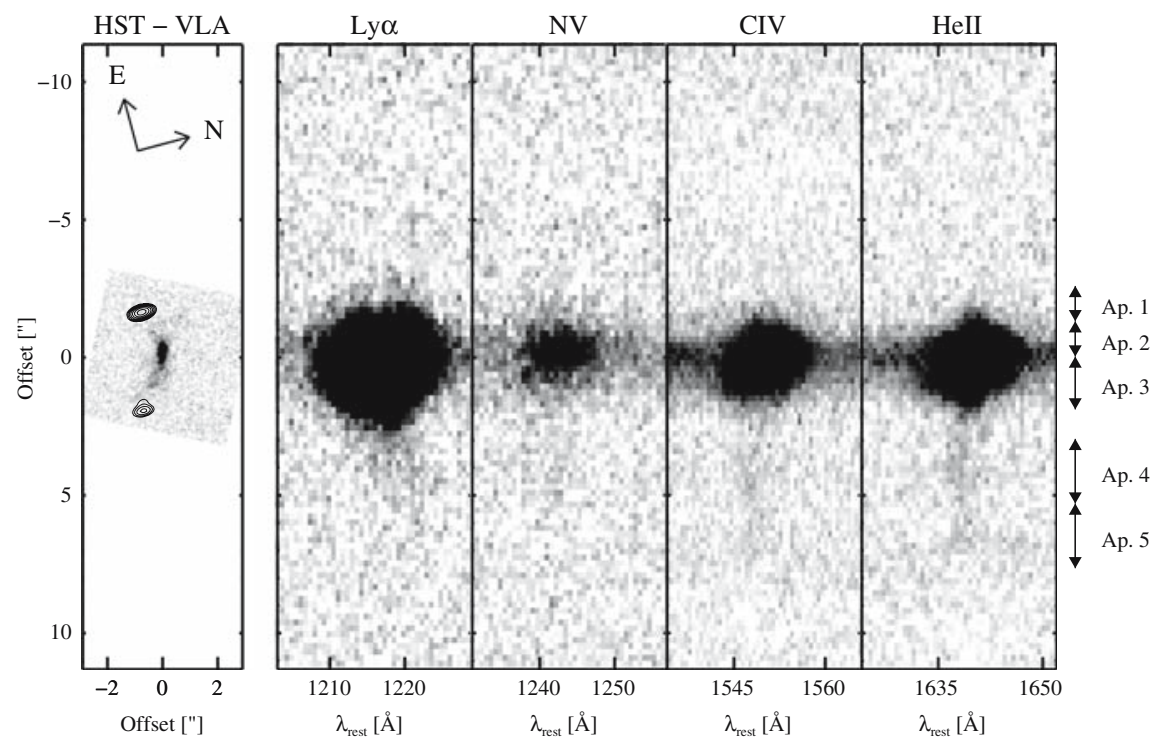

Fig. 7 Long slit spectroscopy of the ionised gas halo in MRC 0943-242 at $z=2.9$ [from Villar-Martín (2007); Humphrey et al. (2006)]. VLA radio map overlaid with the WFPC2 HST image (left), spatially aligned with the 2-dimensional Keck spectra of the main UV rest frame emission lines. The impact of jet-gas interactions on the observed properties of the giant nebula (size $\sim 70 \mathrm{kpc}$ ) can be seen in the much broader and brighter emission lines within the radio structures compared with the faint emission detected beyond

The relative intensities of emission lines are, in principle, powerful diagnostic tools for studying physical conditions in the warm line-emitting gas. However, disentangling the effects of ionisation, abundances, density and temperature using the emission line ratios is complicated and requires detailed modelling, incorporating all facets of the HzRGs. We shall now discuss the physical conditions within the warm gas halos in more detail.

\subsubsection{Ionisation}

Various mechanisms have been proposed for exciting the gas. These include (i) photoionisation from an AGN, (ii) photoionisation from stars, (iii) photoionisation by ionizing X-rays emitted by shocked hot gas and (iv) collisional ionisation from shocks. Plots of optical-line ratios have been used extensively to study the ionisation of gas in nearby active galaxies, where evidence for both jet- and accretion-powered shocks and for photoionisation by the central AGN has been found (e.g. Villar-Martín and Binette 1997; Villar-Martín et al. 1997; Bicknell et al. 2000; Groves et al. 2004a,b).

Although these relationships have been most accurately calibrated in the rest-frame optical region of the spectrum, line diagnostic diagrams have also been developed for use in the ultraviolet (Allen et al. 1998; Groves et al. 2004b), the spectral region of HzRGs sampled by optical observations. Interpretation of emission line ratios are complicated by effects of dust and viewing angles (Villar-Martín and Binette 1996). 
Also most HzRG spectra are spatially integrated over regions of 10 or more kiloparsec, where conditions and sources of excitation may change. In a recent comprehensive study using as many as 35 emission lines throughout the rest-frame UV and optical spectra Humphrey et al. (2006) concluded that photoionisation is the dominant source of excitation in the quiescent gas. A harder source of photoionisation than stars is needed, consistent with photons from an AGN.

If a quasar is exciting the warm gas in the halo, why do we not see it? The usual explanation is that the quasar emits radiation anisotropically. It is highly absorbed in the direction in which we view it, but not along the radio axis. Support for this idea is provided by the large optical polarisations measured for some HzRGs. (Sect. 3.5.2). However, if highly anisotropic flux from a hidden quasar is indeed the dominant source of excitation, it is difficult to understand why many of the Ly $\alpha$ halos are approximately symmetric in shape. An alternative explanation is that the quasar activity is isotropic and highly variable, with short sharp periods of intense activity and longer periods of relative passivity, when the quasar is dormant (see Sect. 5.1).

Although photoionisation by a quasar is presently the "best bet" for the dominant source of excitation, it is not likely to be the only culprit. Considerable variation is observed in the relative emission line strengths from object to object and within individual HzRGs. There is strong evidence that there is also collisional excitation from shocks, particularly close to the radio jets-(see e.g. Bicknell et al. 2000; Best et al. 2000; Sect. 4.3).

\subsubsection{Abundances and star formation}

Although Ly $\alpha$ is by far the brightest emission line emitted by the warm gas halos, other lines are also detected. In 4C41.17 at $z=3.8$, kinematical structures in the Ly $\alpha$ line are closely followed in the carbon CIV and oxygen [OII] and [OIII] lines, with the $[\mathrm{OIII}] \lambda 5007$ emission extending by as much as $60 \mathrm{kpc}$ from the nucleus (Reuland et al. 2007). See also Fig. 7.

The chemical abundance of the halo gas is close to solar (Vernet et al. 2001b; Humphrey et al. 2006), consistent with the HzRG having undergone prodigious star formation at earlier epochs. Further indications that the star formation rate in HzRGs was higher in the past come from measurements of the Ly $\alpha$ luminosities and Ly $\alpha / \mathrm{HeII}$ ratios that are both systematically larger for HzRGs with $z>3$, than for those with $2<z<3$ (Villar-Martín et al. 2007a,b).

The relative intensity of the NV $\lambda 1240 \AA$ line varies from being an order of magnitude fainter than the carbon and helium lines (De Breuck et al. 2000a) to being as luminous as $\operatorname{Ly} \alpha$ (e.g. van Ojik et al. 1994). This has been interpreted as indicating that there are large variations in metallicity between HzRGs.

\subsubsection{Inner kinematics: outflows, jet interactions and superwinds}

In considering the kinematics of the ionised gas halos, we shall separately consider the nuclear turbulent regions, where there is evidence for outflows and the outer passive regions where the most likely dominant systematic motions are inwards. The inner regions show shocked gas closely associated with the radio lobes. These dis- 
play disturbed kinematics and have expansion velocities and/or velocity dispersions $>1,000 \mathrm{~km} \mathrm{~s}^{-1}$.

Besides synchrotron jets there is evidence that starburst "superwinds" (Armus et al. 1990; Zirm et al. 2005) are also present in the inner regions of the Ly $\alpha$ halos. Both the jets and the superwinds will exert sufficient pressure on the warm gas to drive it outwards. Interactions between radio jets and the ambient gas is important in some low-redshift radio galaxies (e.g. Heckman et al. 1982, 1984; van Breugel et al. 1984, 1985a,b). The radio sources are observed to excite, disturb and entrain the gas. Likewise, the gas can bend and decollimate radio jets and enhance the intensity of their radio emission through shock-driven particle acceleration. In general small radio sources show more jet-gas interactions than large ones (Best et al. 2000). At $z>2$, HzRG exhibit signatures of even more vigorous jet-gas interactions (e.g. Villar-Martín et al. 1998, 1999a,b, 2003; Humphrey et al. 2006). The kinematics is more turbulent and the ionisation is higher in the region of the jet than in the quiescent outer halo (Humphrey et al. 2006).

Nesvadba et al. (2006) recently carried out an important study of the Spiderweb Galaxy with an integral field spectrograph in the optical rest frame. They make a convincing case that there is an accelerated outflow of warm gas in this object. The only plausible source of energy for powering this outflow (few $\times 10^{60} \mathrm{erg}$ ) is the radio jet and, even then, the coupling between the jet and the ISM must be very efficient to account for the observed kinematics. The pressure in the radio jets can drive gas outwards from the nuclei for tens of kiloparsecs and play an important role, together with starburst-driven superwinds, in "polluting" the intergalactic medium with metals. There is spectroscopic evidence for the ejection of enriched material in 4C 41.17 at $z=3.8$ up to a distance of $60 \mathrm{kpc}$ along the radio axis (Reuland et al. 2007). See also Fig. 7.

In Sect. 4.3 we shall discuss strong evidence that the jet-gas interactions can also trigger star formation in HzRGs (Fig. 6).

\subsubsection{Outer kinematics: infall of the quiescent halos}

An important diagnostic in tracing the origin of the warm gas is the kinematics in the outer region of the giant halos, where they are apparently unperturbed by the radio jet. The outer halos displays systematic velocity variations of a few hundred $\mathrm{km} / \mathrm{s}$ (e.g. see Fig. 7). Are these systematic velocity variations the result of rotation (van Ojik et al. 1996; Villar-Martín et al. 2003, 2006b), outflows (Zirm et al. 2005; Nesvadba et al. 2006) or infalling motions?

It is difficult to discriminate between the various kinematic scenarios from velocity data alone, but comparison of spectroscopic and radio data provides additional relevant information. In a study of $11 \mathrm{HzRGs}$ with redshifts $2.3<z<3$.6, several correlated asymmetries were found between the halo kinematics and assymetries in the radio structures (Humphrey et al. 2007; Villar-Martín et al. 2007b). On the side of the brightest radio jet and hot spot, the quiescent nebula appears systematically redshifted (receding) compared with the other side. On the assumption that the bright radio jet and hot spot are moving towards us and brightened by Doppler boosting (Rees 
1967; Kellermann 2003), the quiescent gas must be moving inwards. The brightest radio hotspot is also the least depolarised one, as expected if it is on the closest side of the HzRG and consistent with the infall scenario.

Could the inflowing motion of the gas be a result of cooling flows, that have long been studied in clusters of galaxies at low redshifts (e.g. Fabian 1994; Kaufmann et al. 2006)? Recent XMM and Chandra observations have shown that the cooling rates are reduced by an order of magnitude below the simple cooling flow models at temperatures $<\times 10^{7} \mathrm{~K}$, probably through interaction of the gas with radio sources associated with galaxies in the cluster. An alternative diagnostic of cooling flows that are more feasible for HzRGs than X-rays is the measurement of roto-vibrational lines from $\mathrm{H}_{2}$ molecules at $\sim 2,000 \mathrm{~K}$ (Jaffe and Bremer 1997; Jaffe et al. 2005). At high redshifts these lines are shifted from the near to mid-IR bands, accessible with the Spitzer Telescope. The $\mathrm{H}_{2}$ lines are a promising tool for studying both the cooling in the gas around HzRGs and the excitation mechanisms.

To summarise, the kinematics of the warm gas is complex. There is evidence that gas in the outer regions of the halo is flowing inwards, providing a source of material for feeding the active nucleus (Sect. 5) and that gas in the inner region is being driven outwards by pressure from jets and starbursts. The various motions in the ionised gas halos are likely to contribute to feedback processes between the AGNs and the galaxies invoked in current models of massive galaxy evolution (Sect. 6).

We end our discussion of the halo kinematics on a cautionary note. Most kinematic studies of HzRGs until now have been based on Ly $\alpha$, because of its relatively large equivalent width and its accessibility with optical telescopes. However, Ly $\alpha$ is a resonant line and subject to strong scattering and optical depth effects. Hence, the resultant kinematics may not be completely representative of the gas as a whole, particularly in the inner regions.

\subsubsection{Relation to non-radio Lya nebulae}

There may well be a connection between Ly $\alpha$ halos in HzRGs and the disembodied extended Ly $\alpha$ nebulae that have been discovered in recent years (e.g. Fynbo et al. 1999; Steidel et al. 2000; Francis et al. 2001; Matsuda et al. 2004; Dey et al. 2005; Colbert et al. 2006; Nilsson et al. 2006). These nebulae also have physical extents $\gtrsim 100 \mathrm{kpc}$ and Ly $\alpha$ line fluxes of $\sim 10^{-15} \mathrm{ergs} \mathrm{s}^{-1} \mathrm{~cm}^{-2}$. Although in many respects, these Ly $\alpha$ blobs resemble the giant ionised gas halos around HzRGs, they have $<1 \%$ of the associated radio continuum flux and no obvious source of UV photons bright enough to excite the nebular emission. However, millimetre emission has been detected from several of these nebulae (Chapman et al. 2001; Smail et al. 2003; Geach et al. 2005) and Matsuda et al. (2004) suggest that the extended Ly $\alpha$ nebulae are also associated with dense environments in the early Universe. Just like the HzRG halos, these nebulae can be excited by quasars that are heavily obscured along the line of sight (Haiman and Rees 2001; Weidinger et al. 2004, 2005), or quasars that undergo recurrant flares (Sect. 5.1). Alternatively, they may be associated with cooling-flow-like phenomena (Haiman et al. 2000; Dijkstra et al. 2006a,b). 


\subsubsection{Nebular continuum}

Dickson et al. (1995) pointed out that the nebular continuum emission due to the line-emitting warm gas of HzRGs is significant and must be taken into account when computing the various contributions to the UV continuum. See also (Aller 1984; Vernet et al. 2001b; Humphrey et al. 2007). However, in general the nebular component contributes $<25 \%$ of the continuum emission at $1,500 \AA$, and is much less important than starlight or emission from a hidden or dormant quasar. The contibution from nebular continuum can be quite accurately predicted by means of the strength of the HeII $\lambda 4686 \AA$ line, or when not available the HeII $\lambda 1640 \AA$ line (Aller 1984).

\subsection{Neutral gas}

There are two techniques for studying cool HI gas in HzRGs. One method is to measure redshifted absorption of the $21 \mathrm{~cm}$ hydrogen line against the bright radio continuum. The second method is to observe deep narrow absorption troughs that are often present in the Ly $\alpha$ profiles. In principle, both these techniques can be used to constrain properties of the neutral hydrogen such as spatial scales, mass, filling factor, spin temperature and kinematics (Röttgering et al. 1999a).

\subsubsection{HI absorption}

Neutral hydrogen (HI) atoms are abundant and ubiquitous in low-density regions of the ISM. They are detectable by means of the hyperfine transition, emitting at $1420.405751 \mathrm{MHz}(\sim 21 \mathrm{~cm})$. Observations of atomic neutral hydrogen by means of this line has been one of the most powerful tools of radio astronomy since its inception. Studying the $21 \mathrm{~cm}$ HI line in absorption has been an important probe of HI around low-redshift radio galaxies (see the recent review of Morganti (2006)). Limitations of such searches for HI in high-redshift radio galaxies include the availability of lownoise receivers that cover the observing frequencies dictated by the target redshifts and problems due to radio frequency interference. In $1991 \mathrm{HI}$ absorption was detected in the HzRG, B2 $0902+34$ at $z=3.4$, by Uson et al. (1991). See Fig. 8. Since then progress in this field has been disappointing. For a review see Röttgering et al. (1999a). Besides some follow-up work on B2 0902 + 34 (Briggs et al. 1993; De Bruyn 1996; Cody and Braun 2003; Chandra et al. 2004), there have been only two tentative but unconfirmed additional detections of $\mathrm{HI}$ absorption in B3 $0731+438$ at $z=2.4$ and MG $1019+053$ at $z=2.8$.

The HI absorption line provides a measure of the average column density of the absorbing material weighted by the flux density of the background source. The total column density is $\sim 4.4 \times 10^{18} T_{S}$. Assuming a spin temperature of $T_{S} \sim 10^{3} \mathrm{~K}$, De Bruyn (1996) derives an HI column density of $\sim 2 \times 10^{21} \mathrm{~cm}^{-2}$ for B2 $0902+34$ and a corresponding mass of neutral hydrogen of $M_{H I} \sim 3 \times 10^{7} M_{\odot}$.

Why has HI absorption not been detected in a larger number of HzRGs, despite extensive searches? A possible explanation is that the HI absorption is caused by small $\sim 100$ pc-sized disks or torus-like structures, aligned perpendicular to the radio source 

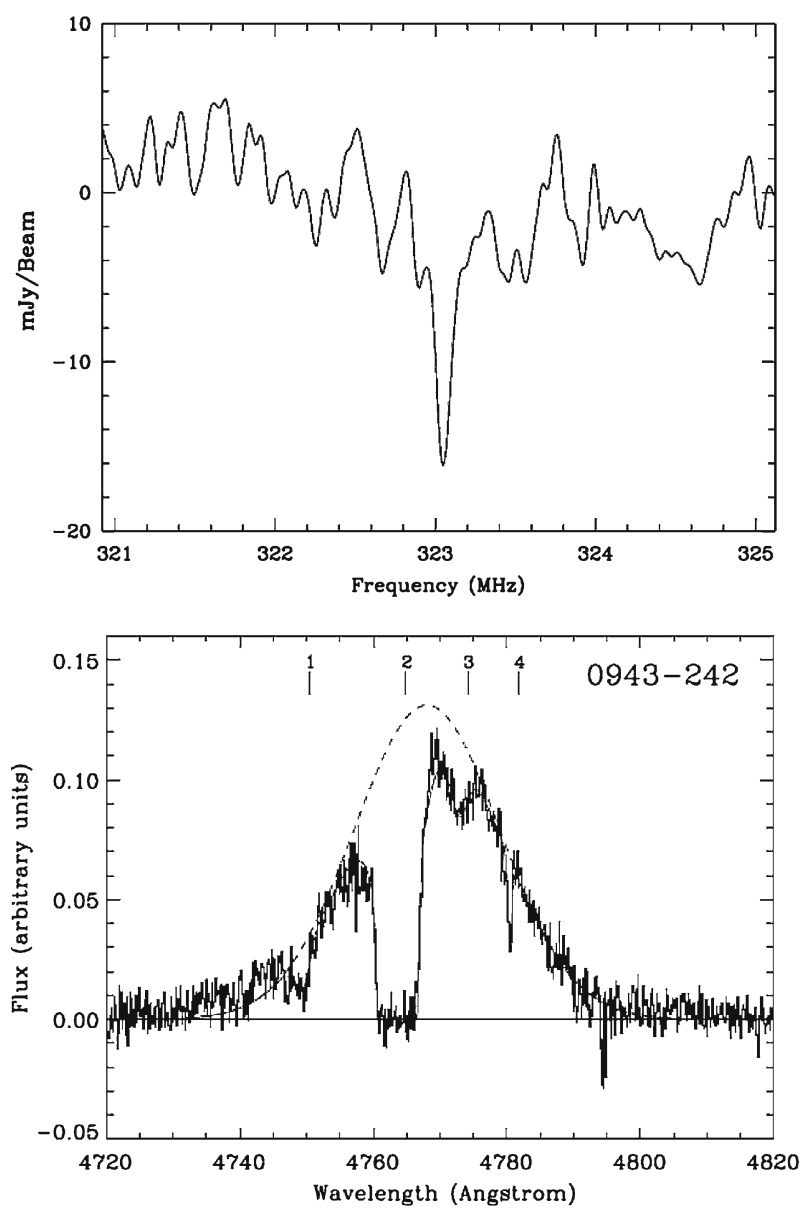

Fig. 8 Absorbing neutral hydrogen. Top The redshifted HI absorption profile of $0902+34$ at $z=2.4$ [from Cody and Braun (2003)]. Bottom The redshifted Ly $\alpha$ absorption profile of 0943242 at $z=2.9$, with a model overlaid [from Jarvis et al. (2003)]

(Röttgering et al. 1999a). The radio emission of $0902+34$ is more centrally concentrated than that for most HzRGs, consistent with the hypothesis that the absorption is produced by such a small disk. For the more extended radio sources associated with most HzRGs, the disk covering fraction would be very small and the disk would not produce significant absorption.

\subsubsection{Lya absorption}

More than a decade ago van Ojik et al. (1997a) discovered that strong absorption features are common in the Ly $\alpha$ profiles of HzRGs. Such features were present in the majority of the $18 \mathrm{HzRG}$ that they studied with sufficient spectral resolution. Derived column densities were in the range $10^{18}-10^{19.5} \mathrm{~cm}^{-2}$. 
The absorption, usually interpreted as being due to HI surrounding the HzRG, provides an interesting diagnostic tool for studying and spatially resolving neutral gas surrounding HzRGs. Because the spatial extension of the absorbing region can be constrained, the Ly $\alpha$ absorption lines provide information about properties of the absorbing gas (e.g. dynamics and morphologies) that cannot be studied using quasar absorption lines. Since in most cases the Ly $\alpha$ emission is absorbed over the entire spatial extent (up to $50 \mathrm{kpc}$ ), the absorbers must have a covering fraction close to unity. From the column densities and spatial scales of the absorbing clouds, the derived masses of neutral hydrogen are typically $\sim 10^{8} \mathrm{M}_{\odot}$.

Additional information about the properties of the HI absorbers was obtained by Wilman et al. (2004). In a study of $7 \mathrm{HzRG}$ with $2.5<z<4$. 1, they identified two distinct groups of $\mathrm{HI}$ absorbers: strong absorbers with column densities of $N_{\mathrm{HI}} \sim 10^{18}$ to $10^{20} \mathrm{~cm}^{-2}$ and weaker systems with $N_{H I} \sim 10^{13}$ to $10^{15} \mathrm{~cm}^{-2}$. They suggest that the strong absorbers may be due to material cooling behind the expanding bow shock of the radio jet and that the weak absorbers form part of the multiphase protointracluster medium responsible for the Ly $\alpha$ forest. Furhermore, Krause (2005) carried out hydrodynamic simulations of a HzRG jet inside a galactic wind shell and showed that strong HI absorption could be produced.

Absorption is occasionally observed in the profiles of other emission lines than Ly $\alpha$, such as CIV_-(Röttgering et al. 1995). Jarvis et al. (2003) studied the profiles of two of the most prominent absorbing HzRGs MRC $0943-242$ at $z=2.9$ and TXS $0200+015$ at $z=2.2$ with high spectral resolution. The data are consistent with a picture in which the absorbing gas has low density and low metallicity and is distributed in a smooth absorbing shell located beyond the emission-line gas. However, the metallicity, inferred from the C IV absorption, is considerably lower in $0943-242$ than in the slightly larger source TXS $0200+015$. This difference in metallicity is explained as due to chemical enrichment via a starburst-driven superwind (Sect. 3.2.3). Further observations and modelling of the spectrum of MRC $0943-242$ by Binette et al. (2000) indicate that in this object the absorbing gas may actually be ionised-see also (Binette et al. 2006). However, MRC $0943-242$ may be a special case. It has a relatively small radio size and one of the deepest $\operatorname{Ly} \alpha$ absorption trough of all known absorbers.

\subsection{Molecular gas}

Star formation is generally observed to occur in molecular clouds-cold dark condensations of molecular gas and dust, that are observable in the millimetre and near-IR. In these clouds atomic hydrogen associates into molecular hydrogen, $\mathrm{H}_{2}$, a species that unfortunately does not emit easily observable spectral lines. The next most abundant molecule is carbon monoxide. Because the rotational transitions of the dipolar molecule ${ }^{12} \mathrm{CO}$ are caused primarily by collisions with $\mathrm{H}_{2}, \mathrm{CO}$ is an excellent tracer of molecular hydrogen. The most important redshifted $\mathrm{CO}$ transitions for the study of high-redshift objects are $J=(1-0),(2-1),(3-2),(4-3)$ and (5-4) at 115.2712, 230.5380, 345.7960, 461.0407 and $576.2677 \mathrm{GHz}$, respectively. These lines are an important diagnostic for probing the reservoir of cold gas available for star formation. 
Intensive searches for CO emission from HzRGs during the early 1990s were unsuccessful (Evans et al. 1996; van Ojik et al. 1997b). Since then, the sensitivity of (sub)millimetre receivers has been improved and the high-redshift Universe has been opened to molecular line studies (see reviews by Solomon and vanden Bout (2005) and Omont (2007)). Table 2 is a list of HzRGs detected in CO.

To convert the $\mathrm{CO}$ to $\mathrm{H}_{2}$ mass, one often assumes a standard conversion factor. For high-redshift CO studies, this factor is calibrated based on observations of nearby ultra-luminous infrared galaxies (ULIRGS) (Downes et al. 1993). With the assumption that this value is also applicable to high-redshift objects, one can use the strength of the (1-0) CO transition to derive the mass of the molecular gas. Because current centimetre telescopes do not allow observations of the (1-0) transition at $z<3.6$, one needs to observe higher order transitions shifted to the atmospheric windows at 3,2 and $1.3 \mathrm{~mm}$.

The inferred masses of $\mathrm{H}_{2}$ in the CO-detected galaxies are between $10^{10}$ and $10^{11} \mathrm{M}_{\odot}$, indicating that there is a large mass of molecular gas in these objects and a substantial reservoir of material available for future star formation. However, the calculated masses should be treated with caution, because their derivation is based on a large number of assumptions (e.g. Downes et al. 1993). Observations of higher CO transitions are biased to the detection of denser gas than ground-state observations and can result in an underestimate of the total molecular gas mass.

Observations of multiple rotational transitions allow the temperature and density of the molecular gas to be constrained using large velocity gradient models. The results indicate that the CO properties are heterogeneous. TN J0924-2201 is only detected in the ground-state (1-0) transition, but $4 \mathrm{C} 41.17$ is only detected in $\mathrm{CO}(4-3)$, despite sensitive searches for the (1-0) transition (Ivison et al. 1996; Papadopoulos et al. 2005). This high excitation level in 4C41.17 implies large gas densities $n\left(\mathrm{H}_{2}\right)>1,000 \mathrm{~cm}^{-3}$, consistent with gas fueling a nuclear starburst.

Table 2 HzRGs detected in $\mathrm{CO}$

\begin{tabular}{lllllll}
\hline Name & $z$ & Transition & $\begin{array}{l}\Delta V_{\mathrm{CO}} \\
\left(\mathrm{km} \mathrm{s}^{-1}\right)\end{array}$ & $\begin{array}{l}S_{\mathrm{CO}} \Delta V \\
\left(\mathrm{Jy} \mathrm{km} \mathrm{s}^{-1}\right)\end{array}$ & $\begin{array}{l}M\left(\mathrm{H}_{2}\right) \\
\left(10^{10} M_{\odot}\right)\end{array}$ & References $^{\mathrm{b}}$ \\
\hline 53W002 & 2.390 & $(3-2)$ & 420 & $1.2 \pm 0.2$ & 1.2 & 1,2 \\
B3 J2330+3927 & 3.086 & $(4-3)$ & 500 & $1.3 \pm 0.3$ & 7 & 3 \\
TN J0121+1320 & 3.517 & $(4-3)$ & 700 & $1.2 \pm 0.4$ & 3 & 4 \\
6C 1909+72 & 3.537 & $(4-3)$ & 530 & $1.6 \pm 0.3$ & 4.5 & 5 \\
4C 60.06 & 3.791 & $(1-0)$ & $\ldots$ & $0.15 \pm 0.03^{\mathrm{a}}$ & 13 & 6 \\
& & $(4-3)$ & $>1000$ & $2.5 \pm 0.4$ & 8 & 5 \\
4C 41.17 & 3.792 & $(1-0)$ & $\ldots$ & $<0.1$ & $\ldots$ & 7 \\
TN J0924-2201 & 5.197 & $(4-3)$ & 1000 & $1.8 \pm 0.2$ & 5.4 & 8 \\
& & $(5-4)$ & 250 & $1.2 \pm 0.3$ & & 9 \\
\hline
\end{tabular}

\footnotetext{
a Only broad component; narrow component has $S_{\mathrm{CO}} \Delta V=0.09 \pm 0.01 \mathrm{Jy} \mathrm{km} \mathrm{s}^{-1}$

b References: 1 = Scoville et al. (1997), 2 = Alloin et al. (2000), 3 = De Breuck et al. (2003a), 4 = De Breuck et al. (2003b), 5 = Papadopoulos et al. (2000), $6=$ Greve et al. (2004), 7 = Papadopoulos et al. (2005), 8 = De Breuck et al. (2005), $9=$ Klamer et al. (2005)
} 


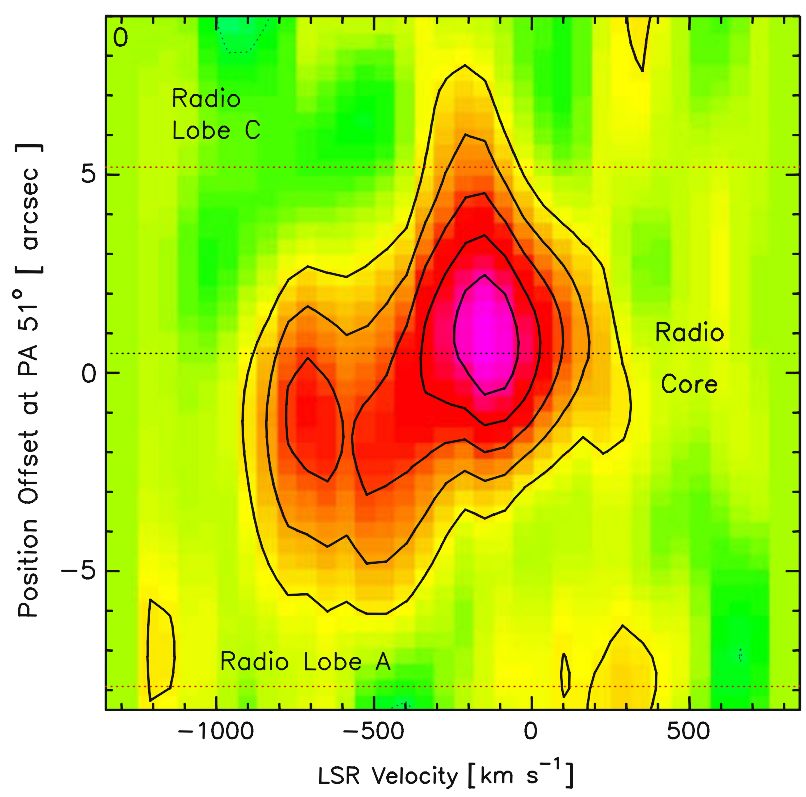

Fig. $9 \mathrm{CO}$ in $4 \mathrm{C} 41.17$ at $z=3.8$ with the Plateau de Bure interferometer [from De Breuck et al. (2005)]. Shown is the velocity plotted against position offset of $\mathrm{CO}(4-3)$, extracted along a $\mathrm{PA}$ of $51^{\circ}$ (see radio morphology in Fig. 2). The central frequency at $96.093 \mathrm{GHz}(z=3.79786)$ is based on the wavelength of the optical HeII $\lambda 1640 \AA$ emission line. The position-velocity diagram shows the two components of the $\mathrm{CO}$ emission. One of these is coincident with the radio core, while a fainter component is spatially offset from it towards the southwestern lobe A

In a few cases the CO appears spatially resolved (Papadopoulos et al. 2000; Greve et al. 2004; De Breuck et al. 2005), extending over 10-20 kpc (e.g. see Fig. 9), providing kinematic information about the molecular gas. Klamer et al. (2004) have claimed that there are alignments between the molecular gas and radio morphologies in some of the detected CO HzRGs, as might be expected from jet-induced star formation (Sect. 4.3). However, higher resolution, larger signal to noise and more statistics are needed before any conclusions about possible alignments can be drawn.

Offsets between the velocities of the molecular gas (CO) and those of the warm gas (e.g. HeII $\lambda 1640$ ) of up to $500 \mathrm{~km} / \mathrm{s}$ have been measured (De Breuck et al. 2003a,b, 2005). Because the $\mathrm{H}_{2}$ masses exceed those of the warm ionised gas Ly $\alpha$ halos by an order of magnitude, the $\mathrm{CO}$ lines provide a better measure of the systemic redshift of the HzRGs than the UV and optical emission lines.

The $\mathrm{CO}$ detections listed in Table 2 are not representative of the distribution of $\mathrm{CO}$ in HzRGs. There are several observational biases inherent in current $\mathrm{CO}$ studies:

- Many searches for $\mathrm{CO}$ at high redshifts pre-select targets on the basis of prior dust detections, i.e continuum millimetre flux (see Sect. 3.5.1). However, $850 \mu \mathrm{m}$ continuum emission was not detected for two of the CO-emitting HzRGs-53W002 at $z=2.390$ (Scoville et al. 1997; Alloin et al. 2000) and TN J0924-2201 at $z=5.2$ (Klamer et al. 2004). This indicates that the gas/dust ratios in high-redshift CO samples could be be systematically underestimated due to bias in favour of dusty targets. 
- Positive detections are more likely to be published than non-detections. Furthermore, the derived limits are often dubious in view of relatively large uncertainties in the targeted redshifts. These uncertainties are due to large offsets that are often present between the $\mathrm{CO}$ and optical redshifts, the unknown widths of the $\mathrm{CO}$ emission lines, and the limited bandwidth of correlators used for the detections.

- For higher redshift objects progressively higher CO transitions need to be observed consistent with the atmospheric transmission bands acessible to millimetre arrays. Hence, measurements of $\mathrm{CO}$ in will tend to trace denser gas in HzRGs than at lower redshifts. However for $z>3.6$, the ground-state transition becomes observable with centimetre facilities, such as the VLA and ATCA, resulting in a discontinuity in the studies of multiple CO transitions needed to constrain the densities and temperatures of the gas.

To investigate the statistics of CO in HzRGs and the evolution of molecular gas, it is important to carry out deep CO observations on well defined samples of HzRGs in multiple $\mathrm{CO}$ transitions, and without a pre-selection on their cold dust properties. The faintness of the $\mathrm{CO}$ lines and the uncertainties in their redshifts means that comprehensive searches for $\mathrm{CO}$ presently require large amounts of observing time. Limiting factors are the sensitivities of current facilities and the relatively limited instantaneous bandwidth covered by the receiver back-ends.

Opportunities for observing molecular gas in HzRGs will be revolutionised during the next decade by ALMA and the EVLA. These facilities will provide an enormous improvement in sensitivity and discovery space throughout the sub-millimetre, millimetre and centimetre wavebands. Furthermore, their resolution will allow the spatial distribution of the molecular gas in HzRGs to be studied. On a shorter timescale, the much wider tuning range and broad-band correlators that are presently coming on-line (e.g. at the EVLA and the ATCA) will provide new opportunities for investigating CO in high-redshift objects, particularly the ground state transition.

The increased sensitivity of ALMA will also open the study of fainter molecular and atomic lines in HzRGs. Lines such as $\mathrm{HCN}$ or $\mathrm{HCO}^{+}$probe at least an order of magnitude denser molecular gas than $\mathrm{CO}$, and are therefore better tracers of the dense molecular cores in star-forming regions (Papadopoulos 2007).

\subsection{Dust}

Dust is an important constituent of HzRGs and an additional diagnostic of star formation. It is both a major constituent of the molecular clouds from which stars generally form and an indicator that substantial star formation has already occurred. The presence of dust means that chemically enriched material is present. Thermal re-radiation from dust often dominates the spectral energy distribution of HzRGs at millimetre and sub-millimetre wavelengths (Fig. 1) and dust is observable as a polarizing and absorbing medium in the optical and ultraviolet.

\subsubsection{Millimetre and sub-millimetre emission}

Since the detection of 4C41.17 at $z=3.8$ (Dunlop et al. 1994; Chini and Kruegel 1994; Ivison 1995) a large number of HzRGs have been observed at millimetre wavelengths 
(Archibald et al. 2001; Reuland et al. 2004). Reuland et al. (2004) analysed a sample of 69 radio galaxies with $1<z<5$, detected at $850 \mathrm{and} /$ or $450 \mu \mathrm{m}$. Isothermal fits to the submillimetre spectra give dust masses of a few $\times 10^{8} \mathrm{M}_{\odot}$ at temperatures of $\sim 50 \mathrm{~K}$ (Archibald et al. 2001).

Possible sources of heating for the dust are X-rays from an AGN (Sect. 5) and UV photons from young stars. The typical submillimetre luminosity (and hence dust mass) of HzRGs strongly increases with redshift, with a $(1+z)^{3}$ dependence out to $z \sim 4$. This is evidence that star formation rates were higher and/or the quasars brighter in HzRGs with $z>3$.

Disentangling whether the dust is heated by an AGN or young stars is difficult, because the two processes produce dust at partially overlapping temperatures. Evidence for heating by young stars comes from the slope of the Rayleigh-Jeans part of the thermal dust emission-observed at millimetre and submillimetre wavelengths. This indicates that the dust temperatures are relatively cool, $\sim 50 \mathrm{~K}$, consistent with starburst-heated emission (e.g. Archibald et al. 2001; Stevens et al. 2003; Reuland et al. 2004). However, there is also evidence for a warmer( $\sim 300 \mathrm{~K}$ dust $)$ component in radio galaxies, consistent with AGN heating (Rocca-Volmerange and Remazeilles 2005). Furthermore, recent Spitzer observations at $5<\lambda_{\text {obs }}<70 \mu \mathrm{m}$ showed that the Wien tail part of the dust SED is inconsistent with low dust temperatures. Dust with temperatures of $\sim 300 \mathrm{~K}$ or higher is required, consistent with AGN heating. Although the submillimetre luminosity of the HzRGs is uncorrelated with radio luminosity, suggesting no strong dependence on the strength of the quasar/AGN emission. (Reuland et al. 2004), the rest-frame $5 \mu \mathrm{m}$ luminosity does appear to be correlated with radio luminosity (Seymour et al. 2007).

It is likely that young stars and AGN both play a role in heating the dust. Clarification of their relative importance await accurate measurement of the SED near the peak of the thermal dust emission by the Herschel Telescope and high spatial resolution observations of the radial profiles of the thermal dust emission with ALMA. Once properly isolated, starburst-heated dust emission will be a powerful tool for measuring the star-formation rates in radio galaxies. On the assumption that most of the submillimetre emission is heated by stars, derived star formation rates are up to a few thousand $M_{\odot} \mathrm{yr}^{-1}$, consistent with those obtained from UV absorption line measurements (Sect. 4.2).

\subsubsection{UV continuum polarisation}

The rest-frame UV polarisation is an important probe of dust in the inner regions of HzRGs (di Serego Alighieri et al. 1989; di Serego Alighieri 1997; Cimatti et al. 1993; Cimatti et al. 1998; Vernet et al. 2001b). The fractional polarisation provides a unique tool for determining the contribution and nature of of scattered light, while the polarisation angle allows the location of the scattering medium to be pinpointed. However, polarisation measurements of HzRGs require both high sensitivity and high precision.

Eight of $10 \mathrm{HzRGs}$ studied by Vernet et al. (2001b), show high continuum polarisation just redward of Ly $\alpha$, with fractions, $f_{p}$ ranging from 6 to $20 \%$ (e.g. Fig. 10). The shape of the polarised flux (= percentage polarisation $\times$ total intensity) is very similar 


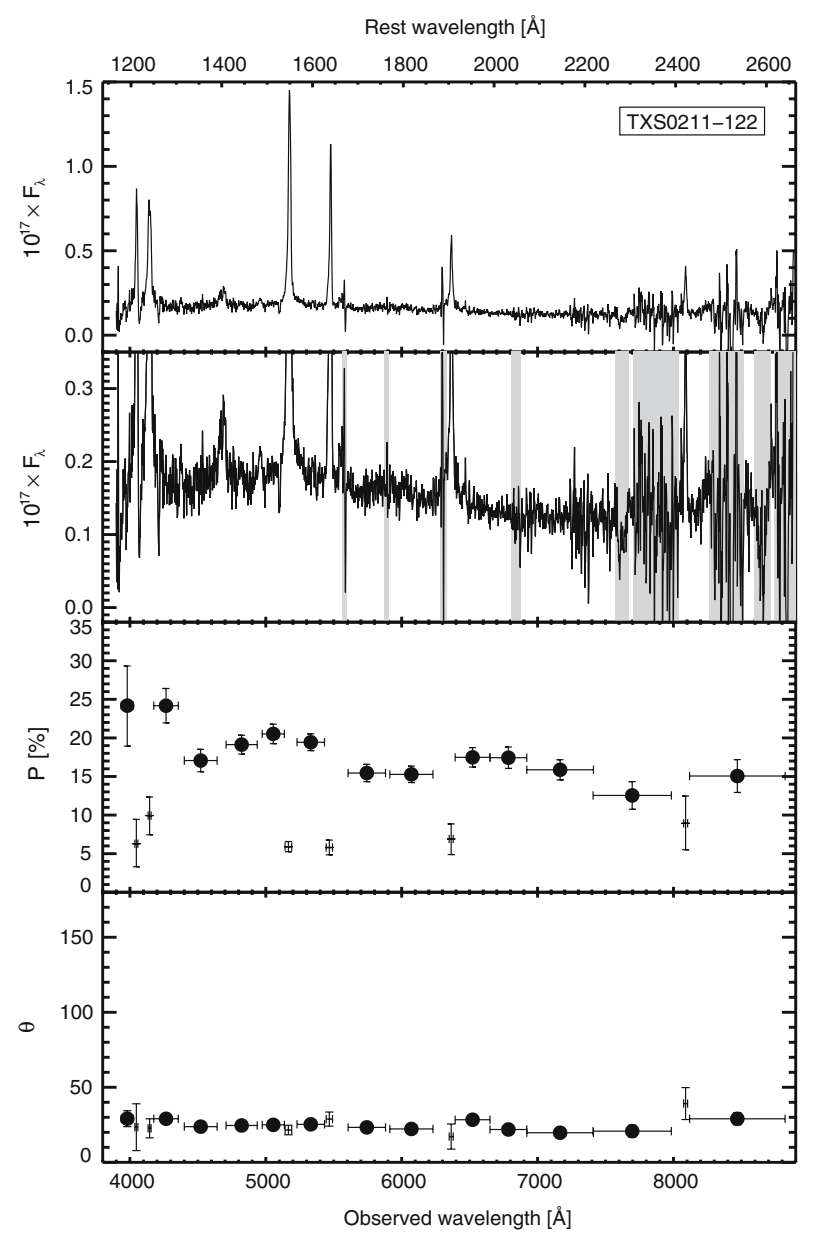

Fig. 10 Spectral and polarisation properties of TXS 0211-122 at $z=2.3$ taken from observations with the Keck Telescope [from Vernet et al. (2001b)]. Plotted from top to bottom are: the observed total flux spectrum in units of $10^{-17} \mathrm{erg} \mathrm{s}^{-1} \mathrm{~cm}^{-2} \AA^{-1}$ at two different scales, the first to show strong emission lines and the second to show the continuum. Shaded areas indicate regions of bright sky emission

to that of an unobscured quasar (Type $1 \mathrm{AGN}$ ) in both the slope of the continuum and the presence of broad emission lines (Vernet et al. 2001b). This is strong evidence for the presence of an obscured quasar in the nuclei of HzRGs. The spectral energy distribution of a typical quasar is bluer than most other components that contribute to the SED of HzRGs between the UV and near-IR. The dilution by the other components is smaller in the rest-frame UV. At $z>2$, this waveband is redshifted into the optical, where the most sensitive (spectro-)polarimeters exist on large telescopes, allowing detailed studies despite the cosmological brightness dimming.

The polarisation angle is in most cases closely perpendicular to the radio structure, indicating that the scattering occurs within the cones traced out by the radio jets (Vernet et al. 2001b). The spatially resolved narrow emission lines (see Sect. 3.2) are 
generally not polarised (e.g. Fig. 10), indicating that the scattering medium must be located between the broad and narrow line regions.

The wavelength dependence of the polarised emission is consistent with both grey dust or electron scattering. However, electron scattering can be excluded as it is much less efficient and would imply masses of the scattering medium which are close to the total galaxy mass (Manzini and di Serego Alighieri 1996). A clumpy scattering dust medium can also produce the observed continuum slope. Using spectropolarimetric observations near the rest frame $2200 \AA ̊$ dust feature, Solórzano-Iñarrea et al. (2004) argue that the composition of the dust is similar to that of Galactic dust.

Cimatti et al. (1993) presented a dust-scattering model that explains the structure, the polarisation properties and the spectral energy distribution of the ultraviolet aligned light with optically thin Mie scattering of quasar radiation emitted in a cone of $\sim 90^{\circ}$ opening angle. The required amount of spherically distributed dust is (1-3) $\times 10^{8} \mathrm{M}_{\odot}$, consistent with the estimate for the dust mass from the sub-millimetre data (Sect. 3.5.1). However, more recent predictions (Vernet et al. 2001b) indicate that the dust responsible for scattering the AGN emission should have a sub-millimetre emission that is an order of magnitude fainter than observed.

Not all HzRGs have significant polarisation. Two of eight objects studied by Vernet et al. (2001b) had fractional polarisation $f_{p}<2.4 \%$. There is no evidence that the nuclei of these HzRGs contain obscured quasars (Sect. 5.1). We discuss information derived from the UV polarisation observations about the obscured or dormant quasar in Sect. 5.1.

\section{STARS}

Captain Boyle: "I ofen looked up at the sky an' assed meself the question-what is the stars, what is the stars?"

Joxer Daly: "Ah, that's the question, that's the question-what is the stars?"

Act 1 "Juno and the Paycock", Seán O'Casey, Irish playright (1924)

During the last 15 years there has been mounting evidence that stars are a major constituent of HzRGs. Stellar signatures include (i) a plateau in the spectral energy distribution (SED) between 1 and $2 \mu \mathrm{m}$ characteristic of stars-the "1.6 $\mu \mathrm{m}$ bump" (Simpson and Eisenhardt 1999; Seymour et al. 2007) (Fig. 1), (ii) the detection of rest-frame UV stellar absorption lines in a few HzRGs (Dey et al. 1997) and (iii) measurement with the HST that the morphologies of several HzRGs follow de Vaucouleurs profiles. (e.g. van Breugel et al. 1998; Pentericci et al. 2001; Zirm et al. 2005) (Fig. 11).

In principle, spectral energy distributions (SEDs) are a powerful diagnostic of stellar populations and galaxy evolution. Young $(<0.5 \mathrm{Gyr})$ stars dominate the SED in the UV, whereas the SED longward of 4,000 A gives information about populations older than $\sim 1$ Gyr. However, disentangling the stellar contribution to the SEDs from the non-stellar components is complicated (see Fig. 1). At rest-frame UV wavelengths, contributions from scattered quasar light and nebular continuum must be taken into account, whereas re-radiation by dust contributes to the SED at far infrared and millimetre wavebands (e.g Fig. 1). 


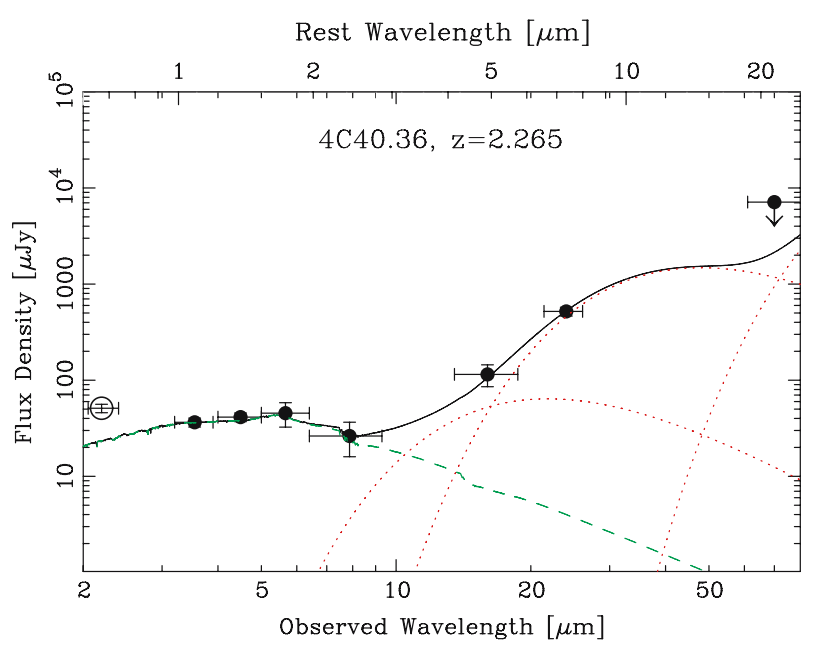

Fig. 11 The IR SED of 4C40.36 at $z=2.265$, with modelling, using data taken with IRAC and MIPS on the Spitzer Telescope [from Seymour et al. (2007)]. The solid dark line indicates the total best-fit SED. The stellar and dust components are indicated by dashed and dotted lines, respectively. Note the plateau, corresponding to the stellar " 1.6 $\mu \mathrm{m}$ bump" feature (Simpson and Eisenhardt 1999)

\subsection{Old stars: HzRGs as the most massive galaxies}

The SEDs of old (> 1 Gyr) stellar populations peak in the near-IR. The galaxies with the largest K-band luminosities in the early Universe are HzRGs. Because old stars are the best tracers of stellar mass, the brightness of the observed $K$-band emission has long been used to argue that distant radio galaxies are very massive, with masses of up to $10^{12} \mathrm{M}_{\odot}$ (Rocca-Volmerange et al. 2004). This places them on the upper end of the stellar mass function over the entire redshift range $0<z<4$ (e.g. Rocca-Volmerange et al. 2004).

A major tool in HzRG research is the Hubble $K-z$ diagram, introduced by Lilly and Longair (1984). Despite significant spectral correction effects (K-corrections), radio galaxies form a remarkably narrow sequence out to $z \sim 3$, especially when compared with near-IR selected field galaxies (e.g. De Breuck et al. 2002) (Fig. 12, 13). The small scatter in the $K-z$ relation for HzRGs was found to be weakly correlated with radio luminosity (e.g. Best et al. 1998). Such a correlation can be understood if the radio power is dominated by the Eddington limiting luminosity of the nuclear supermassive black hole (Rawlings and Saunders 1991) (Sect. 5.2) and thus a measure of the black hole mass. The well-established relation between black hole and bulge mass (e.g. Magorrian et al. 1998) then implies that radio power would be correlated with host galaxy mass, as appears to be the case for low-redshift less-luminous radio galaxies (Best et al. 2005).

Caution should be exercised in determining the K-band continuum magnitudes for HzRGs and in interpreting the details of the $K-z$ diagram in terms of galaxy masses. There are several reasons for this. First, it is often difficult to disentangle the stellar component from the warm dust component of the K-band flux. Second, depending on 


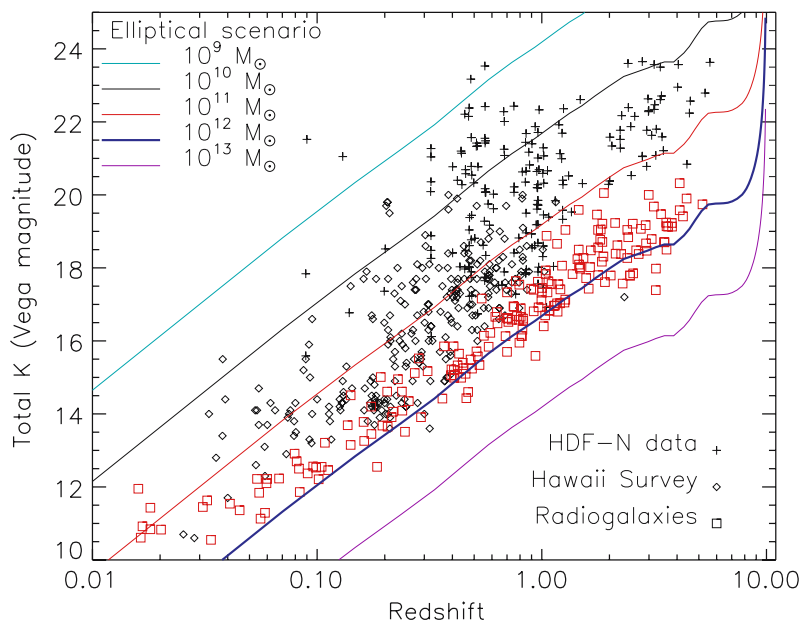

Fig. 12 Composite Hubble $K-z$ diagram of radio and optically selected galaxies, slightly modified from Rocca-Volmerange et al. (2004) and kindly provided to us by Brigitte Rocca. The radio galaxies are denoted by the red squares. The optically selected galaxies are plotted in black (HDFN $=$ crosses and Hawaii survey $=$ dots). The radio galaxies trace the upper envelope of the $K-z$ diagram, with HzRGs being amongst the brightest galaxies in the early Universe. Note that continuum fluxes derived from K-band or H-band magnitudes can be contaminated by bright emission lines in the band. Also plotted are K-magnitude tracks for elliptical galaxies formed from various initial reservoir gas masses (baryonic). These were calculated using the Pégase galaxy evolution models as described in Rocca-Volmerange et al. (2004)

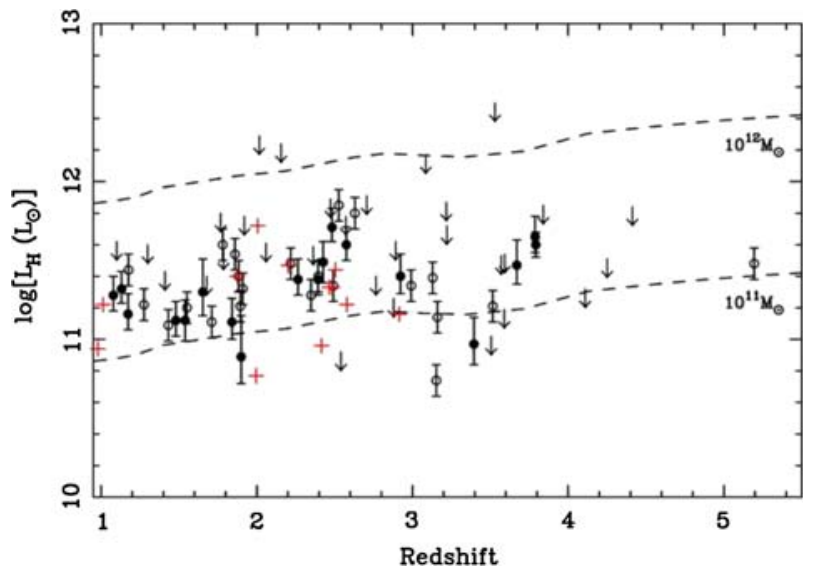

Fig. 13 Rest-frame H-band stellar luminosity vs. redshift for a sample of radio galaxies observed with the Spitzer Telescope. [From Seymour et al. (2007)]. The masses of stars are derived from the best-fit models to the multiband photometry, including Spitzer fluxes. Solid circles indicate HzRGs detected at 24-160 $\mathrm{m}$ with MIPS. Open circles are luminosities derived from HzRGs that were not detected by MIPS. Upper limits are indicated by arrows. Dashed lines correspond to elliptical galaxies formed at $z_{\text {form }}=10$. Crosses mark the stellar luminosity of sub millimetre galaxies

the redshift, there is also a contribution to the K-band emission from bright emission lines. Mass and age estimates derived from the uncorrected colours are therefore often overestimated (Eales and Rawlings 1996; Rocca-Volmerange et al. 2004). Third, the 
stellar population models on which the masses are based are subject to considerable uncertainty. For example, incorporation of recent data on thermally pulsating asymptotic giant branch stars reduce the derived masses by large factors (Bruzual 2007). In addition the Initial Mass Function and the gas to dust content at early epochs is highly uncertain.

Because of its ability to measure the rest-frame near-IR fluxes of HzRGs radio galaxies, the Spitzer Telescope, has facilitated considerable progress in this field during the last few years. Seymour et al. (2007) surveyed 69 radio galaxies having $1<z<5.2$ at 3.6, 4.5, 5.8, 8.0, 16 and $24 \mu \mathrm{m}$. They decomposed the rest-frame optical to infrared spectral energy distributions into stellar, AGN, and dust components and determined the contribution of host galaxy stellar emission at rest-frame H-band. By consistently deriving the stellar luminosity at the same rest-frame wavelength near the peak of the stellar emission at $\lambda_{\text {rest }} \sim 1.6 \mu \mathrm{m}$, effects of measuring the $K-z$ diagram with galaxies over a large range of redshift through fixed observing bands were eliminated and contamination of the fluxes by AGN and emission lines was minimised.

The Spitzer results confirm that radio galaxies have stellar masses mostly within the $10^{11}$ to $10^{12} \mathrm{M}_{\odot}$ range, with almost no dependence on redshift. The fraction of emitted light at rest-frame H-band from stars was found to be $>0.6$ for $\sim 75 \%$ of the HzRGs. The weak correlation of stellar mass with radio power determined using ground-based data is only marginally significant in the Spitzer results. Rest-frame near-IR studies of less luminous radio sources are needed to extend the range in radio luminosity and settle whether there is indeed a dependence of stellar mass on radio luminosity.

\subsection{Young stars-UV absorption lines}

HzRGs undergo vigorous star formation. The most direct evidence for this is the detection of rest-frame UV photospheric stellar absorption lines and P-Cygni features driven by stellar winds. Because observation of these lines requires several hour exposures on $10 \mathrm{~m}$-class telescopes, their detection is limited to the S V $\lambda 1502$ line in 4C 41.17 (Dey et al. 1997), tentative measurement of C III $\lambda 1428$ in TN J2007-1316 (De Breuck and Reuland 2005) and VLT spectroscopy of the Spiderweb Galaxy (Miley et al. 2006; Nesvadba et al. 2006; Hatch et al. 2007). The presence of absorption lines is direct evidence for prodigious star formation in 4C41.17 (up to $1,500 \mathrm{M}_{\odot} \mathrm{yr}^{-1}$, after correction for extinction) (Dey et al. 1997; Reuland et al. 2004). For radio galaxies whose rest-frame UV continuum is polarised, scattered AGN continuum emission (Vernet et al. 2001a), make it difficult to measure the star formation rates directly (Fig. 14).

Additional evidence that substantial star formation is occurring in HzRGs is provided by the sub-millimetre observations of dust, for whose heating star formation rates of thousands $M_{\odot}$ per year are needed (but see Sect. 3.5) and the systematically larger Ly $\alpha$ luminosities and $\mathrm{Ly} \alpha / \mathrm{HeII}$ ratios for HzRGs with $z>3$, than for those with $2<z<3$ (Villar-Martín et al. 2007a,b) (Sect. 3.2.2).

\subsection{The alignment effect-jet-induced star formation}

One of the most remarkable properties of $\mathrm{HzRG}$ is the approximate alignment between their radio and optical continuum emissions. This phenomenon was completely unex- 


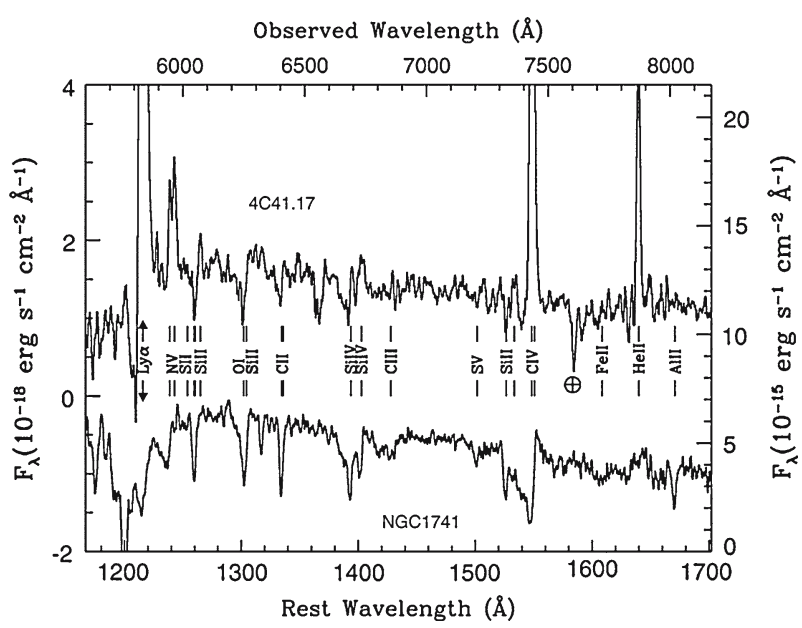

Fig. 14 Spectrum of the central $2 \times 1$ of the HzRG 4C 41.17 at $z=3.8$ [from Dey et al. (1997)]. This is compared with the UV spectrum of the B1 star-forming knot in the nearby Wolf-Rayet starburst galaxy NGC 1741 (Conti et al. 1996). The ordinate is labeled with the flux density scales for 4C 41.17 and NGC 1741B1 on the left and right axes respectively. The two spectra show many similarities in their absorption line properties

pected when it was discovered two decades ago (Chambers et al. 1987; McCarthy et al. 1987). The "alignment effect" sets in for redshifts $z \geq 0.7$. Several models have been proposed or considered to account for this alignments (e.g. McCarthy 1993). The two most promising models are (i) dust scattering of light from a hidden quasar at the nucleus of the HzRG (Tadhunter et al. 1998) and (ii) triggering of star formation by the synchrotron jet as it propagates outward from the nucleus (De Young 1989; Rees 1989; Begelman and Cioffi 1989; Bicknell et al. 2000). We have already seen that radio jets frequently interact vigorously with the warm gas in HzRGs.

Since the extensive review by McCarthy (1993), high resolution imaging with the HST has provided important new information on the morphologies of HzRGs and on the alignment effect (Pentericci et al. 1999; Bicknell et al. 2000; Pentericci et al. 2001). Images with the HST have revealed that HzRG hosts generally have clumpy optical morphologies (Pentericci et al. 1998, 1999).

Using HST observations of a sample of $20 \mathrm{HzRG}$ combined with similar resolution VLA radio maps, Pentericci et al. $(1999,2001)$ have for the first time examined the close relationship between the radio and rest-frame UV and optical morphologies. They find that the alignment effect extends into the rest-frame optical, with the $z>2.5$ radio galaxies displaying a much more clumpy structure than their lowredshift counterparts, which are well represented by de Vaucouleurs profiles. At a slightly lower resolution, near-IR imaging with the Keck Telescope (van Breugel et al. 1998) also suggest that the morphology changes from aligned clumpy structures at $z>3$ to relaxed de Vaucouleurs profiles at $z<3$. However, the redshift-dependance of luminosity and the change in the observing wavelength with redshift complicates the interpretation of these results. We note that the IR structures of B2/6C galaxies at 
$z \sim 1$ appear less aligned with their radio structures than are the brighter $3 \mathrm{C}$ galaxies at this redshift, implying that the strength of the alignment effect depends on radio luminosity (Eales et al. 1997).

It is now clear that no single mechanism can completely account for all the observed properties of the aligned emission. The occurrence of optical polarisation in HzRGs in some but not all HzRGs is evidence that dust scattering plays a role. However, scattering cannot explain the observed detailed optical morphologies and similar bending behaviour that has been observed between the optical and radio structures. Neither can dust scattering account for the alignment of the continuum emission redwards of the rest frame $4000 \mathrm{~A}$ break. The alignment effect is most likely due to a combination of star formation induced by the jet and scattering of hidden quasar light along the radio source.

The most comprehensive study of jet-induced star formation has been made for 4C41.17 at $z=3.8$ by Bicknell et al. (2000). The interaction of a high-powered $\left(\sim 10^{46} \mathrm{erg} \mathrm{s}^{-1}\right)$ jet with a dense cloud in the halo of $4 \mathrm{C} 41.17$ is shown to produce shock-excited emission lines and induce star formation. Such shock-initiated star formation could proceed on a timescale of a few $\times 10^{6} \mathrm{yr}$ ), i.e well within the estimated lifetime of the radio source (few $\times 10^{7} \mathrm{yr}$ ) (Fig. 15).

Studies of several low-redshift radio galaxies provide further evidence that jetinduced star formation occurs. These include NGC 541/Minkowski's Object (3C40) (van Breugel et al. 1985a; Croft et al. 2006), 3C 285 (van Breugel and Dey 1993) and the closest radio galaxy Centaurus A (Graham 1998). Such effects can be expected to be much more prevalent during the era of the Universe at $z>2$, when every galaxy may well have undergone nuclear activity.

If the red aligned emission is indeed produced by stars, their ages would be $>1 \mathrm{Gyr}$, implying that this mode of star formation has operated almost since the formation of the host galaxies. It would also require that the radio jet was active and aligned in approximately the same position angle for this length of time. van Breugel et al. (1998) suggested that the tendency of HzRGs to be clumpier at $z>3$ is due to the increased importance of jet-induced star formation at earlier epochs. We know little about how the first population of stars in galaxies were made. Although unconventional, it is tempting to speculate that jet-induced star formation played a significant role in producing stars during the first $3 \mathrm{Gyr}$ after the Big Bang. Gopal-Krishna et al. (2004) have suggested that a large fraction of all proto-galactic material within the cosmic web was impacted by the expanding lobes of radio galaxies during the quasar era, triggering star formation.

\section{Active galactic nucleus (AGN)}

"Power exercised in secret, especially under the cloak of national security, is doubly dangerous" US Senator, W. Proxmire

Because the luminous collimated radio emission must have been generated in their cores, the nuclei of all HzRGs must have been active at some time in their histories. 


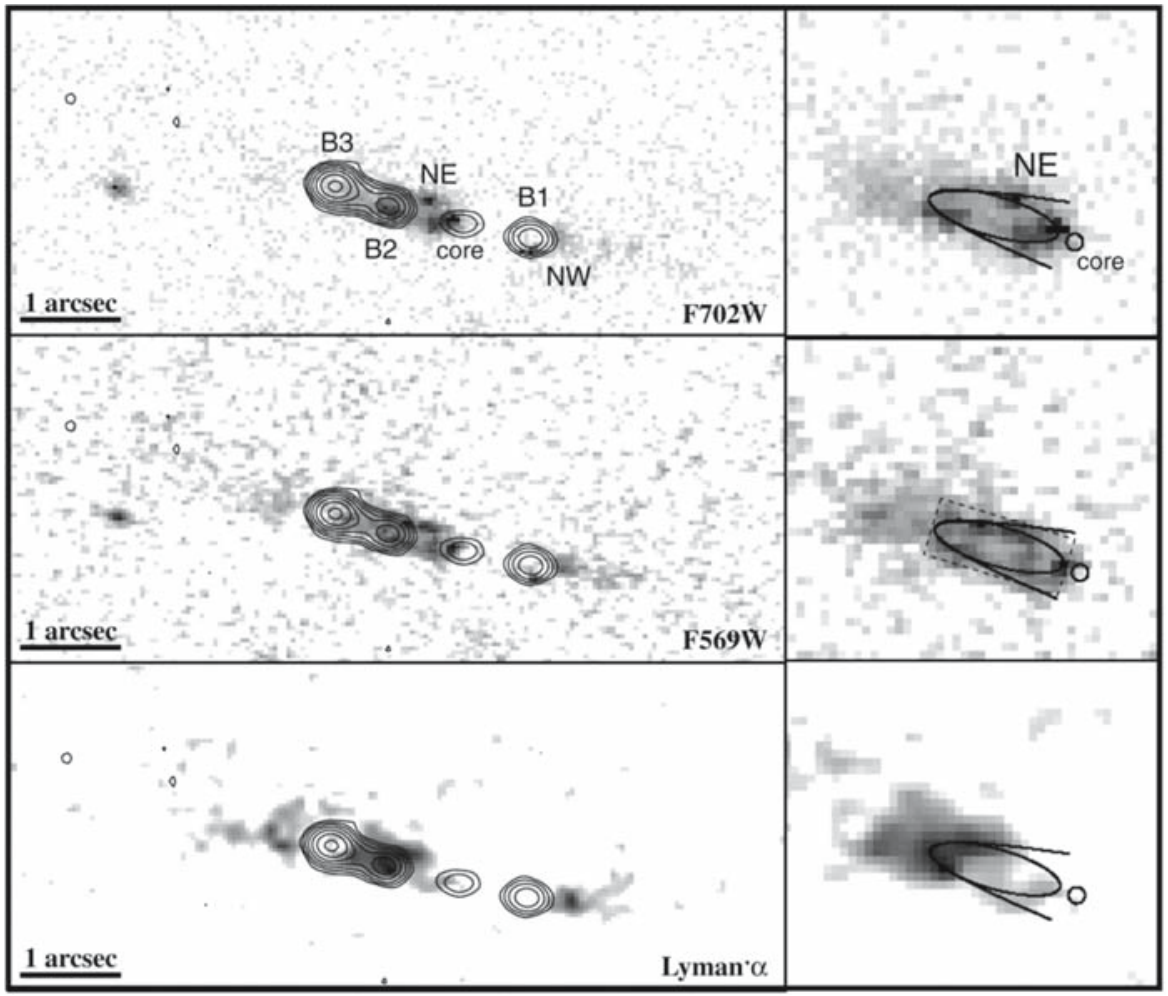

Fig. 15 Evidence for jet-induced star formation in 4C41.17 at $z=3.8$ [from (Bicknell et al. 2000)]. Montage of three HST images taken through the F702W, F569W (continuum), and Ly $\alpha$ filters. The $8 \mathrm{GHz}$ radio images of Carilli et al. (1994) are superimposed as contours. The absence of significant optical polarisation and the details of the optical-radio morphologies cannot be explained by the scattering models. The Ly $\alpha$ image shows a bright arc-shaped feature near B2 at the apex of the edge-brightened UV structure, suggestive of a strong bow shock at the location where the jet interacts with dense ambient gas (see also right-hand panels). Bicknell et al. (2000) suggest that this is manifest through the associated shock-excited line emission and star formation in the bifurcated structure

\subsection{Hidden or dormant quasar-The Unification Church}

The most extreme form of nuclear activity is seen in quasars, for which the nuclei are so bright that the galaxies are observed as "quasi-stellar objects". There is strong evidence that radio galaxies and quasars are manifestations of the same parent population. When we observe objects to be radio galaxies, the associated quasars are believed to be either (i) hidden due to our special viewing angle and/or (ii) highly variable and observed when they are in a dormant state.

The most widely accepted models for unifying quasars and radio galaxies postulate that their observed properties are dependent on the orientation of the radio source axes relative to us, the observers. For a review of unification via orientation, see Antonucci (1993); Urry and Padovani (1995) and Urry (2004). One of the key factors in establishing the validity of HzRG unification through orientation has been the signature of a 
hidden quasar detected in some radio galaxies in polarised light. Polarimetry with the Keck Telescope (Vernet et al. 2001b) (See Sect. 3.5.2) show that HzRGs are usually, but not always, highly polarised just redward of Ly $\alpha$ and that dust-reflected quasar light appears generally to dominate their rest-frame ultraviolet continua. Furthermore, broad emission lines, with equivalent widths similar to those seen in quasars, are detected in the two most polarised HzRGs in the Vernet et al. (2001b) sample of nine objects.

There is a convincing case that an obscured quasar exists in those HzRGs for which high fractional polarisations and broad polarised recombination lines have been measured. What about HzRGs with low polarisation? For these objects, it is possible that active quasars are present that are even more highly obscured than the polarised HzRGs. However an equally plausible explanation is that the quasars are dormant during the epoch of observation (van Groningen et al. 1980). We know that quasars are highly variable, varying by as much as 2 magnitudes during a day. Quasar variability has been studied on timescales of up to 50 years (de Vries et al. 2006). The variability appears to be intrinsic to the AGNs, and occurs in flares of varying timescales, possibly due to accretion-disk instabilities.

During the lifetime of an extended radio sources (at least a few $\times 10^{7}$ years), quasar activity can be expected to vary considerably. Knots in the radio morphologies can be interpreted as tracing bursts of quasar activity in the radio nuclei (Miley 1980). According to such a dormant quasar model, if the flare duration is substantially shorter than the quiescent periods the objects would be more likely to be observed as radio galaxies than radio-loud quasars. Also if the flares recurred on a timescale shorter than the recombination time $\left(\sim 10^{4}-10^{5}\right.$ year), the excitation of the gas would persist. A recurrently flaring quasar could also be a source of excitation for some of the nonradio Ly $\alpha$ halos (Sect. 3.2.5).

In summary, there is strong evidence that quasars exist in the nucleus of all HzRGs. Although orientation and varying intrinsic activity both probably contribute to the observed properties, their relative importance is still unclear.

\subsection{Supermassive black hole_-powerhouse of the AGN}

Extragalactic radio sources, and all active galactic nuclei, are believed to be powered by gravitational energy produced by rotating supermassive black holes (SMBHs) in their nuclei (e.g. Lynden-Bell 1969). Material in the galaxies is accreted onto the SMBHs, converted into kinetic energy and ejected as collimated relativistic jets along the rotation axes of the SMBHs to produce the radio sources. For a theoretical review of this field, see Blandford (2001). Although there is a wide agreement about the general idea, many of the details are not well understood.

Observational evidence for the widespread occurrence of SMBHs in galactic nuclei includes (i) spectroscopy of the gigamaser in the nucleus of NGC 4258 (Miyoshi et al. 1995) (ii) spectroscopy and photometry of galactic nuclei with the HST leading to the correlation between the masses of galaxy bulges and SMBHs in early type galaxies (e.g. Magorrian et al. 1998; Kormendy and Gebhardt 2001; Häring and Rix 2004; Novak et al. 2006), (iii) the small collimation scale of radio sources, measured with 
VLBI $\left(10^{17} \mathrm{~cm}\right.$; Junor et al. 1999) and (iv) the similarity of the large-scale and smallscale orientations of some giant radio sources, indicating that the "memory" of collimation axis can persist for as long as $\sim 10^{8}$ year (Fomalont and Miley 1975; Schilizzi et al. 1979; Miley 1980).

Because of the interaction of the radio jet with the gas, line widths in the central regions of HzRGs cannot be used to derive the masses of the presumed SMBHs in their nuclei, as for radio-quiet galaxies. The empirical correlation of SMBH with the bulges of early type galaxies (Häring and Rix 2004) would predict black hole masses of $\sim 10^{9} \mathrm{M}_{\odot}$ at the centre of a $10^{12} \mathrm{M}_{\odot}$ HzRG. McLure and Jarvis (2002) derived similar masses for SMBHs in radio-loud quasars from the width of their emission lines. However, such estimates should be treated with caution. There is a possibility that the widths of the emission lines are widened due to pressure from the radio jets. Also, little is known about how the SMBHs assemble during the formation and evolution of a massive galaxy, such as HzRG, so extrapolation of results from lower redshifts may not be valid. Silk and Rees (1998) postulated that supermassive black holes form within the first sub-galactic structures that virialise at high redshift, and are in place before most galactic stars have formed.

\subsection{Extinction of the "dinosaurs"}

Luminous quasars and radio galaxies have undergone strong evolution in their space densities since $z \sim 2$ (the peak of the "quasar" era") and both species are now virtually extinct (Sect. 1.4). The reason why such extreme manifestations of nuclear activity are no longer with us is not fully understood. One obvious explanation is that the supply of "food" for the SMBHs began to diminish as more and more of the the gas in massive galaxies was converted to stars (e.g. Menci 2004).

\section{Nature of HzRG hosts-massive forming galaxies}

Viktor Ambartsumian: "It is easy to show applying statistical considerations, that the galaxies NGC 5128 (Centaurus A) and Cygnus A cannot be the result of the collisions of two previously independent galaxies"

Rudolf Minkowski "Results of a detailed investigation of NGC 1275 (Perseus A), reported at this symposium, admit to no other interpretation than that this system consists of two colliding galaxies”, IAU Symposium No. 5, Dublin, 1955

Having considered the various building blocks of HzRGs in detail, we now address the nature of HzRGs and how these objects fit into general schemes of galaxy evolution.

There are several reasons for concluding that HzRGs are massive forming galaxies. First, we have seen that their large near-IR luminosities imply that HzRGs are amongst the most it massive galaxies in the early Universe (Sect. 4.1). Second, HzRG hosts have clumpy UV continuum morphologies (Sect. 4.3), as expected from galaxies forming through mergers and in accordance with hierarchical models of massive galaxy evolution (Larson 1992; Dubinski 1998; Gao et al. 2004; Springel et al. 2005b). Third, 
the rest-frame UV spectra and millimetre SEDs indicate that HzRGs are undergoing copious star formation (e.g. Dey et al. 1997) (Sect. 4.2).

Not only are HzRGs massive forming galaxies, but there is also a strong evidence that they are the progenitors of the most massive galaxies in the local Universe, i.e. the giant galaxies that dominate the central regions of rich local galaxy clusters. We have seen that HzRGs are generally embedded in giant (cD-sized) ionised gas halos (Sect. 3.2). In Sect. 7 we shall show that they are often surrounded by galaxy overdensities, whose structures have sizes of a few Mpc (Sect. 7). For all these reasons, HzRGs are the probable ancestors of brightest cluster galaxies (BCGs) and cD galaxies.

What can studying the development of HzRGs tell us about the evolution of massive galaxies in general? Recently, two processes have been invoked to explain discrepancies between the observed colour and luminosity statistics of faint galaxies and the model predictions. In contrast with what might be expected from simple hierarchical merging scenarios, the data imply that star formation occurs earlier in the history of the most massive galaxies than for the less massive ones. This process is called galaxy “downsizing” (Cowie et al. 1996; Heavens et al. 2004; Thomas et al. 2005; Panter et al. 2007). AGNs are preferentially found in the most massive galaxies. To quench star formation in these galaxies, it has been proposed that there is some sort of feedback mechanism, in which the AGN influences the star formation history of the galaxy. This AGN feedback (Di Matteo et al. 2005; Best et al. 2005; Springel et al. 2005a; Croton et al. 2006; Hopkins et al. 2006; Best 2007) provides modelers with additional parameters that, not surprisingly, gives a better fit to the data. The potential importance of AGN feedback for galaxy formation processes was only realised after it was shown that every galaxy may host a supermassive black hole (Sect. 5.2), establishing a general link between galaxies and AGN.

As the most massive known galaxies in the early Universe and the seat of powerful AGN, HzRGs are important laboratories for studying the processes responsible for downsizing and AGN feedback. In practice, feedback scenarios are complicated and involve several physical processes that occur simultaneously. We have seen in Sects. 2, 3,4 , and 5 that there is considerable evidence for physical interaction between the various building blocks of HzRGs - relativistic plasma, gas, dust, stars and the AGN. As they merge, the satellite galaxies will interchange gas with the ambient medium in the system. The gas will move inwards through cooling flows and accretion and provide fuel for the supermassive black hole. The SMBHs generate quasars and relativistic jets. Hidden and/or dormant quasars heat the dust. The jets together with superwinds from starbursts (Armus et al. 1990; Zirm et al. 2005) drive gas outwards (Nesvadba et al. 2006) and can trigger star formation. Shocks will be rampant in the chaotic environments in which these processes are competing with each other.

Two modes have been distinguished in theoretical models for coupling of the energy output of the AGN to the surrounding gas, with rather confusing names. "Quasarmode" feedback is defined as the situation when radiative coupling occurs that expels the gas and thereby quenches star formation in the forming galaxy (Hopkins et al. 2006). In "radio-mode" coupling (Best 2007), star formation is slowed down due to the mechanical energy of the synchrotron radio jets that inhibits cooling of the ambient gas. However, such rigid distinction between feedback processes are highly simplified (e.g. Fu and Stockton 2007). 


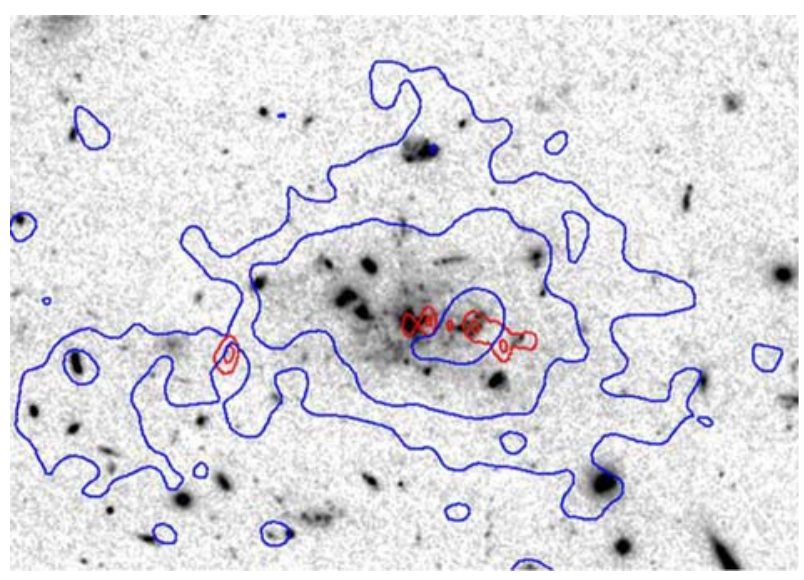

Fig. 16 The Spiderweb Galaxy. Deep Hubble image of the core of the MRC 1138-262 protocluster at $z=2.2$ obtained with the Advanced Camera for Surveys. [From Miley et al. (2006)]. Superimposed on the HST image are contours of Ly $\alpha$ (blue, resolution 1") obtained with ESO's very Large Telescope (VLT), delineating the gaseous nebula and radio $8 \mathrm{GHz}$ contours (red, resolution $0.3^{\prime \prime}$ ) obtained with NRAO's VLA, delineating the non-thermal radio emission. The gaseous nebula extends for $>200 \mathrm{kpc}$ and is comparable in size with the envelopes of $\mathrm{cD}$ galaxies in the local Universe

It is impossible to understand how these complicated AGN feedback processes influences the evolution of massive galaxies from a consideration of statistical data alone.

We shall now illustrate some of these processes and their relevance to galaxy formation by considering a specific example of a HzRG in more detail.

\subsection{The Spiderweb Galaxy—a case study}

The Spiderweb Galaxy (MRC 1138-262) at a redshift of $z=2.2$ is one of the most intensively studied HzRGs (Miley et al. 2006). This object provides a useful case study for illuminating several important physical processes that may occur generally in the evolution of the most massive galaxies. Because the Spiderweb Galaxy is (i) relatively close-by, (ii) one of the brightest known HzRGs and (iii) is the HzRG with the deepest HST optical image, it is an important laboratory for testing simulations of forming massive galaxies at the centers of galaxy clusters.

This large galaxy has several of the properties expected for the progenitor of a dominant cluster galaxy (Pentericci et al. 1997, 1998, 2000a, 2001). The host galaxy is surrounded by a giant Ly $\alpha$ halo (Pentericci et al. 2000a; Kurk et al. 2004b,a) and embedded in dense hot ionised gas with an ordered magnetic field (Carilli et al. 1998). The radio galaxy is associated with a $3 \mathrm{Mpc}$-sized structure of galaxies, of estimated mass $>2 \times 10^{14} \mathrm{M}_{\odot}$, the presumed antecedent of a local rich cluster (Sect. 7).

The beautiful ACS Hubble image of the Spiderweb Galaxy is shown in Fig. 16, with the radio source and Ly $\alpha$ halo superimposed. The figure illustrates the structures of the radio, warm gas and stellar components in a relatively nearby HzRG.

It also provides dramatic evidence that tens of satellite galaxies were merging into a massive galaxy, $\sim 10 \mathrm{Gyr}$ ago. The morphological complexity and clumpiness agrees 
qualitatively with predictions of hierarchical galaxy formation models (Larson 1992; Kauffmann et al. 1993; Baugh et al. 1998; Dubinski 1998; Gao et al. 2004; Springel et al. 2005b), and illustrates this process in unprecedented detail. Ly $\alpha$ spectroscopy shows relative velocities of several hundred $\mathrm{km} \mathrm{s}^{-1}$, implying that the satellite galaxies ("flies") will traverse the $100 \mathrm{kpc}$ extent of the Spiderweb many times in the interval between $z \sim 2.2$ and $z \sim 0$, consistent with the merger scenario.

An intriguing aspect of the Spiderweb Galaxy is the presence of faint diffuse emission between the satellite galaxies (Hatch et al. 2007). Approximately 50\% of the ultraviolet light from the Spiderweb Galaxy is in diffuse "intergalactic" light, extending over about $60 \mathrm{kpc}$ diameter halo. The luminosity in diffuse light implies that the emission is dominated young stars with a star formation rate of $>80 M_{\odot} \mathrm{yr}^{-1}$. Under reasonable assumptions, the diffuse emission seen in the Spiderweb Galaxy could evolve into the $\mathrm{CD}$ envelopes seen in many dominant cluster galaxies seen at low redshifts.

The total mass of all the flies in the Spiderweb, derived from their UV luminosities (assuming 1 Gyr starbursts), is less than a tenth of the mass of the whole galaxy obtained from its IR luminosity (Miley et al. 2006). Because the UV emission is produced by ongoing star formation and the IR emission by old stars, this implies that most of the galaxy mass may already have assembled by $z \sim 2.2$, consistent with downsizing scenarios.

Merging, downsizing and feedback are all likely to be occurring simultaneously in the Spiderweb Galaxy. Merging is a plausible fueling source for the nuclear supermassive black holes that produce the radio sources. Pressure from these radio sources is sufficient to expel a large fraction of gas from the galaxies (Nesvadba et al. 2006), thereby quenching star formation (Croton et al. 2006). Because radio lifetimes are relatively short (few $\times 10^{7}$ years), all massive ellipticals may have gone through a similar short but crucial radio-loud phase during their evolution.

An unexpected feature of the HST image is that there is a significant excess of faint satellite galaxies with linear structures (Miley et al. 2006). These galaxies (linear "flies") have similar morphologies (e.g. chains and tadpoles) to the linear galaxies that dominate resolved faint galaxies $\left(i_{775}>24\right)$ in the Hubble Ultra Deep Field (UDF) (Elmegreen et al. 2005; Straughn et al. 2006). Although linear galaxies must be an important constituent of the earliest galaxy population, their nature is poorly understood. Their presence in a merging system is relevant for theories of their formation. In the Spiderweb Galaxy the motions of the flies with velocities of several hundred $\mathrm{km} \mathrm{s}^{-1}$ through the dense gaseous halo, perturbed by superwinds from the nucleus (Armus et al. 1990; Zirm et al. 2005) and the radio jet, would result in shocks. The shocks would then lead to Jeans-unstable clouds, enhanced star formation along the direction of motion and to chain and tadpole morphologies (Taniguchi and Shioya 2001; Miley et al. 2006).

\section{Environment of HzRGS—protoclusters}

"We have traced the broad outline of the development in regions of exceptional fruitfulness of the first settled village communities into more populous towns and cities" 
"The city community arose around the altar of the seed-time blood sacrifice." The Outline of History, H. G. Wells, 1920

Within standard Cold Dark Matter (CDM) scenarios the first stars and stellar systems should form through gravitational infall of primordial gas in large CDM halos (e.g. White and Rees 1978). Simulations suggest that these halos merge and form web-like networks traced by young galaxies and re-ionised gas (e.g. Baugh et al. 1998; De Lucia and Blaizot 2007). The most massive galaxies, and the richest clusters emerge from regions with the largest overdensities.

\subsection{Finding protoclusters using HzRGs}

To investigate the emergence of large-scale structure in the Universe, it is important to find and study the most distant rich clusters of galaxies. Conventional methods for finding distant galaxy clusters usually rely on the detection of hot cluster gas using $\mathrm{X}$-ray techniques. These are limited by the sensitivity of X-ray telescopes to $\lesssim 1.3$, i.e. much smaller than the redshifts of HzRGs.

Since HzRGs are amongst the most massive galaxies in the early Universe, they are likely to inhabit regions that are conducive to the formation of rich galaxy clusters. During the mid-1990s observational evidence emerged that HzRGs are located in dense cluster-type environments. First, the measurement of large radio rotation measures (RM) around some HzRGs, indicated that the host galaxies are surrounded by a hot magnetised cluster gas (Carilli et al. 1997; Athreya et al. 1998b) (Sect. 3.1). Secondly, an excess of "companion galaxies" was found near HzRGs (Röttgering et al. 1996; Pascarelle et al. 1996; Le Fevre et al. 1996; Knopp and Chambers 1997). These results together with the indications that HzRGs hosts are forming dominant cluster galaxies (Sect. 6) prompted the initiation of direct searches for galaxy clusters in the vicinity of HzRGs.

Conventionally the term "galaxy cluster" refers to a bound structure of several hundred galaxies. At $z \sim 3$, the Universe is only about 2 Gyr old, i.e. insufficient time for a galaxy with a velocity of a few hundred $\mathrm{km} \mathrm{s}^{-1}$ to have crossed cluster-scale structures. Hence any overdense structure of galaxies observed at high redshifts must still be forming and cannot be virialised and bound. Following Overzier (2006), we shall use the term "protocluster" for an overdense structure in the early Universe $(z>2)$, whose properties are consistent with it being the ancestor of a local bound galaxy cluster.

During the last few years there have been several successful direct searches for protoclusters around HzRGs, with 8-10 m-class optical/infrared telescopes. One search technique is to use narrow-band imaging to detect emission line objects at redshifted wavelengths corresponding to those of the target HzRGs. A second technique is to carry out broad-band imaging with colours chosen to detect "dropout objects" due to redshifted features in the continuum spectral energy distributions. The protocluster candidate galaxies detected in these imaging experiments were generally followed up by multi-object spectroscopy to confirm their redshifts and their membership of the protoclusters. The most important emission line for establishing the redshifts of the protocluster galaxies is Ly $\alpha$ (Pentericci et al. 1997; Kurk et al. 2000; Venemans et al. 2002, 2004; Croft et al. 2005; Venemans et al. 2005, 2007). Other relevant emission 
lines for such searches for $z>3$ are $\mathrm{H} \alpha$ (Kurk et al. 2004a,b) or [OIII] $\lambda 5007$. There are two relevant features in the continuum spectra of galaxies that are exploited for such protocluster searches-the Lyman break around the $912 \AA$ Lyman continuum discontinuity (Miley et al. 2004; Overzier et al. 2006b,a; Intema et al. 2006) and the 4,000 ̊ break or Balmer break close to 3,648 ^ (Kurk et al. 2004b; Kajisawa et al. 2006; Kodama et al. 2007). The various galaxy detection techniques are complementary, because they tend to select stellar populations with different ages. For example, Ly $\alpha$ excess galaxies and Lyman break galaxies have young on-going star-forming populations, while the Balmer technique is sensitive to stellar populations that are older than a few $\times 10^{8}$ years.

The measured overdensities of Ly $\alpha$ emitters in the radio-selected protoclusters are factors of 3-5 larger than the field density of Ly $\alpha$ emitters at similar redshifts (Venemans et al. 2005, 2007). A recent study with the HST of 4,000 $\mathrm{A}$ break objects in MRC1138-262 at $z=2.2$, the protocluster that contains the Spiderweb Galaxy (Zirm et al. 2007, submitted to Ap.J) shows that the overdensity of red galaxies is 6.2, compared with non-protocluster fields. The photometric redshifts of galaxies in this field shows a significant "redshift spike" for $2<z<2.5$. Although such observations at $z>5$ are sensitivity limited, a significant overdensity of both Ly $\alpha$ emitters (Venemans et al. 2004) and Lyman break galaxies (Overzier et al. 2006b) has been established around TN J0924-2201 at $z=5.2$, the HzRG with the highest redshift known to date.

Possible protoclusters have also been detected in non-targeted optical surveys, with several overdense regions found at large redshifts in the form of "redshift spikes" or "filaments" (e.g. Steidel et al. 1998; Keel et al. 1999; Steidel et al. 2000; Möller and Fynbo 2001; Shimasaku et al. 2003; Hayashino et al. 2004; Matsuda et al. 2005 ; Steidel et al. 2005; Ouchi et al. 2005). Although caution must be exercised in deriving overdensities from the redshift distributions alone, because the effects of peculiar galaxy velocities can influence the apparent clumpiness (Monaco et al. 2005). However, the observed structures around the HzRGs have two additional ingredient expected of ancestors of rich clusters, namely (i) the presence of the HzRG hosts with properties expected of progenitors of dominant cluster galaxies and (ii) sharp peaks in the redshift distributions of Ly $\alpha$ emitters that lie close to the predetermined redshift of the radio galaxy. Taken together, the measured galaxy overdensities combined with the presence of HzRGs is strong evidence that the galaxy structures around HzRGs are indeed the ancestors of rich local clusters.

For some of the HzRG-selected protoclusters, there is additional evidence for a dense environment from excess counts at millimetre (Ivison et al. 2000; Smail et al. 2003; Stevens et al. 2003; De Breuck et al. 2004a; Greve et al. 2007) and X-ray (Pentericci et al. 2002; Overzier et al. 2005) wavelengths. Also, the X-ray counts indicate an enhanced AGN fraction in protoclusters compared to the field (Figs. 17, 18, 19).

\subsection{Properties of radio-selected protoclusters}

The largest and most comprehensive HzRG protocluster search project was based on a study of eight radio galaxies, with redshifts ranging from 2.2 to 5.2, using the narrow- 


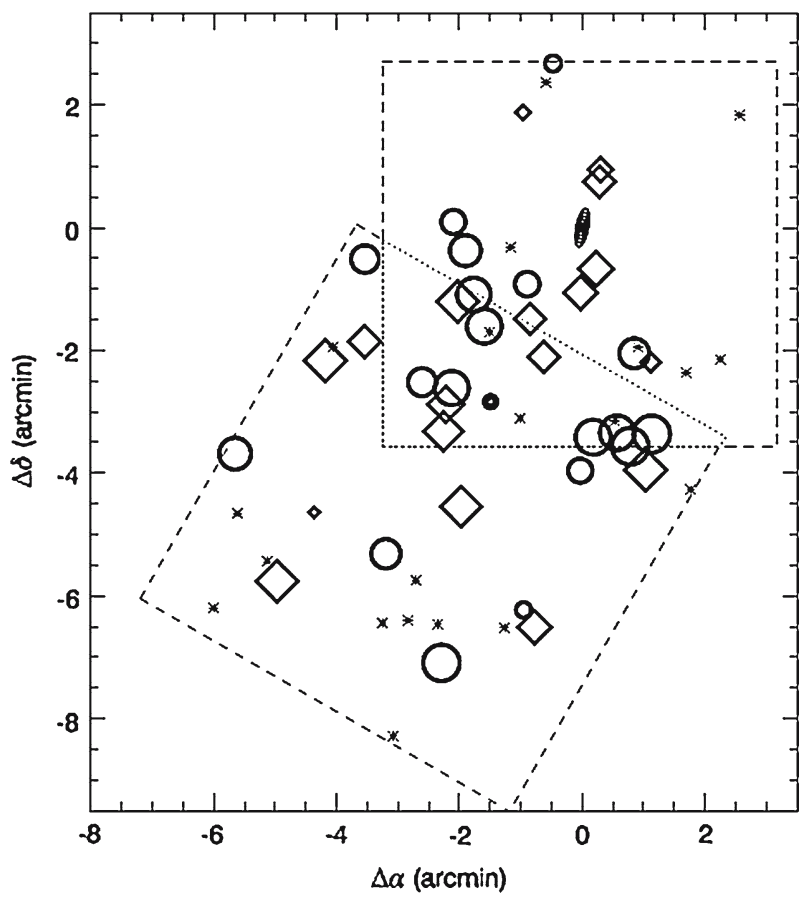

Fig. 17 HzRG-selected protocluster around TN J1338-1942 at z = 4.1. [From Venemans et al. (2002, 2007)]. Shown are the spatial distribution of the spectroscopically confirmed $z=4.1$ Ly $\alpha$ emitters (circles and diamonds), additional Ly $\alpha$ excess candidates from narrow-band imaging (stars) and the radio galaxy (square). The circles represent emitters with redshift smaller than the median redshift and the diamonds with those larger than the median. The size of the circles is scaled according to the relative velocity of the object. Larger circles and diamonds represent a larger relative velocity compared with the median velocity. The structure appears to be bound in the northwest of the image and unbound in the south

band Ly $\alpha$ search technique with the VLT (Pentericci et al. 2000a; Kurk et al. 2000; Venemans et al. 2002, 2004; Croft et al. 2005; Venemans et al. 2005, 2007). Followup observations have been made of candidates in several of the targets using a variety of ground-based telescopes as well as the Hubble and Spitzer Telescope (Kurk et al. 2004b,a; Miley et al. 2004; Overzier et al. 2005, 2006b; Intema et al. 2006; Kodama et al. 2007). Typically, 3 Mpc-scale regions around the HzRGs were covered in the Ly $\alpha$ searches. Ly $\alpha$ redshifts were determined for 168 objects. Almost all these objects are star-forming galaxies at similar redshift to the HzRG, with typical star formation rates of a few $\mathrm{M}_{\odot}$ per year, derived from the UV continuum and the Ly $\alpha$ luminosities.

Six of the 8 fields were found to be overdense in Lya emitters by a factor of 3-5 as compared to the field density of Ly $\alpha$ emitters at similar redshifts. The 6 included all of the most radio-luminous objects (i.e. all $\mathrm{HRzG}$ s with radio luminosities $L_{2.7 \mathrm{GHz}}>$ $6 \times 10^{33} \mathrm{erg} \mathrm{s}^{-1} \mathrm{~Hz}^{-1} \mathrm{sr}^{-1}$ ) (Venemans et al. 2007). Because these targets were chosen arbitrarily, (redshifts that place Ly $\alpha$ in an available narrow-band filter), it is possible that all HzRGs with such large radio luminosities are embedded in such overdensities.

Measured velocity dispersions are in the range $\sim 300-1,000 \mathrm{~km} \mathrm{~s}^{-1}$, centered within a few hundred $\mathrm{km} \mathrm{s}^{-1}$ of the mean velocity of the radio galaxies. Taking account of the 


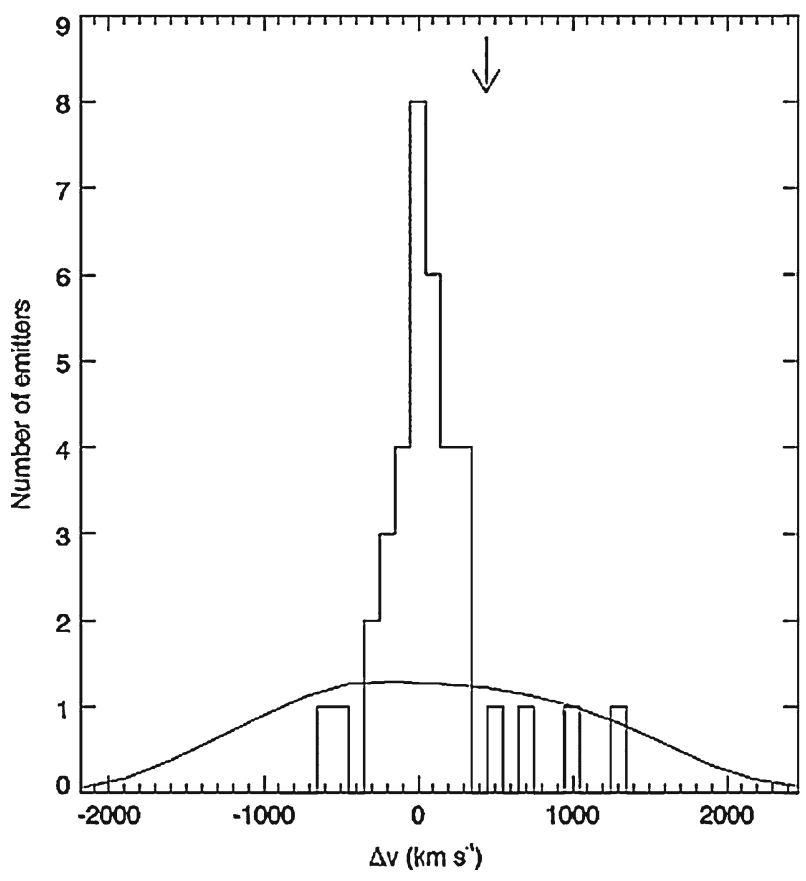

Fig. 18 HzRG-selected protocluster around TN J1338-1942 at $z=4.1$ as in Fig. 17. [From Venemans et al. (2002, 2007)]. Velocity distribution of the confirmed Ly $\alpha$ emitters. The peak velocity of the radio galaxy is indicated by an arrow. The solid line represents the selection function of the narrow-band filter normalised to the total number of confirmed emitters. Note that the velocity distribution of the detected emitters is substantially narrower than the filter width and centered within $200 \mathrm{~km} \mathrm{~s}^{-1}$ of the redshift of the radio galaxy. Similar data are available for six protoclusters
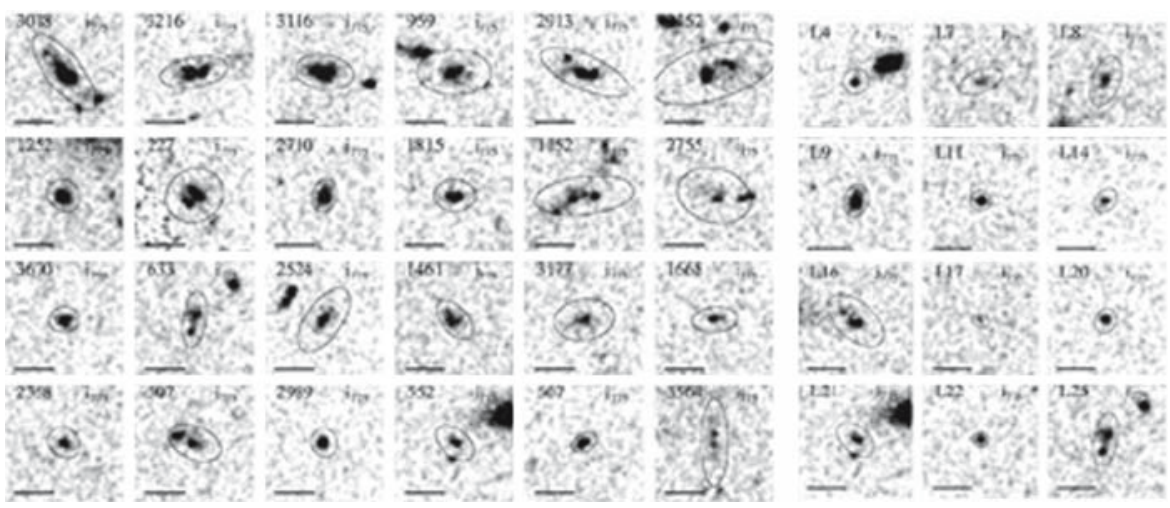

Fig. 19 Morphologies of galaxies in the protocluster TN J1338-1942 at $z=4.1$ with the ACS on the Hubble Space Telescope [From Overzier et al. (2006a)]. The filter is $i_{775}$ and each image measures $3^{\prime \prime} \times 3^{\prime \prime}$, corresponding to $\sim 20 \mathrm{kpc} \times 20 \mathrm{kpc}$ at $z \sim 4$. Left are images for a selection of the overdense $g_{475}$-dropout objects that are candidate Lyman break galaxies (LBGs) in the protocluster. Right are images of 12 spectroscopically confirmed Ly $\alpha$ excesses (LAE) protocluster members. There are a wide range of morphologies present, with some galaxies showing clear evidence for small-scale interactions 
relatively narrow peaks in the velocity distributions of the Ly $\alpha$ emitters compared with the widths of the imaging filters, the measured galaxy overdensities range from $\sim 5$ to 15 . Estimates for the sizes of the protocluster structures are limited by the typical size of the imaging fields used in the searches to date $\left(\sim 8^{\prime}\right.$ equivalent to $\left.\sim 3 \mathrm{Mpc}\right)$. Nevertheless, the protocluster sizes are estimated to be in the range $\sim 2-5 \mathrm{Mpc}$ (Intema et al. 2006; Venemans et al. 2007).

Because cluster-size overdense structures at high redshifts cannot be old enough to have become bound, the usual method of calculating cluster masses using the virial theorem cannot be applied. However, estimates for the masses of the protoclusters can be obtained from the volume occupied by the overdensity, the mean density of the Universe at the redshift of the protocluster, the measured galaxy overdensity, and the bias parameter (e.g. Steidel et al. 1998; Venemans et al. 2007). The masses obtained (a few times $10^{14}-10^{15} \mathrm{M}_{\odot}$ (Kurk et al. 2004a; Venemans et al. 2005)) are comparable to the masses of local rich clusters.

It is interesting to inquire what is the relation of the protocluster structures to the general large-scale structure of the Universe. Is the topology of the protocluster filamentary, or do HzRG-selected protoclusters illuminate the densest most tangled regions of the cosmic web. For TN J1338-1942 at redshift $z=4$.1, there are indications that the Mpc-sized protocluster of Ly $\alpha$ excess galaxies may be part of a larger-scale structure. A $25^{\prime} \times 25^{\prime}$ survey for Lyman break galaxy candidates (B-band dropouts) showed several significant density enhancements amidst large voids (Intema et al. 2006) and Overzier et al. (2006a)showed that this large scale structure ties in closely with significant sub-clustering across the $3^{\prime} \times 3^{\prime}$ ACS field near the radio galaxy.

Future wide-field narrow-band imaging and spectroscopy around HzRG-selected clusters should allow the topology of the Universe in the region of protoclusters to be mapped in detail, providing a glimpse of large-scale structure emerging in the early Universe for comparison with computer simulations.

\subsection{Are radio-selected protoclusters typical?}

We have seen that protocluster-like structures have been found around almost all of the most luminous radio galaxies with $z>2$ that have been targeted. However only $\sim 50 \%$ of powerful radio sources at $\mathrm{z} \sim 0.5$ are located in rich clusters and radio sources appear to avoid clusters at low redshift (Hill and Lilly 1991). What is known about the environment of radio galaxies having redshifts $1<z<2$ ? This range is difficult to study spectroscopically because of the redshift desert (Sect. 1.3). However, there have been several reports of detections of clusters and/or excesses of red galaxies around radio AGN in this redshift range (Hall et al. 1998; Kodama and Bower 2003; Nakata et al. 2002; Best et al. 2003a). We note that there is a strong correlation between the radio luminosities and redshift of objects in such studies due to Malmquist bias. Taking all the data together, there appears to be a substantial increase in the density of the environment around radio galaxies as a function of redshift and/or luminosity.

The statistics of the luminosity function of radio galaxies are consistent with every brightest cluster galaxy (BCG) having gone through a luminous radio phase during its evolution (Venemans et al. 2002, 2007). This statement is based on the facts (i) that the space density of luminous steep-spectrum radio sources decreases by $\sim 100$ 
between $2.5 \geq z>0$ (Sect. 1.4) and (ii) that the radio synchrotron lifetimes (few $\times 10^{7}$ year) are $\sim 100$ smaller than the cosmological time interval corresponding to the observed redshift range (few $\times 10^{9}$ year). Distant radio galaxies may therefore be typical progenitors of galaxies that dominate the cores of local clusters. Likewise, the ancestor of every rich cluster in the local Universe may have gone through a phase in which it hosted a HzRG. This would imply that HzRG-selected protoclusters are typical ancestors of local galaxy clusters.

\subsection{Protocluster evolution}

Radio-selected protoclusters are powerful laboratories for tracing the emergence of large scale structure and for studying the evolution of galaxies in dense cluster environments. An interesting parameter that can be measured for line-emitting galaxies is the velocity dispersion of the protoclusters. Although more statistics are needed, the velocity dispersion appears to decrease with increasing redshift (Venemans et al. 2007), consistent with the predictions from simulations of forming massive clusters (e.g. Eke et al., 1996).

A population study of the protocluster around the Spiderweb Galaxy PKS 1138-262 at $z=2.2$ was carried out by Kurk et al. (2004a,b), using deep optical and infrared observations with the VLT. Besides Ly $\alpha$ emitters, the study included objects having apparent $\mathrm{H} \alpha$ excesses and extremely red objects, with colours characteristic of old galaxies with 4,000 $\AA$ breaks at the redshift of the protocluster. An intriguing result of this study is that candidate $\mathrm{H} \alpha$ emitters and 4,000 $\AA$ break objects appear more concentrated towards the centre of the protocluster than the Ly $\alpha$ emitters. This indicates that the galaxies that are dominated by old stellar populations are more settled into the gravitational potential well of the protocluster, consistent with them being older.

An important diagnostic of galaxy and cluster evolution is the cluster colormagnitude diagram (CMD). The presence of a narrow "red sequence" in the cluster CMD is well established out to a redshift of 1.4 (Stanford et al. 2005). The red colors imply that the galaxies are dominated by older stars, whose SED peaks redwards of the $4,000 \AA$ break. The presence of this red sequence can be used to set a lower limit to the redshift at which the stellar populations formed. It is extremely difficult to measure the CMD at $z>1.4$, even with the largest ground-based telescopes.

However, indications of an emergent red sequence has been found in the CMD of the protocluster MRC1138-262 at $z=2.2$, both with a wide-field near-IR imager on the Subaru Telescope (Kodama et al. (2007); Fig. 20) and with the much narrow field but more sensitive NICMOS on the HST (Zirm et al. 2007). The galaxy colors indicate that while some relatively quiescent galaxies exist in the protocluster, most of the galaxies are still undergoing star formation Furthermore, Kodama et al. (2007) studied three other radio-selected protoclusters and found that the fraction of red galaxies in the four protoclusters increases between $z \sim 3$ and $z \sim 2$. To summarize, there is strong evidence that most protocluster galaxies undergo substantial star formation between $z \sim 3$ and $z \sim 2$ and that the bright end of the red sequence is still being formed during this epoch.

Information about the early evolution of stellar populations in clusters has been obtained at even higher redshifts. In the protocluster surrounding the HzRG TN J1338- 


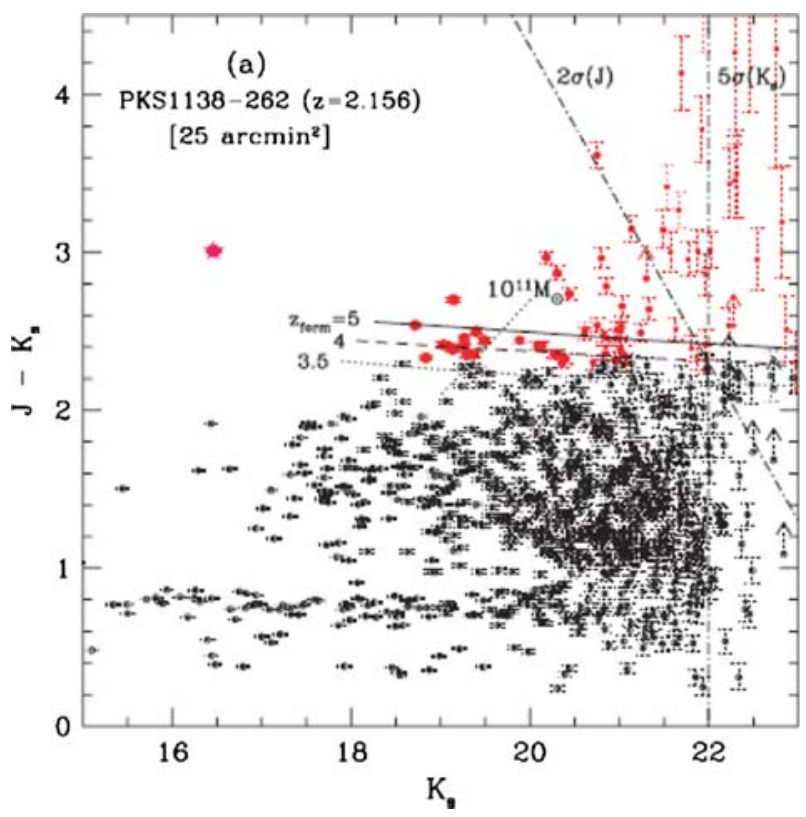

Fig. 20 Colour-magnitude diagram of $J-K_{S}$ plotted against $K_{S}$ for the MRC 1138-262 protocluster at $z=2.2$ [From Kodama et al. (2007)]. Filled circles indicate protocluster member candidates selected using color selection criteria. Large stars mark the targeted radio galaxies. Dotted error bars show 1s photometric errors. Solid, dashed and dotted lines show the expected location of the CMD at the relevant redshift for passive evolution. The iso-stellar mass lines of $10^{11}$ and $10^{10} \mathrm{M}_{\odot}$ are shown by thick dotted lines. The dot-dashed lines indicate $5 \sigma\left(\mathrm{K}_{S}\right)$ and $2 \sigma(J)$ detection limits

1942 at $z=4.1$, the distribution of Ly $\alpha$ emitters (LAEs) is highly filamentary and appears to avoid the locations of Lyman break galaxy candidates (Overzier et al. 2006a). A similar spatial segregation between LAEs and LBGs was observed in a structure around QSO SDSS J0211-0009 at $z=4.87$ (Kashikawa et al. 2007). This indicates that an age- or mass-density relation was emerging little more than $1 \mathrm{Gyr}$ after the Big Bang, when the Universe was only $10 \%$ of its present age.

We note that derived masses of $z>2$ LBGs and LAEs are $\gtrsim 10$ smaller than the masses of early-type galaxies in local clusters, indicating that a large fraction of the stellar mass still has to accumulate through merging (Overzier et al. 2006a). Detailed observations of protocluster regions on much larger scales ( $\sim 50$ co-moving $\mathrm{Mpc})$ are needed to test if the number density of LBGs is indeed consistent with forming the cluster red sequence population through merging. Simulations show that clusters with masses of $>10^{14} \mathrm{M}_{\odot}$ can be traced back to regions at $z=4-5$ of $20-40 \mathrm{Mpc}$ in size, and that these regions are associated with overdensities of typical dark matter halos hosting LAEs and LBGs of $\delta_{g} \sim 3$ and mass overdensities $\delta_{m}$ in the range 0.2-0.6 (Suwa et al. 2006). Also, recent numerical simulations of CDM growth predict that quasars at $z \sim 6$ may lie in the center of very massive dark matter halos of $\sim 4 \times 10^{12} \mathrm{M}_{\odot}$ (Springel et al. 2005a; Li et al. 2007). They are surrounded by many fainter galaxies, that will evolve into massive clusters of $\sim 4 \times 10^{15} \mathrm{M}_{\odot}$ at $z=0$. 
The addition of the new IR-oprical camera WFC3 to the HST and the advent of several new wide-field imagers and multi-object spectrographs on ground-based telescopes will allow the detailed evolution of protoclusters to be studied in great detail during the next few years.

\section{Conclusions and future}

"Curioser and curiouser!" cried Alice (she was so much surprised that for the moment she quite forgot how to speak good English). "Now I'm opening out like the largest telescope that ever was!” Alice's Adventures in Wonderland, Lewis Caroll, 1865

In this review we have shown that HzRGs are fascinating objects in their own right and that they provide important diagnostics for studying the early Universe. Knowledge about HzRGs and their evolution is fundamental to understanding the formation and evolution of galaxies and the large scale structure of the Universe.

There are still many aspects of HzRGs that are not understood. Here are some open questions.

- What is the particle acceleration mechanism in the relativistic plasma and why do HzRGs have much steeper radio spectra than nearby radio sources (Sect. 2.4).

- Why caused the most luminous radio galaxies and quasars to become virtually extinct between $\mathrm{z} \sim 2$ and the present (Sects. 1.4 and 5.3)?

- What is the nature, extent and kinematics of the hot gas, one of the most massive and least studied constituents of HzRGs (Sect. 3.1)?

- What is the origin of the Ly $\alpha$ halo that appears to be falling into the HzRG and how is this warm gas (filling factor $\sim 10^{-5}$ ) distributed with respect to the hotter and colder gas (filling factors $\sim 1$ ) (Sects. 3.2.4 and 2.4)?

- What are the detailed processes by which the radio jets interact with the gas and trigger starbursts and how important is jet-induced star formation for producing stars in the early Universe (Sects. 3.2.3 and 4.3)?

- What are the temperature and densities and composition of the molecular gas and dust? how is this distributed spatially and what are the implications for the star formation histories(Sects. 3.4 and 3.5)?

- What is the relative importance of AGN and starburst heating in the mid-IR emission of HzRG?

- What effect does galaxy merging have on star formation and what physical effects are responsible for downsizing (Sect. 6)?

- What effect does feedback between the AGN and the galaxy have on the evolution of HzRGs and the general evolution of massive galaxies (Sect. 6)?

- What is the detailed mechanism by which the SMBHs produce quasars and luminous collimated jets?

- How do the AGN/quasars vary on long timescales and what are the causes of the variability (Sects. 5.1 and 5.2)?

- How is the SMBH built up and what role does merging play in this evolution (Sect. 5.2)? 
- What is the size distribution of radio-selected protoclusters and what is is the topology of the cosmic web in the neighbourhood of the HzRGS (Sect. 7)?

- How do the various populations and constituents of distant protoclusters evolve and eventually become virialised clusters (Sect. 7.4)?

- What is the radio luminosity function of galaxies in radio-selected protoclusters? Is the radio emission from the protocluster galaxies an important contributor to forming the radio halos, that are seen at the centres of many nearby rich clusters? What is the strength and configuration of the protocluster magnetic fields (Sects. 7 and 2)?

There are good prospects for making progress during the next decade. Since its inception, the study of radio galaxies, has been observationally driven. Several forefront astronomical facilities now being constructed or planned that will give new insights into the nature of HzRGs and their environments.

First, with a combination of sensitivity and spatial resolution, the new low-frequency radio arrays, LOFAR (Röttgering et al. 2006) and the LWA (Kassim et al. 2006), will open up the frequency window below 50 MHz for HzRG studies. LOFAR will survey the sky to unprecedented depth at low-frequencies and will therefore be sensitive to the relatively rare radio sources that have extremely steep spectra. Because of the $\alpha$ vs $z$ relation (Sect. 2.4), LOFAR is likely to detect HzRGs at $z \sim 8$, if they exist. Studies of detailed low-frequency spectra and their spatial variations will provide new information about the mechanism responsible for the $\alpha$ vs $z$ relation. Presently combination of the new radio surveys with planned new deep optical and infrared wide-field surveys, such as PAN-STARRS (Hodapp et al. 2004) and those with the VST and VISTA (Arnaboldi et al. 2007) will be used to identify HzRGs and provide photometric data.

Another task for sensitive radio arrays, such as LOFAR, the EVLA (Napier 2006) and eventually the Square Kilometre Array (SKA), will be to survey the radio emission of galaxies in protoclusters. The new arrays will study radio emission produced by relativistic jets and be able to detect and investigate radio emission from the brightest star forming protocluster galaxies.

Second, ALMA (Brown et al. 2004) and the EVLA, with their unprecedented sensitivities and resolutions at millimetre and sub-millimetre wavelengths, will revolutionise the study of molecular gas and dust. Several different CO transitions can be observed, allowing entire "CO ladders" to be constructed and the density and temperature structure of the molecular gas to be unraveled. Fainter molecular lines can be used to trace even denser gas than that studied until now. Important information about the dust composition and the gas to dust ratios is likely to be obtained.

ALMA's sensitivity at millimetre wavelengths should also facilitate observations of the atomic CI Carbon lines in HzRGs. This would provide an important constraint on the global metalicity of the gas. The fine-structure line of $\mathrm{C}^{+}$at $\lambda_{\text {rest }}=157.74 \mu \mathrm{m}$ line is one of the main cooling lines in nearby galaxies, and has now also been detected in several of the most distant quasars known.

Third, we can expect considerable progress in disentangling the detailed evolutionary history both of HzRGS and of radio-selected protoclusters. This evolutionary detective work will be pursued by combining spectroscopic data from the next gen- 
eration of spectrographs on 8-m-class telescopes with imaging results from the new camera, WFC3 (Leckrone et al. 1998), on the Hubble Space Telescope. For example, the detection of supernovae in $z \sim 2$ protoclusters will become possible. On a longer timescale, tracing the detailed history of the formation and evolution of HzRGS and the surrounding protoclusters will be helped enormously with the advent of 30-m-class ground-based telescopes in the optical and near-infrared and the James Webb Space Telescope (JWST) (Gardner et al. 2006) in the near and mid-infrared.

Fourth, the next generation X-ray telescope, such as XEUS (Bavdaz et al. 2006) or Constellation-X (Tananbaum 2006), will make observations of X-rays from HzRGs an important tool for studying galaxy formation. It will have sufficient sensitivity to perform spectroscopic studies of hot gas in HzRGs. The $0.3-10 \mathrm{keV}$ X-ray band contains the inner (K-shell) lines for all of the abundant metals from carbon to zinc as well as many L-shell lines. These atomic transitions provide important new plasma diagnostics of the HzRG hot gas.

Fifth, and perhaps most exciting, the potential discovery of HzRGS with $\mathrm{z}>6$ could open up a unique new window for studying the very early Universe during the "Epoch of Reionisation". Recent observational constraints on suggest that cosmic reionisation may have taken place between $\mathrm{z} \sim 11$ and $\mathrm{z} \sim 6$ (Fan et al. 2006). The existence of HzRGs within the near edge of cosmic reionisation could be used as sensitive probes of intermediate- to small-scale structures in the neutral IGM, through redshifted HI absorption observations (Sect. 3.3.1) (Carilli et al. 2002b; Furlanetto and Loeb 2002), complementary to the very large scale that can be studied in HI emission.

Finally, we point out that in trying to understand HzRGs or in using them to probe the properties of the early Universe, we should exercise humility, remembering that $96 \%$ of the energy density in the universe is in a form of dark matter and dark energy, that are not directly observable.

HzRGS pinpoint the progenitors of cosmic megacities in the local Universe. Studying them is akin to investigating how human civilisations were urbanised. During the last few decades we have obtained some glimmerings of how and why this cosmic urbanisation occurred. During the next few decades we shall learn a great deal more about the origin of the megacities, the nature of the first city dwellers, their organisational structure and their successes and failures.

Acknowledgments We are grateful for suggestions, useful comments and/or figures from Wil van Breugel, Ilana Feain, Jaron Kurk, Nicole Nesvadba, Roderik Overzier, Michiel Reuland, Birgitte Rocca, Huub Röttgering, Bram Venemans and Montse Villar-Martin. GM acknowledges support from an Academy Professorship of the Royal Netherlands Academy of Arts and Sciences.

Open Access This article is distributed under the terms of the Creative Commons Attribution Noncommercial License which permits any noncommercial use, distribution, and reproduction in any medium, provided the original author(s) and source are credited.

\section{Appendix: Known HZRGs}

This appendix contains a compendium of $\operatorname{HzRGs}\left(z>2, L_{500(\text { rest })}>10^{27} \mathrm{erg} \mathrm{s}^{-1} \mathrm{~Hz}^{-1}\right)$ known at the time of writing. Table 3 lists the HzRGs, their positions, redshifts, radio 
luminosities extrapolated to $500 \mathrm{MHz}$ rest frequency and relevant references. For convenience the HzRGs are listed in order of right ascencion in Table 4. A question mark indicates a value that is uncertain.

The luminosities were calculated using

$$
L_{v}=4 \pi D_{\mathrm{L}}^{2} S_{v}(1+z)^{-\alpha-1},
$$

where $D_{\mathrm{L}}$ is the luminosity distance, ${ }^{1} S_{v}$ is the observed flux density at the rest frequency, and $\alpha$ is the spectral index $\left(S_{v} \propto v^{\alpha}\right)$.

Why did we choose a rest-frame frequency of $500 \mathrm{MHz}$ to express the radio luminosities? The radio sources have steep spectra and are generally taken from low-frequency surveys, so it is appropriate to express their luminosities at as low a rest frequency as possible (e.g. Blundell and Rawlings, 2000). For consistency, surveys covering the northern and southern sky with comparable spatial resolution should be used. The most appropriate surveys are the 74 MHz VLA Low-frequency Sky Survey (VLSS; Cohen et al. 2007) and the $1.4 \mathrm{GHz}$ NVSS (Condon et al. 1998). These contain $~ 75 \%$ of the known HzRGs. For the entire redshift range of the sample, the observed frequency associated to $v_{\text {rest }}=500 \mathrm{MHz}$ is located close to the VLSS frequency, but still located between VLSS and NVSS, thereby avoiding extrapolations to un-observed parts of the radio spectrum.

For HzRGs not included in these catalogues, the following combination of surveys were used in order of preference: WENSS (Rengelink et al. 1997) and NVSS, Texas (Douglas et al. 1996) and NVSS, SUMSS (Mauch et al. 2003) and NVSS, or MRC (Large et al. 1981) and PMN (Griffith and Wright 1993). A small number of HzRGs from the CENSORS survey (Brookes et al. 2006) do not yet have measured radio spectra. For these we assumed a constant spectral index of $\alpha=-1.1$, leading to uncertainties in the derived luminosities that could be as much as $0.5 \mathrm{dex}$. The relevant luminosities are indicated by ${ }^{\mathrm{a}}$.

The table also lists references for the redshifts and relevant followup observations. Although references to the followup work are not complete, they provide a useful pointer for readers who require further information. The key to the references to followup observations are: $\mathbf{C O}=\mathrm{CO}, \mathbf{G}=$ General, $\mathbf{H}=\mathrm{HST}$ imaging, $\mathbf{H I}=\mathrm{HI}$ absorption, $\mathbf{M}=$ Millimetre/submillimetre, $\mathbf{N}=$ Nebular continuum, $\mathbf{O P}=$ Optical polarisation, $\mathbf{O S}=$ Optical/infrared spectroscopy, $\mathbf{P}=$ Protocluster, $\mathbf{R}=$ Radio, $\mathbf{S}$ Spitzer IR and $\mathbf{X}=\mathbf{X}$-rays.

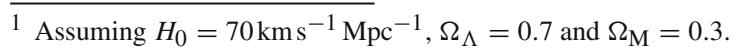


Table 3 Known radio galaxies with $z>2$ and radio luminosities $\mathrm{L}_{500(\text { rest })}>10^{27} \mathrm{~W} \mathrm{~Hz}^{-1}$

\begin{tabular}{|c|c|c|c|c|c|c|}
\hline Name & $\mathrm{RA}(\mathrm{J} 2000)$ & $\operatorname{DEC}(\mathrm{J} 2000)$ & $z$ & References & $\log \left(L_{500}\right)$ & References \\
\hline MRC 2036-254 & $20: 39: 24.5$ & $-25: 14: 31$ & 2.000 & 1 & 28.52 & $\begin{array}{l}\mathbf{R}(47,48,49,50) \\
\mathbf{X}(51)\end{array}$ \\
\hline MRC 0015-229 & $00: 17: 58.2$ & $-22: 38: 04$ & 2.01 & 1 & 28.32 & $\mathbf{R}(47,48,49,50)$ \\
\hline MRC 0156-252 & $01: 58: 33.5$ & $-24: 59: 30$ & 2.016 & 2 & 28.46 & $\begin{array}{l}\mathbf{H}(52) \mathbf{R}(47,49, \\
\quad 50) \mathbf{S}(53) \\
\quad \mathbf{X}(51)\end{array}$ \\
\hline MG 2348+0507 & $23: 48: 32.0$ & $+05: 07: 33$ & 2.019 & $\mathrm{~S} 2$ & 28.58 & \\
\hline MP J1758-6738 & $17: 58: 52.9$ & $-67: 38: 34$ & 2.026 & 3 & 28.99 & \\
\hline TXS 0448+091 & $04: 51: 14.6$ & $+09: 14: 29$ & 2.037 & 4 & 28.11 & $\mathbf{R}(47)$ \\
\hline NVSS J002402-325253 & $00: 24: 02.3$ & $-32: 52: 54$ & 2.043 & 5 & 27.93 & \\
\hline MRC 2048-272 & $20: 51: 03.4$ & $-27: 03: 05$ & 2.06 & 1 & 28.72 & $\begin{array}{l}\mathbf{H}(52) \mathbf{R}(54) \\
\mathbf{S}(53) \mathbf{X}(51)\end{array}$ \\
\hline B3 1204+401 & 12:07:06.2 & $+39: 54: 39$ & 2.066 & 6 & 28.33 & $\mathbf{R}(54)$ \\
\hline PKS 0011-023 & $00: 14: 25.5$ & $-02: 05: 56$ & 2.080 & 7 & 28.15 & $\mathbf{R}(54)$ \\
\hline $5 C 7.223$ & $08: 24: 06.4$ & $+26: 28: 12$ & 2.092 & 8 & 27.71 & \\
\hline MG 1401+0921 & $14: 01: 18.3$ & $+09: 21: 24$ & 2.093 & 9 & 28.26 & \\
\hline 7C $1740+6640$ & $17: 40: 42.2$ & $+66: 38: 44$ & 2.10 & 10 & 27.77 & \\
\hline 7C $1802+6456$ & 18:02:22.1 & $+64: 56: 45$ & 2.110 & 10 & 28.18 & \\
\hline TN J1102-1651 & 11:02:47.1 & $-16: 51: 34$ & 2.111 & 3 & 28.07 & \\
\hline $4 C+39.21$ & 07:58:08.8 & $+39: 29: 28$ & 2.119 & 11 & 28.51 & \\
\hline $6 C 1134+369$ & $11: 37: 07.7$ & $+36: 39: 54$ & 2.125 & 12 & 28.31 & $\mathbf{R}(54)$ \\
\hline TXS 2034+027 & $20: 36: 34.8$ & $+02: 56: 55$ & 2.129 & 3 & 28.45 & $\mathbf{R}(54)$ \\
\hline TXS 0214+183 & $02: 17: 25.8$ & $+18: 37: 03$ & 2.131 & 4 & 28.46 & $\mathbf{O S}(55) \mathbf{R}(47)$ \\
\hline WN J1242+3915 & $12: 42: 53.1$ & $+39: 15: 49$ & 2.131 & 3 & 28.03 & \\
\hline $4 C+40.49$ & $23: 07: 53.5$ & $+40: 41: 49$ & 2.140 & 11 & 28.46 & \\
\hline NVSS J103615-321659 & $10: 36: 15.3$ & $-32: 16: 57$ & 2.144 & 13 & 27.82 & \\
\hline $4 C+43.31$ & $13: 52: 28.5$ & $+42: 59: 23$ & 2.149 & 11 & 28.43 & \\
\hline NVSS J144932-385657 & $14: 49: 32.8$ & $-38: 56: 58$ & 2.152 & 13 & 28.07 & \\
\hline TXS 0355-037 & $03: 57: 48.0$ & $-03: 34: 08$ & 2.153 & 4 & 28.34 & OS (55) \\
\hline TNR 2254+1857 & $22: 54: 53.7$ & $+18: 57: 04$ & 2.154 & 3 & 27.80 & \\
\hline MRC 1138-262 & $11: 40: 48.3$ & $-26: 29: 10$ & 2.156 & 1 & 29.07 & $\begin{array}{l}\mathbf{G}(56) \mathbf{H}(57,52, \\
58) \\
\mathbf{M}(59,60) ; \\
\mathbf{O S}(61) ; \mathbf{P}(62, \\
63,64,65,66, \\
67,68,69) \mathbf{R} \\
(47,48,49,50) \\
\mathbf{S}(53) \\
\mathbf{X}(70,71)\end{array}$ \\
\hline $6 C * * 0746+5445$ & $07: 50: 24.6$ & $+54: 38: 07$ & 2.156 & 14 & 27.64 & \\
\hline $6 C * 0024+356$ & $00: 26: 52.0$ & $+35: 56: 24$ & 2.161 & 15 & 28.04 & \\
\hline MRC 0030-219 & $00: 33: 23.9$ & $-21: 42: 01$ & 2.168 & 2 & 28.14 & $\mathbf{R}(47,49)$ \\
\hline
\end{tabular}


Table 3 continued

\begin{tabular}{|c|c|c|c|c|c|c|}
\hline Name & $\mathrm{RA}(\mathrm{J} 2000)$ & $\mathrm{DEC}(\mathrm{J} 2000)$ & $z$ & References & $\log \left(L_{500}\right)$ & References \\
\hline B2 $1056+39$ & $10: 59: 11.5$ & $+39: 25: 01$ & 2.171 & 16 & 28.20 & $\mathbf{R}(54)$ \\
\hline $6 C * 0135+313$ & 01:38:06.6 & $+31: 32: 42$ & 2.199 & 15 & 27.97 & \\
\hline $5 \mathrm{C} 7.10$ & $08: 11: 26.5$ & $+26: 18: 19$ & 2.185 & 8 & 28.21 & \\
\hline $5 C 7.269$ & $08: 28: 39.7$ & $+25: 27: 30$ & 2.218 & 17 & 27.78 & $\mathbf{M}(21)$ \\
\hline $5 \mathrm{C} 7.271$ & $08: 28: 59.5$ & $+24: 54: 00$ & 2.224 & 8 & 27.97 & \\
\hline $6 C * 0142+427$ & $01: 45: 29.0$ & $+42: 57: 42$ & 2.225 & 15 & 28.10 & \\
\hline TXS 0200+015 & 02:02:42.9 & $+01: 49: 11$ & 2.229 & 4 & 28.07 & $\begin{array}{l}\mathbf{O A}(55,72,73) \\
\mathbf{R}(47)\end{array}$ \\
\hline NVSS J101008-383629 & 10:10:08.0 & $-38: 36: 29$ & 2.236 & 13 & 28.30 & \\
\hline TXS 1113-178 & $11: 16: 14.7$ & $-18: 06: 23$ & 2.239 & 4 & 28.46 & $\mathbf{R}(47)$ \\
\hline $6 C * 0115+394$ & 01:17:55.3 & $+39: 44: 33$ & 2.241 & 15 & 27.93 & \\
\hline $6 C 0629+53$ & $06: 33: 52.0$ & $+53: 16: 31$ & 2.246 & 17 & 28.35 & \\
\hline $6 C 0901+54$ & $09: 05: 24.2$ & $+54: 05: 42$ & 2.249 & 17 & 28.11 & \\
\hline TN J0452-1737 & $04: 52: 26.7$ & $-17: 37: 54$ & 2.26 & 3 & 28.09 & \\
\hline $4 \mathrm{C} 40.36$ & $18: 10: 55.7$ & $+40: 45: 23$ & 2.265 & 18,19 & 28.79 & $\begin{array}{l}\text { G }(74,75) ; \\
\quad \mathbf{M}(21) \\
\text { OP }(76) ; \mathbf{O S} \\
(77,78,79) \mathbf{R} \\
(47,80) ; \mathbf{S}(53)\end{array}$ \\
\hline $6 C * 0152+463$ & $01: 55: 45.6$ & $+46: 37: 11$ & 2.279 & 15 & 27.97 & \\
\hline $4 \mathrm{C} 40.22$ & $08: 59: 59.6$ & $+40: 24: 36$ & 2.28 & 20 & 28.39 & \\
\hline MRC 1324-262 & $13: 26: 54.7$ & $-26: 31: 43$ & 2.28 & 1 & 28.46 & $\mathbf{R}(47,49,50)$ \\
\hline MG 1747+1821 & $17: 47: 07.0$ & $+18: 21: 11$ & 2.281 & 21 & 28.86 & $\begin{array}{l}\mathbf{M}(21) \mathbf{O S}(77) \\
\mathbf{R}(47,54)\end{array}$ \\
\hline $6 C * 0106+397$ & 01:09:25.4 & $+40: 00: 01$ & 2.284 & 15 & 28.11 & \\
\hline MP J0340-6507 & 03:40:44.6 & $-65: 07: 12$ & 2.289 & 22 & 28.82 & \\
\hline $6 C 1106+380$ & 11:09:28.9 & $+37: 44: 31$ & 2.290 & 17 & 28.44 & \\
\hline MG 0936+0503 & 09:36:10.1 & $+05: 03: 49$ & $2.306 ?$ & $\mathrm{~S} 2$ & 28.08 & \\
\hline MG 1251+1104 & 12:51:00.1 & $+11: 04: 20$ & 2.322 & 9 & 28.42 & $\mathbf{M}(21)$ \\
\hline MRC 0349-211 & $03: 51: 11.8$ & $-20: 58: 00$ & 2.329 & 23 & 28.28 & $\mathbf{R}(48,49,50)$ \\
\hline \multirow[t]{3}{*}{ TXS 0211-122 } & $02: 14: 17.4$ & $-11: 58: 47$ & 2.340 & 24,19 & 28.48 & $\begin{array}{l}\mathbf{G}(24) \mathbf{H}(81, \\
52)\end{array}$ \\
\hline & & & & & & $\begin{array}{c}\mathbf{O P}(70) \quad \mathbf{O S} \\
(55,78,79,82)\end{array}$ \\
\hline & & & & & & $\mathbf{R}(47)$ \\
\hline \multirow[t]{2}{*}{ 4C 48.48} & 19:33:05.6 & $+48: 11: 46$ & 2.343 & 25 & 28.29 & $\mathbf{G}(24) \mathbf{M}(21)$ \\
\hline & & & & & & $\begin{array}{c}\text { OP }(76) \mathbf{O S}(78 \\
79,82) \mathbf{R}(47)\end{array}$ \\
\hline \multirow[t]{3}{*}{ TXS 1707+105 } & 17:10:06.9 & $+10: 31: 09$ & 2.349 & 4 & 28.63 & $\mathbf{H}(81,52)$ \\
\hline & & & & & & OS $(55,83)$ \\
\hline & & & & & & $\mathbf{S}(53)$ \\
\hline BRL 0128-264 & 01:30:27.9 & $-26: 09: 57$ & 2.348 & 26 & 29.13 & \\
\hline $6 C * 0118+486$ & $01: 21: 16.3$ & $+48: 57: 40$ & 2.350 & 15 & 27.83 & \\
\hline
\end{tabular}


Table 3 continued

\begin{tabular}{|c|c|c|c|c|c|c|}
\hline Name & RA(J2000) & $\operatorname{DEC}(\mathrm{J} 2000)$ & $z$ & References & $\log \left(L_{500}\right)$ & References \\
\hline NVSS J094949-213432 & $09: 49: 49.0$ & $-21: 34: 33$ & 2.354 & 27 & $26.91^{\mathrm{a}}$ & \\
\hline PKS 1425-148 & $14: 28: 41.7$ & $-15: 02: 28$ & 2.355 & 3 & 28.66 & $\mathbf{R}(54)$ \\
\hline NVSS J232651-370909 & $23: 26: 51.5$ & $-37: 09: 11$ & 2.357 & 5 & 28.02 & \\
\hline $4 \mathrm{C}-00.54$ & $14: 13: 15.1$ & $-00: 23: 00$ & 2.360 & 4,19 & 28.41 & $\begin{array}{c}\mathbf{H}(81,52) \mathbf{O P} \\
(83,76) \mathbf{O S}(55, \\
78,79,82) \mathbf{P} \\
(84) \mathbf{R}(47) \mathbf{S}(53)\end{array}$ \\
\hline MG 0001+0846 & 00:01:15.5 & $+08: 46: 39$ & $2.36 ?$ & $\mathrm{~S} 2$ & 28.53 & \\
\hline B2 1159+39A & $12: 01: 50.0$ & $+39: 19: 11$ & 2.370 & 11 & 28.22 & \\
\hline $6 C^{* *} 0854+3500$ & $08: 57: 15.9$ & $+34: 48: 24$ & 2.382 & 14 & 27.92 & \\
\hline LBDS 53W002 & $17: 14: 14.8$ & $+50: 15: 30$ & 2.390 & 28 & 27.78 & $\begin{array}{c}\mathbf{C O}(85,86) \mathbf{M}(21) \\
\mathbf{O S}(77) \mathbf{S}(53)\end{array}$ \\
\hline $6 C 0930+389$ & 09:33:06.9 & $+38: 41: 48$ & 2.395 & 17 & 28.41 & $\underset{(53)}{\mathbf{M}(21) \mathbf{R}(54) \mathbf{S}}$ \\
\hline $4 C 34.34$ & $11: 16: 30.4$ & $+34: 42: 24$ & 2.400 & 17 & 28.46 & $\mathbf{M}(21)$ \\
\hline $7 C 1736+650$ & $17: 36: 37.5$ & $+65: 02: 28$ & 2.400 & 10 & 27.65 & \\
\hline TXS 0748+134 & 07:51:01.1 & $+13: 19: 27$ & 2.419 & 4 & 28.31 & OS (55) R (47) \\
\hline NVSS J095226-200105 & $09: 52: 26.5$ & $-20: 01: 05$ & 2.421 & 27 & $26.97^{\mathrm{a}}$ & \\
\hline TN J1033-1339 & $10: 33: 10.7$ & $-13: 39: 52$ & 2.425 & 3 & 28.35 & \\
\hline NVSS J094925-203724 & $09: 49: 25.9$ & $-20: 37: 24$ & 2.427 & 27 & $27.27^{\mathrm{a}}$ & \\
\hline $4 \mathrm{C} 40.02$ & $00: 30: 49.0$ & $+41: 10: 49$ & 2.428 & 17 & 28.64 & \\
\hline B3 $0731+438$ & $07: 35: 21.9$ & $+43: 44: 21$ & 2.429 & 29 & 28.89 & $\begin{array}{c}\text { OP }(76) \text { OS }(77, \\
\quad 78,79,82) \mathbf{R}(47)\end{array}$ \\
\hline MRC 1106-258 & 11:08:30.3 & $-26: 05: 06$ & 2.43 & 1 & 28.48 & $\mathbf{R}(47,48,49,50)$ \\
\hline $5 \mathrm{C} 7.15$ & $08: 12: 19.2$ & $+26: 30: 18$ & 2.433 & 8 & 28.12 & \\
\hline MRC 0406-244 & 04:08:51.4 & $-24: 18: 17$ & 2.44 & 23 & 29.03 & $\begin{array}{l}\mathbf{H}(52) \mathbf{O S}(77) \mathbf{P} \\
\quad(84) \mathbf{R}(47,48, \\
49,50) \mathbf{S}(53) \mathbf{X} \\
(51)\end{array}$ \\
\hline $6 C * * 0834+4129$ & $08: 37: 49.2$ & $+41: 19: 54$ & 2.442 & 14 & 27.80 & \\
\hline $8 C 1536+620$ & $15: 37: 09.5$ & $+61: 55: 36$ & 2.450 & LR & 28.25 & \\
\hline MG 2308+0336 & $23: 08: 25.0$ & $+03: 37: 04$ & 2.457 & 9 & 28.51 & $\mathbf{M}(21)$ \\
\hline 4C 12.32 & $08: 55: 21.4$ & $+12: 17: 27$ & 2.468 & 30 & 28.56 & \\
\hline $3 \mathrm{C} 257$ & $11: 23: 09.4$ & $+05: 30: 18$ & 2.474 & 31 & 29.16 & $\begin{array}{l}\mathbf{M}(21) \mathbf{O S}(77) \mathbf{S} \\
(53)\end{array}$ \\
\hline \multirow[t]{4}{*}{ 4C 23.56} & 21:07:14.8 & $+23: 31: 45$ & 2.479 & 25,17 & 28.93 & $\mathbf{C O}(87) \mathbf{G}(75)$ \\
\hline & & & & & & $\begin{array}{l}\text { M (21) OP }(83, \\
76,89) \text { OS }(78, \\
82)\end{array}$ \\
\hline & & & & & & $\mathbf{P}(85) \mathbf{R}(47) \mathbf{S}(53)$ \\
\hline & & & & & & $\mathbf{X}(90)$ \\
\hline TXS $2332+154$ & $23: 34: 58.0$ & $+15: 45: 50$ & 2.481 & 3 & 28.36 & \\
\hline
\end{tabular}


Table 3 continued

\begin{tabular}{|c|c|c|c|c|c|c|}
\hline Name & RA(J2000) & $\operatorname{DEC}(\mathrm{J} 2000)$ & $z$ & References & $\log \left(L_{500}\right)$ & References \\
\hline MRC 2104-242 & 21:06:58.2 & $-24: 05: 11$ & 2.491 & 2 & 28.84 & $\begin{array}{l}\mathbf{H}(81,52) \\
\mathbf{O S}(78,91,79, \\
82) \\
\mathbf{P}(85) \mathbf{R}(48,49, \\
50,54) \mathbf{S}(53)\end{array}$ \\
\hline WN J0303+3733 & $03: 03: 26.0$ & $+37: 33: 42$ & 2.505 & 3 & 28.44 & \\
\hline TXS $1647+100$ & 16:50:05.0 & $+09: 55: 11$ & 2.509 & 3 & 28.53 & $\mathbf{R}(54)$ \\
\hline NVSS J213510-333703 & $21: 35: 10.5$ & $-33: 37: 04$ & 2.518 & 5 & 27.90 & \\
\hline TXS 1558-003 & $16: 01: 17.4$ & $-00: 28: 46$ & 2.520 & 4 & 28.82 & $\begin{array}{l}\text { OS }(55,78, \\
\quad 79,82,91) \\
\mathbf{P}(84,68) \\
\mathbf{R}(54) \mathbf{S}(53)\end{array}$ \\
\hline $8 \mathrm{C} 1039+681$ & $10: 42: 37.4$ & $+67: 50: 24$ & 2.530 & 21 & 28.56 & $\mathbf{M}(21) \mathbf{R}(54)$ \\
\hline NVSS J231144-362215 & $23: 11: 45.2$ & $-36: 22: 15$ & 2.531 & 5 & 27.83 & \\
\hline $6 C^{*} 0112+372$ & $01: 14: 50.2$ & $+37: 32: 33$ & 2.535 & 15 & 28.22 & \\
\hline TXS 1436+157 & $14: 39: 04.9$ & $+15: 31: 19$ & 2.538 & 4 & 28.24 & OS (55) R (47) \\
\hline WN J1115+5016 & $11: 15: 06.9$ & $+50: 16: 24$ & 2.54 & 3 & 27.82 & $\mathbf{M}(60) \mathbf{S}(53)$ \\
\hline MRC 2139-292 & $21: 42: 16.7$ & $-28: 58: 38$ & 2.55 & 1 & 28.73 & $\mathbf{R}(47,49,50)$ \\
\hline MP J1755-6916 & $17: 55: 29.8$ & $-69: 16: 54$ & 2.551 & 3 & 28.95 & OA (42) \\
\hline TXS 2319+223 & $23: 21: 42.4$ & $+22: 37: 55$ & 2.554 & 3 & 28.46 & $\mathbf{R}(54)$ \\
\hline $6 C * * 1045+4459$ & $10: 48: 32.4$ & $+44: 44: 28$ & 2.571 & 14 & 28.05 & \\
\hline TXS 0828+193 & 08:30:53.4 & $+19: 13: 16$ & 2.572 & 4,19 & 28.44 & $\begin{array}{l}\mathbf{H}(81) \\
\mathbf{O P}(92,76,88) \\
\mathbf{O S}(55,93,78, \\
\quad 79,82) \\
\mathbf{R}(47) \mathbf{S}(53) \\
\mathbf{X}(51)\end{array}$ \\
\hline PKS 0529-549 & $05: 30: 25.4$ & $-54: 54: 21$ & 2.575 & 4,32 & 29.16 & $\begin{array}{l}\text { OS } 55 \text { R (44) } \\
\quad \text { S }(53)\end{array}$ \\
\hline TXS 2353-003 & $23: 55: 37.0$ & $-00: 02: 58$ & 2.592 & 3 & 28.76 & \\
\hline WNH 1736+6504 & $17: 36: 37.4$ & $+65: 02: 35$ & 2.6 & 33 & 27.74 & \\
\hline MRC 2052-253 & $20: 55: 01.5$ & $-25: 09: 53$ & 2.60 & 1 & 28.77 & $\mathbf{R}(54)$ \\
\hline WN J0040+3857 & $00: 40: 56.2$ & $+38: 57: 30$ & 2.606 & 3 & 27.80 & \\
\hline 4C 26.38 & $12: 32: 23.6$ & $+26: 04: 07$ & 2.609 & 25 & 28.41 & $\mathbf{G}(75)$ \\
\hline B3 0811+391 & $08: 14: 30.6$ & $+38: 58: 35$ & 2.621 & 34 & 27.84 & \\
\hline PKS 0742+10 & $07: 45: 33.0$ & $+10: 11: 13$ & 2.624 & 35 & 28.74 & \\
\hline MRC 2025-218 & $20: 27: 59.5$ & $-21: 40: 57$ & 2.63 & 35 & 28.74 & $\begin{array}{l}\mathbf{H}(81,52) \\
\mathbf{O S}(91) \mathbf{R}(47, \\
\quad 48,49,50) \mathbf{S} \\
\quad(53)\end{array}$ \\
\hline $6 C^{*} 0122+426$ & $01: 25: 52.8$ & $+42: 51: 51$ & 2.635 & 15 & 28.04 & \\
\hline
\end{tabular}


Table 3 continued

\begin{tabular}{|c|c|c|c|c|c|c|}
\hline Name & RA(J2000) & $\operatorname{DEC}(\mathrm{J} 2000)$ & $z$ & References & $\log \left(L_{500}\right)$ & References \\
\hline MRC 0140-257 & $01: 42: 41.2$ & $-25: 30: 34$ & 2.64 & 1 & 28.44 & $\begin{array}{l}\mathbf{H}(52) \mathbf{O S}(91) \\
\mathbf{R}(47,48,49, \\
\quad 50) \mathbf{S}\end{array}$ \\
\hline TN J1941-1952 & 19:41:00.1 & $-19: 52: 14$ & 2.667 & 22 & 28.85 & \\
\hline PKS $1357+007$ & 14:00:21.4 & $+00: 30: 19$ & 2.671 & 4 & 28.72 & $\mathbf{O S}(55) \mathbf{R}(54)$ \\
\hline NVSS J233034-330009 & $23: 30: 34.5$ & $-33: 00: 12$ & 2.675 & 13 & 28.23 & \\
\hline 7C $1758+6719$ & $17: 58: 47.2$ & $+67: 19: 46$ & 2.70 & 10 & 27.99 & \\
\hline TXS 2202+128 & $22: 05: 14.3$ & $+13: 05: 34$ & 2.704 & 4 & 28.54 & $\begin{array}{l}\mathbf{H}(52) \mathbf{O A}(42) \\
\text { OS (55) R (47) } \\
\mathbf{S}(53)\end{array}$ \\
\hline NVSS J094925-200518 & $09: 49: 25.8$ & $-20: 05: 18$ & 2.706 & 27 & $27.25^{\mathrm{a}}$ & \\
\hline $6 C * * 1102+4329$ & $11: 05: 43.1$ & $+43: 13: 25$ & 2.734 & 14 & 28.17 & \\
\hline TXS 1545-234 & $15: 48: 17.5$ & $-23: 37: 02$ & 2.754 & 4 & 28.74 & $\begin{array}{l}\text { OA } \\
\quad \text { OS } \\
\mathbf{R}(47)\end{array}$ \\
\hline NVSS J152435-352623 & $15: 24: 35.4$ & $-35: 26: 22$ & 2.760 & 13 & 28.48 & \\
\hline TN J0920-0712 & $09: 20: 22.3$ & $-07: 12: 18$ & 2.762 & 3 & 28.48 & \\
\hline MG 1019+0534 & $10: 19: 33.3$ & $+05: 34: 35$ & 2.765 & 9 & 28.57 & $\begin{array}{l}\mathbf{M}(21) \mathbf{R}(54) \\
\quad \mathbf{S}(53)\end{array}$ \\
\hline NVSS J111921-363139 & $11: 19: 21.8$ & $-36: 31: 39$ & 2.769 & 13 & 29.39 & \\
\hline TXS 0417-181 & 04:19:43.6 & $-18: 01: 56$ & 2.770 & 4 & 28.64 & $\mathbf{R}(47) \mathbf{O S}(55)$ \\
\hline NVSS J213637-340318 & $21: 36: 38.4$ & $-34: 03: 21$ & 2.770 & 13 & 28.39 & \\
\hline WNR J1338+3532 & $13: 38: 15.1$ & $+35: 32: 04$ & 2.772 & 3 & 28.42 & $\mathbf{R}(54)$ \\
\hline 7C $1807+6719$ & $18: 07: 13.4$ & $+67: 19: 42$ & 2.78 & 10 & 27.99 & \\
\hline $4 \mathrm{C}+44.02$ & $00: 36: 53.5$ & $+44: 43: 21$ & 2.790 & 11 & 28.98 & \\
\hline NVSS J140223-363539 & $14: 02: 23.6$ & $-36: 35: 42$ & 2.804 & 13 & 28.34 & \\
\hline WNH 1821+6117 & $18: 22: 18.8$ & $+61: 18: 37$ & 2.81 & 33 & 27.94 & \\
\hline $4 C+03.16$ & 09:00:14.3 & $+03: 24: 08$ & 2.814 & 4 & 28.48 & \\
\hline $6 C * * 0824+5344$ & $08: 27: 58.9$ & $+53: 34: 15$ & 2.824 & 14 & 28.27 & \\
\hline NVSS J094552-201441 & $09: 45: 51.0$ & $-20: 14: 47$ & 2.829 & 27 & $27.12^{\mathrm{a}}$ & \\
\hline NVSS J095357-203652 & $09: 53: 57.4$ & $-20: 36: 51$ & 2.829 & 27 & $27.18^{\mathrm{a}}$ & \\
\hline WNH 1717+6827 & $17: 16: 56.4$ & $+68: 24: 02$ & 2.84 & 33 & 27.98 & \\
\hline MG 0148+1028 & $01: 48: 28.9$ & $+10: 28: 21$ & 2.845 & 9 & 28.56 & \\
\hline MRC 0052-241 & $00: 54: 29.8$ & $-23: 51: 30$ & 2.86 & 1 & 28.77 & $\mathbf{P}(69)$ \\
\hline B2 $1132+37$ & $11: 35: 05.9$ & $+37: 08: 41$ & 2.88 & 17 & 28.71 & $\mathbf{R}(54)$ \\
\hline $4 C 24.28$ & $13: 48: 14.8$ & $+24: 15: 50$ & 2.889 & 25 & 29.05 & $\begin{array}{c}\mathbf{G}(75) \mathbf{H}(81) \\
\mathbf{M}(21) \mathbf{R}(47, \\
\quad 80) \mathbf{S}(53)\end{array}$ \\
\hline TXS 0134+251 & 01:37:07.0 & $+25: 21: 19$ & 2.897 & 3 & 28.57 & \\
\hline NVSS J213238-335318 & $21: 32: 39.0$ & $-33: 53: 19$ & 2.900 & 13 & 28.60 & \\
\hline $4 C 28.58$ & $23: 51: 59.1$ & $+29: 10: 29$ & 2.905 & 25 & 28.91 & $\begin{array}{l}\mathbf{G}(75) \mathbf{R}(80) \\
\quad \mathbf{S}(53)\end{array}$ \\
\hline
\end{tabular}


Table 3 continued

\begin{tabular}{|c|c|c|c|c|c|c|}
\hline Name & RA(J2000) & DEC(J2000) & $z$ & References & $\log \left(L_{500}\right)$ & References \\
\hline MRC 0943-242 & $09: 45: 32.8$ & $-24: 28: 50$ & 2.922 & 1,19 & 28.62 & $\begin{array}{l}\mathbf{H}(81,52) \\
\text { OA }(95,72,73) \\
\text { OP }(76) \mathbf{O S}(78, \\
79,83) \mathbf{P}(69, \\
68) \mathbf{R}(47,49) \\
\mathbf{S}(53)\end{array}$ \\
\hline B3 $0744+464$ & $07: 47: 43.7$ & $+46: 18: 58$ & 2.926 & 29 & 28.87 & R (47) OS (77) \\
\hline NVSS J151020-352803 & $15: 10: 20.8$ & $-35: 28: 03$ & 2.937 & 13 & 28.35 & \\
\hline TXS 0952-217 & $09: 54: 29.5$ & $-21: 56: 53$ & 2.950 & 27 & $28.08^{\mathrm{a}}$ & \\
\hline WNH 1758+5821 & $17: 58: 48.0$ & $+58: 21: 41$ & 2.96 & 33 & 27.98 & \\
\hline $6 C * 0020+440$ & $00: 23: 08.5$ & $+44: 48: 23$ & 2.988 & 15 & 28.49 & \\
\hline WN J0747+3654 & $07: 47: 29.4$ & $+36: 54: 38$ & 2.992 & 3 & 28.14 & $\mathbf{M}(60) \mathbf{S}(53)$ \\
\hline $6 C^{* *} 0743+5019$ & 07:58:06.0 & $+50: 11: 03$ & 2.996 & 14 & 28.41 & \\
\hline WN J0231+3600 & $02: 31: 11.5$ & $+36: 00: 27$ & 3.080 & 3 & 28.20 & $\mathbf{M}(60)$ \\
\hline WN J1053+5424 & $10: 53: 36.3$ & $+54: 24: 42$ & 3.083 & 22 & 28.55 & \\
\hline B3 J2330+3927 & $23: 30: 24.9$ & $+39: 27: 11$ & 3.086 & 36 & 28.33 & $\begin{array}{l}\text { CO (36) HI (36) } \\
\mathbf{M}(59,60) \\
\mathbf{R}(96) \mathbf{S}(53)\end{array}$ \\
\hline TN J1112-2948 & $11: 12: 23.9$ & $-29: 48: 06$ & 3.09 & 3 & 28.76 & $\mathbf{M}(60)$ \\
\hline NVSS J095751-213321 & $09: 57: 51.3$ & $-21: 33: 21$ & 3.126 & 27 & $28.11^{\mathrm{a}}$ & \\
\hline MRC 0316-257 & $03: 18: 12.1$ & $-25: 35: 10$ & 3.142 & 2 & 28.95 & 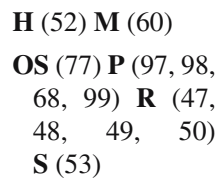 \\
\hline WN J0617+5012 & $06: 17: 39.4$ & $+50: 12: 55$ & 3.153 & 3 & 28.02 & $\mathbf{M}(60) \mathbf{S}(53)$ \\
\hline MRC 0251-273 & $02: 53: 16.7$ & $-27: 09: 10$ & 3.16 & 1 & 28.54 & $\mathbf{M}(60) \mathbf{S}(53)$ \\
\hline WN J1123+3141 & $11: 23: 55.9$ & $+31: 41: 26$ & 3.216 & 3 & 28.51 & $\mathbf{M}(60) \mathbf{S}(53)$ \\
\hline NVSS J230123-364656 & $23: 01: 23.5$ & $-36: 46: 56$ & 3.220 & 5 & 28.34 & $\mathbf{M}(60)$ \\
\hline $6 \mathrm{CE} 1232+3942$ & $12: 35: 04.8$ & $+39: 25: 39$ & 3.221 & 12 & 28.99 & $\begin{array}{l}\mathbf{M}(100,21) \\
\mathbf{R}(47) \text { OS (77) } \\
\quad \mathbf{S}(53)\end{array}$ \\
\hline WNH $1702+6042$ & $17: 03: 36.2$ & $+60: 38: 52$ & 3.223 & 33 & 28.23 & $\mathbf{M}(60)$ \\
\hline $\begin{array}{l}\text { NVSS J232100-360223 } \\
6 C^{* *} 0832+5443\end{array}$ & $\begin{array}{l}23: 21: 00.6 \\
08: 36: 09.9\end{array}$ & $\begin{array}{l}-36: 02: 25 \\
+54: 33: 26\end{array}$ & $\begin{array}{l}3.320 \\
3.341\end{array}$ & $\begin{array}{l}5 \\
14\end{array}$ & $\begin{array}{l}28.22 \\
28.24\end{array}$ & \\
\hline B2 $0902+34$ & 09:05:30.1 & $+34: 07: 57$ & 3.395 & 37 & 28.78 & $\begin{array}{c}\text { H }(81) \text { HI }(101, \\
102,103,104, \\
105) \mathbf{M}(106, \\
21) \mathbf{O S}(107,77, \\
108) \mathbf{S}(53)\end{array}$ \\
\hline NVSS J094724-210505 & $09: 47: 24.5$ & $-21: 05: 06$ & 3.377 & 27 & $27.43^{\mathrm{a}}$ & \\
\hline NVSS J095438-210425 & $09: 54: 38.4$ & $-21: 04: 25$ & 3.431 & 27 & $28.13^{\mathrm{a}}$ & \\
\hline NVSS J231402-372925 & $23: 14: 02.4$ & $-37: 29: 27$ & 3.450 & 5 & 28.83 & \\
\hline TN J0205+2242 & 02:05:10.7 & $+22: 42: 50$ & 3.506 & 3 & 28.46 & $\mathbf{M}(60) \mathbf{S}(53)$ \\
\hline
\end{tabular}


Table 3 continued

\begin{tabular}{|c|c|c|c|c|c|c|}
\hline Name & RA(J2000) & $\operatorname{DEC}(\mathrm{J} 2000)$ & $z$ & References & $\log \left(L_{500}\right)$ & References \\
\hline TN J0121+1320 & $01: 21: 42.7$ & $+13: 20: 58$ & 3.517 & 3 & 28.49 & $\begin{array}{r}\mathbf{C O}(109) \mathbf{M}(60) \\
\mathbf{O A}(110) \mathbf{S}(53)\end{array}$ \\
\hline $6 C 1909+72$ & 19:08:23.7 & $+72: 20: 12$ & 3.537 & 3 & 29.12 & $\begin{array}{l}\mathbf{C O}(111) \\
\quad \mathbf{M}(112,113) \\
\mathbf{R}(114) \mathbf{S}(53)\end{array}$ \\
\hline 4C $1243+036$ & $12: 45: 38.4$ & $+03: 23: 20$ & 3.560 & 38,19 & 29.23 & $\begin{array}{l}\mathbf{H}(81) \mathbf{M}(21) \\
\mathbf{O P}(115) \\
\mathbf{O S}(116,117) \\
\mathbf{S}(53)\end{array}$ \\
\hline WN J1911+6342 & $19: 11: 49.5$ & $+63: 42: 10$ & 3.590 & 3 & 28.14 & $\mathbf{M}(60) \mathbf{S}(53)$ \\
\hline MG 2141+192 & $21: 44: 07.5$ & $+19: 29: 15$ & 3.592 & 9 & 29.08 & $\begin{array}{l}\mathbf{H}(81) \mathbf{M}(118 \\
263) \\
\mathbf{R}(47) \mathbf{S}(53)\end{array}$ \\
\hline $6 C * 0032+412$ & $00: 34: 53.1$ & $+41: 31: 32$ & 3.658 & 15 & 28.75 & $\mathbf{M}(5) \mathbf{S}(119)$ \\
\hline TN J1049-1258 & 10:49:06.2 & $-12: 58: 19$ & 3.697 & 22 & 28.94 & \\
\hline $4 \mathrm{C} 60.07$ & $05: 12: 54.8$ & $+60: 30: 51$ & 3.791 & 25 & 29.20 & $\begin{array}{l}\mathbf{C O}(237,120) \\
\mathbf{G}(121) \mathbf{M}(5, \\
299,263) \\
\text { OS }(264) \mathbf{S}(53)\end{array}$ \\
\hline 4C 41.17 & $06: 50: 52.2$ & $+41: 30: 31$ & 3.792 & 39,40 & 29.18 & $\begin{array}{l}\mathbf{C O}(122,123 \\
124,125) \mathbf{G} \\
(126,50) \\
\mathbf{H}(127) \mathbf{M}(128, \\
54,21,299) \\
\mathbf{N}(129) \mathbf{O S}(77, \\
130,131) \\
\mathbf{P}(132,133) \\
\mathbf{R}(47,134) \\
\mathbf{S}(53) \mathbf{X}(135)\end{array}$ \\
\hline TN J2007-1316 & 20:07:53.2 & $-13: 16: 44$ & 3.837 & 22 & 29.13 & $\mathbf{M}(60) \mathbf{S}(53)$ \\
\hline NVSS J231727-352606 & $23: 17: 27.4$ & $-35: 26: 07$ & 3.874 & 5 & 28.71 & \\
\hline NVSS J021308-322338 & 02:13:08.0 & $-32: 23: 40$ & 3.976 & 5 & 28.40 & \\
\hline TN J1338-1942 & $13: 38: 26.1$ & $-19: 42: 30$ & 4.105 & 41,42 & 28.70 & $\begin{array}{l}\mathbf{M}(60) \mathbf{O A}(42) \\
\mathbf{O S}(136) \mathbf{P}(137, \\
138,139,140, \\
141,142) \\
\mathbf{R}(246) \\
\mathbf{S}(53)\end{array}$ \\
\hline TN J1123-2154 & $11: 23: 10.2$ & $-21: 54: 05$ & 4.109 & 3 & 28.45 & $\mathbf{M}(60)$ \\
\hline $8 C 1435+63$ & $14: 36: 39.0$ & $+63: 19: 04$ & 4.251 & 43 & 29.40 & $\begin{array}{c}\mathbf{G}(143) \mathbf{M}(21, \\
144,299) \\
\mathbf{R}(47) \mathbf{S}(53)\end{array}$ \\
\hline $6 \mathrm{C} 0140+326$ & $01: 43: 43.8$ & $+32: 53: 49$ & 4.413 & 44,3 & 28.73 & $\mathbf{M}(21) \mathbf{S}(53)$ \\
\hline
\end{tabular}


Table 3 continued

\begin{tabular}{lllllll}
\hline Name & RA(J2000) & DEC $(J 2000)$ & $z$ & References & Log $\left(L_{500}\right)$ & References \\
\hline RC J0311+0507 & $03: 11: 48.0$ & $+05: 08: 03$ & 4.514 & 45 & 29.49 & \\
TN J0924-2201 & $09: 24: 19.9$ & $-22: 01: 42$ & 5.197 & 46,3 & 29.51 & CO (145) M (60) \\
& & & & & & P $(146,147,141)$ \\
& & & & & S $(280)$ \\
\hline
\end{tabular}

${ }^{a}$ Radio luminosity calculated assuming a spectral index $\alpha=-1.1$

S2 Spinrad et al. MG list (private communication)

LR Lacy and Rawlings (private communication)

1 = McCarthy et al. (1996), 2 = McCarthy et al. (1990), 3 = De Breuck et al. (2001), 4 = Röttgering et al. (1997), 5 = De Breuck et al. (2006), 6 = Thompson et al. (1994), 7 = Dunlop et al. (1989), $8=$ Willott et al. (2002), $9=$ Stern et al. (1999), $10=$ Lacy et al. (1999), $11=$ Fanti et al. (2001), 12 = Rawlings et al. (1990), 13 = Bryant et al. (2007), $14=$ Cruz et al. (2006), $15=$ Jarvis et al. (2001a), $16=$ Lilly (1989), $17=$ Eales and Rawlings (1993), $18=$ Chambers et al. (1988), $19=$ Vernet et al. (2001b), $20=$ Pahre and Djorgovski (1995), 21 = Archibald et al. (2001), 22 = Bornancini et al. (2007), 23 = McCarthy (1991), 24 = van Ojik et al. (1994), 25 = Chambers et al. (1996b), 26 = Best et al. (1999), 27 = Brookes et al. (2006), 28 = Windhorst et al. (1991), 29 = McCarthy et al. (1991), 30 = Gopal-Krishna et al. (1995), $31=$ van Breugel et al. (1998), 32 = Broderick et al. (2007), 33 = Rengelink (1999), $34=$ Owen and Keel (1995), 35 = Best et al. (2003b), 36 = De Breuck et al. (2003a), 37 = Lilly (1988), 38 = van Ojik et al. (1996), 39 = Chambers et al. (1990), $40=$ Dey et al. (1997), 41 = De Breuck et al. (1999), $42=$ Wilman et al. (2004), 43 = Lacy et al. (1994), 44 = Rawlings et al. (1996), $45=$ Kopylov et al. (2006), 46 = van Breugel et al. (1999), 47 = Carilli et al. (1997), $48=$ Athreya et al. (1997), 49= Athreya and Kapahi (1998a), $50=$ Athreya et al. (1998b), $51=$ Overzier et al. (2005), $52=$ Pentericci et al. (2001), $53=$ Seymour et al. (2007), $54=$ Pentericci et al. (2000b), $55=$ van Ojik et al. (1997a), $56=$ Pentericci et al. (1997), $57=$ Pentericci et al. (1998), $58=$ Miley et al. (2006), $59=$ Stevens et al. (2003), $60=$ Reuland et al. (2004), $61=$ Nesvadba et al. (2006), 62 = Pentericci et al. (2000a), $63=$ Kurk et al. (2000), $64=$ Pentericci et al. (2002), $65=$ Kurk et al. (2004a), $66=$ Kurk et al. (2004b), $67=$ Croft et al. (2005), $68=$ Kodama et al. (2007), $69=$ Venemans et al. (2007), $70=$ Carilli et al. (1998), $71=$ Carilli et al. (2002b), $72=$ Jarvis et al. (2003), $73=$ Binette et al. (2006), $74=$ Chambers et al. (1988), $75=$ Chambers et al. (1996a), $76=$ Vernet et al. (2001b), 77 = Eales and Rawlings (1993), 78 = Villar-Martín et al. (2003), 79 = Humphrey et al. (2006), $80=$ Cai et al. (2002), $81=$ Pentericci et al. (1999), $82=$ Humphrey et al. (2007), 83 = Cimatti et al. (1998), $84=$ Kajisawa et al. (2006), $85=$ Scoville et al. (1997), $86=$ Alloin et al. (2000), $87=$ Evans et al. (1996), $88=$ Vernet et al. (2001b), 89 = Johnson et al. (2007), 90 = Villar-Martín et al. (2006), 91 = Villar-Martín et al. (2007), 92 = Knopp and Chambers (1997), 93 = Villar-Martín et al. (2002), 94 = Broderick et al. (2007), 95 = Binette et al. (2000), 96 = Pérez-Torres and De Breuck (2005), $97=$ Le Fevre et al. (1996), $98=$ Venemans et al. (2005), $99=$ Venemans et al. (2007), $100=$ Chini and Kruegel (1994), $101=$ Uson et al. (1991), 102=Briggs et al. (1993), 103=De Bruyn (1996), 104=Cody and Braun (2003), 105=Chandra et al. (2004), 106=Chini and Kruegel (1994), 107=Eales et al. (1993), 108=Reuland et al. (2007), 109=De Breuck et al. (2003b), 110=Wilman et al. (2004), 111=Papadopoulos et al. (2000), 112=Stevens et al. (2003), 113=Reuland et al. (2004), 114=Pentericci et al. (2000b), 115=Vernet et al. (2001b), 116=van Ojik et al. (1996), 117=Humphrey et al. (2007), 118=Archibald et al. (2001), 119=Seymour et al. (2007), 120=Greve et al. (2004), 121=Chambers et al. (1996a), 122=De Breuck et al. (2005), 123=Papadopoulos et al. (2005), 124=Evans et al. (1996), 125=Ivison et al. (1996), 126=Chambers et al. (1990), 127=Bicknell et al. (2000), 128=Dunlop et al. (1994), 129=Dickson et al. (1995), 130=Dey et al. (1997), 131=Reuland et al. (2007), 132=Ivison et al. (2000), 133=Greve et al. (2007), 134=Gurvits et al. (1997), 135=Scharf et al. (2003), 136=De Breuck et al. (1999), 137=Venemans et al. (2002), 138=Miley et al. (2004), 139=De Breuck et al. (2004a), 140=Intema et al. (2006), 141=Venemans et al. (2007), 142=Overzier et al. (2006a), 143=Lacy et al. (1994), 144=Stevens et al. (2003), 145=Klamer et al. (2005), 146=Venemans et al. (2004), 147=Overzier et al. (2006b) 
Table 4 HzRGs in order of right ascencion

\begin{tabular}{|c|c|c|c|c|c|}
\hline Name & $z$ & Name & $z$ & Name & $z$ \\
\hline MG 0001+0846 & 2.360 & $5 \mathrm{C} 7.271$ & 2.224 & MG 1401+0921 & 2.093 \\
\hline PKS 0011-023 & 2.080 & TXS 0828+193 & 2.572 & NVSS J140223-363539 & 2.804 \\
\hline MRC 0015-229 & 2.010 & $6 C^{* *} 0832+5443$ & 3.341 & $4 C-00.54$ & 2.360 \\
\hline $6 C * 0020+440$ & 2.988 & $6 C^{* *} 0834+4129$ & 2.442 & PKS 1425-148 & 2.355 \\
\hline NVSS J002402-325253 & 2.043 & LBDS 65W172 & 2.551 & $8 \mathrm{C} 1435+63$ & 4.251 \\
\hline $6 C * 0024+356$ & 2.161 & 4C 12.32 & 2.468 & TXS $1436+157$ & 2.538 \\
\hline $4 \mathrm{C} 40.02$ & 2.428 & $6 C^{* *} 0854+3500$ & 2.382 & NVSS J144932-385657 & 2.152 \\
\hline MRC 0030-219 & 2.168 & $4 \mathrm{C} 40.22$ & 2.280 & NVSS J151020-352803 & 2.937 \\
\hline $6 C^{*} 0032+412$ & 3.658 & $4 \mathrm{C}+03.16$ & 2.814 & NVSS J152435-352623 & 2.760 \\
\hline $4 C+44.02$ & 2.790 & $6 \mathrm{C} 0901+54$ & 2.249 & $8 \mathrm{C} 1536+620$ & 2.450 \\
\hline WN J0040+3857 & 2.606 & B2 $0902+34$ & 3.395 & TXS 1545-234 & 2.754 \\
\hline MRC 0052-241 & 2.860 & TN J0920-0712 & 2.762 & TXS 1558-003 & 2.520 \\
\hline $6 C * 0106+397$ & 2.284 & TN J0924-2201 & 5.197 & TXS $1647+100$ & 2.509 \\
\hline $6 C * 0112+372$ & 2.535 & $6 C 0930+389$ & 2.395 & WNH $1702+6042$ & 3.223 \\
\hline $6 C * 0115+394$ & 2.241 & MG 0936+0503 & 2.306 & TXS $1707+105$ & 2.349 \\
\hline $6 C * 0118+486$ & 2.350 & MRC 0943-242 & 2.922 & LBDS 53W002 & 2.390 \\
\hline TN J0121+1320 & 3.517 & NVSS J094552-201441 & 2.829 & WNH 1717+6827 & 2.840 \\
\hline $6 C * 0122+426$ & 2.635 & NVSS J094724-210505 & 3.377 & WNH 1736+6504 & 2.600 \\
\hline BRL 0128-264 & 2.348 & NVSS J094925-200518 & 2.706 & $7 C 1736+650$ & 2.400 \\
\hline TXS 0134+251 & 2.897 & NVSS J094925-203724 & 2.427 & 7C $1740+6640$ & 2.100 \\
\hline $6 C^{*} 0135+313$ & 2.199 & NVSS J094949-213432 & 2.354 & MG 1747+1821 & 2.281 \\
\hline MRC 0140-257 & 2.640 & NVSS J095226-200105 & 2.421 & MP J1755-6916 & 2.551 \\
\hline $6 C 0140+326$ & 4.413 & NVSS J095357-203652 & 2.829 & $7 C 1758+6719$ & 2.700 \\
\hline $6 C * 0142+427$ & 2.225 & TXS 0952-217 & 2.950 & WNH $1758+5821$ & 2.960 \\
\hline MG $0148+1028$ & 2.845 & NVSS J095438-210425 & 3.431 & MP J1758-6738 & 2.026 \\
\hline $6 C * 0152+463$ & 2.279 & NVSS J095751-213321 & 3.126 & 7C $1802+6456$ & 2.110 \\
\hline MRC 0156-252 & 2.016 & NVSS J101008-383629 & 2.236 & 7C $1807+6719$ & 2.780 \\
\hline TXS 0200+015 & 2.229 & MG 1019+0534 & 2.765 & $4 C 40.36$ & 2.265 \\
\hline TN J0205+2242 & 3.506 & TN J1033-1339 & 2.425 & WNH $1821+6117$ & 2.810 \\
\hline NVSS J021308-322338 & 3.976 & NVSS J103615-321659 & 2.144 & $6 C 1909+72$ & 3.537 \\
\hline TXS 0211-122 & 2.340 & $8 C 1039+681$ & 2.530 & WN J1911+6342 & 3.590 \\
\hline TXS 0214+183 & 2.131 & $6 C * * 1045+4459$ & 2.571 & $4 \mathrm{C} 48.48$ & 2.343 \\
\hline WN J0231+3600 & 3.080 & TN J1049-1258 & 3.697 & TN J1941-1952 & 2.667 \\
\hline MRC 0251-273 & 3.160 & WN J1053+5424 & 3.083 & TN J2007-1316 & 3.837 \\
\hline WN J0303+3733 & 2.505 & B2 1056+39 & 2.171 & MRC 2025-218 & 2.630 \\
\hline RC J0311+0507 & 4.514 & TN J1102-1651 & 2.111 & TXS 2034+027 & 2.129 \\
\hline MRC 0316-257 & 3.142 & $6 C^{* * * 1102+4329}$ & 2.734 & MRC 2036-254 & 2.000 \\
\hline MP J0340-6507 & 2.289 & MRC 1106-258 & 2.430 & MRC 2048-272 & 2.060 \\
\hline MRC 0349-211 & 2.329 & $6 C 1106+380$ & 2.290 & MRC 2052-253 & 2.600 \\
\hline TXS 0355-037 & 2.153 & TN J1112-2948 & 3.090 & MRC 2104-242 & 2.491 \\
\hline MRC 0406-244 & 2.440 & WN J1115+5016 & 2.540 & 4C 23.56 & 2.479 \\
\hline
\end{tabular}


Table 4 continued

\begin{tabular}{|c|c|c|c|c|c|}
\hline Name & $z$ & Name & $z$ & Name & $z$ \\
\hline TXS 0417-181 & 2.770 & TXS 1113-178 & 2.239 & NVSS J213238-335318 & 2.900 \\
\hline TXS 0448+091 & 2.037 & 4C 34.34 & 2.400 & NVSS J213510-333703 & 2.518 \\
\hline TN J0452-1737 & 2.260 & NVSS J111921-363139 & 2.769 & NVSS J213637-340318 & 2.770 \\
\hline 4C 60.07 & 3.791 & $3 \mathrm{C} 257$ & 2.474 & MRC 2139-292 & 2.550 \\
\hline PKS 0529-549 & 2.575 & TN J1123-2154 & 4.109 & MG 2141+192 & 3.592 \\
\hline WN J0617+5012 & 3.153 & WN J1123+3141 & 3.216 & TXS $2202+128$ & 2.704 \\
\hline $6 C 0629+53$ & 2.246 & B2 1132+37 & 2.880 & TNR 2254+1857 & 2.154 \\
\hline 4C 41.17 & 3.792 & $6 C 1134+369$ & 2.125 & NVSS J230123-364656 & 3.220 \\
\hline B3 $0731+438$ & 2.429 & MRC 1138-262 & 2.156 & $4 \mathrm{C}+40.49$ & 2.140 \\
\hline PKS 0742+10 & 2.624 & B2 1159+39A & 2.370 & MG 2308+0336 & 2.457 \\
\hline WN J0747+3654 & 2.992 & B3 1204+401 & 2.066 & NVSS J231144-362215 & 2.531 \\
\hline B3 0744+464 & 2.926 & 4C 26.38 & 2.609 & NVSS J231402-372925 & 3.450 \\
\hline $6 C^{* *} 0746+5445$ & 2.156 & $6 \mathrm{C} 1232+3942$ & 3.221 & NVSS J231727-352606 & 3.874 \\
\hline TXS 0748+134 & 2.419 & WN J1242+3915 & 2.131 & NVSS J232100-360223 & 3.320 \\
\hline $6 C^{* *} 0743+5019$ & 2.996 & 4C $1243+036$ & 3.560 & TXS 2319+223 & 2.554 \\
\hline $4 C+39.21$ & 2.119 & MG 1251+1104 & 2.322 & NVSS J232651-370909 & 2.357 \\
\hline $5 \mathrm{C} 7.10$ & 2.185 & MRC 1324-262 & 2.280 & B3 J2330+3927 & 3.086 \\
\hline $5 \mathrm{C} 7.15$ & 2.433 & WNR J1338+3532 & 2.772 & NVSS J233034-330009 & 2.675 \\
\hline B3 $0811+391$ & 2.621 & TN J1338-1942 & 4.105 & TXS 2332+154 & 2.481 \\
\hline $5 C 7.223$ & 2.092 & 4C 24.28 & 2.889 & MG 2348+0507 & 2.019 \\
\hline $6 C^{* *} 0824+5344$ & 2.824 & $4 C+43.31$ & 2.149 & 4C 28.58 & 2.905 \\
\hline $5 C 7.269$ & 2.218 & PKS 1357+007 & 2.671 & TXS 2353-003 & 2.592 \\
\hline
\end{tabular}

\section{References}

Allen MG, Dopita MA, Tsvetanov ZI (1998) Ultraviolet Diagnostics for the Emission-Line Gas in Active Galaxies. Astrophys J 493:571 doi:10.1086/305145, arXiv:astro-ph/9801040

Aller LH (ed) (1984) Physics of thermal gaseous nebulae. astrophysics and space science library, vol 112 Alloin D, Barvainis R, Guilloteau S (2000) New CO and millimetre continuum observations of the $Z=2.394$ radio galaxy 53W002. Astrophys J Lett 528:L81-L84. doi:10.1086/312429, arXiv:astro$\mathrm{ph} / 9910196$

Antonucci R (1993) Unified models for active galactic nuclei and quasars. ARA\& A 31:473-521. doi:10. 1146/annurev.aa.31.090193.002353

Archibald EN, Dunlop JS, Hughes DH, Rawlings S, Eales SA, Ivison RJ (2001) A submillimetre survey of the star formation history of radio galaxies. Mon Not Roy Astro Soc 323:417-444

Armus L, Heckman TM, Miley GK (1990) The optical emission-line nebulae of powerful far-infrared galaxies. Astrophys J 364:471-495. doi:10.1086/169431

Arnaboldi M, Neeser MJ, Parker LC, Rosati P, Lombardi M, Dietrich JP, Hummel W (2007) ESO Public Surveys with the VST and VISTA. The Messenger 127:28

Athreya RM, Kapahi VK (1998) The redshift dependence of spectral index in powerful radio galaxies. J Astrophys Astro 19:63-77

Athreya RM, Kapahi VK, McCarthy PJ, van Breugel W (1997) Steep- spectrum radio cores in high-redshift galaxies. Mon Not Roy Astro Soc 289:525-534

Athreya RM, Kapahi VK, McCarthy PJ, van Breugel W (1998) Large rotation measures in radio galaxies at $\mathrm{Z}>2$. Astron Astrophys 329:809-820 
Baade W, Minkowski R (1954) Identification of the radio sources in Cassiopeia, Cygnus a, and Puppis a. Astrophys J 119:206

Baugh CM, Cole S, Frenk CS, Lacey CG (1998) The epoch of galaxy formation. Astrophys J 498:504. doi:10.1086/305563, arXiv:astro-ph/9703111

Bavdaz M, Lumb D, Gondoin P, Lyngvi A, Rando N, Peacock T, van der Laan T, Wallace K, Mieremet A, Oemrawsingh S, Beijersbergen M, Collon M, Kraft S, Graue R, Kampf D, Freyberg M (2006) The XEUS X-ray telescope. In: Space Telescopes and instrumentation II: Ultraviolet to gamma ray. Edited by Turner, MJL, Hasinger G (eds) Proceedings of the SPIE. Presented at the Society of photo-optical Instrumentation engineers (SPIE) conference, vol. 62663, pp. 62661. doi:10.1117/12.670240

Begelman MC, Cioffi DF (1989) Overpressured cocoons in extragalactic radio sources. Astrophys J Lett 345:L21-L24. doi:10.1086/185542

Belsole E, Worrall DM, Hardcastle MJ, Birkinshaw M, Lawrence CR (2004) XMM-Newton observations of three high-redshift radio galaxies. Mon Not Roy Astro Soc 352:924-938. doi:10.1111/j.1365-2966. 2004.07983.x, arXiv:astro-ph/0405116

Best PN (2007) Feedback from radio-loud AGN. New Astro Rev 51:168-173. doi:10.1016/j.newar.2006. 11.014

Best PN, Longair MS, Röttgering HJA (1998) HST, radio and infrared observations of 28 3CR radio galaxies at redshift $\mathrm{z} \sim 1$. II-Old stellar populations in central cluster galaxies. Mon Not Roy Astro Soc 295:549, arXiv:astro-ph/9709195

Best PN, Röttgering HJA, Lehnert MD (1999) A 98 per cent spectroscopically complete sample of the most powerful equatorial radio sources at $408 \mathrm{MHz}$. Mon Not Roy Astro Soc 310:223-254, arXiv:astro$\mathrm{ph} / 9903471$

Best PN, Röttgering HJA, Longair MS (2000) Ionisation, shocks and evolution of the emission-line gas of distant 3CR radio galaxies. Mon Not Roy Astro Soc 311:23-36. arXiv:astro-ph/9908211

Best PN, Lehnert MD, Miley GK, Röttgering HJA (2003a) Red galaxy overdensities and the varied cluster environments of powerful radio sources with $\mathrm{z} \sim 1.6$. Mon Not Roy Astro Soc 343:1-21. doi:10.1046/ j.1365-8711.2003.06667.x, arXiv:astro-ph/0304035

Best PN, Peacock JA, Brookes MH, Dowsett RE, Röttgering HJA, Dunlop JS, Lehnert MD (2003b) The final two redshifts for radio sources from the equatorial BRL sample. Mon Not Roy Astro Soc 346:10211024. doi:10.1111/j.1365-2966.2003.07156.x, arXiv:astro-ph/0309081

Best PN, Kauffmann G, Heckman TM, Brinchmann J, Charlot S, Ivezić Ž, White SDM (2005) The host galaxies of radio-loud active galactic nuclei: mass dependences, gas cooling and active galactic nuclei feedback. Mon Not Roy Astro Soc 362:25-40. doi:10.1111/j.1365-2966.2005.09192.x, arXiv:astro$\mathrm{ph} / 0506269$

Bicknell GV, Sutherland RS, van Breugel WJM, Dopita MA, Dey A, Miley GK (2000) Jet-induced Emission-Line Nebulosity and Star Formation in the High-Redshift Radio Galaxy 4C 41.17. Astrophys J 540:678-686. doi:10.1086/309343, arXiv:astro-ph/9909218

Binette L, Kurk JD, Villar-Martín M, Röttgering HJA (2000) A vestige low metallicity gas shell surrounding the radio galaxy 0943-242 at $z=2.92$. Astron Astrophys 356:23-32. astro-ph/0002210

Binette L, Wilman RJ, Villar-Martín M, Fosbury RAE, Jarvis MJ, Röttgering HJA (2006) Ionisation of large-scale absorbing haloes and feedback events from high-redshift radio galaxies. Astron Astrophys 459:31-42. doi:10.1051/0004-6361:20065079, astro-ph/0607289

Blandford RD (2001) Black holes and relativistic jets. Prog Theor Phys Suppl 143:182-201. arXiv:astro$\mathrm{ph} / 0110394$

Blumenthal G, Miley G (1979) Spectral index dependent properties of steep spectrum radio sources. Astron Astrophys 80:13-21

Blundell KM, Rawlings S (1999) The inevitable youthfulness of known high-redshift radio galaxies. Nature 399:330-332. astro-ph/9905333

Blundell KM, Rawlings S (2000) The Spectra and Energies of Classical Double Radio Lobes. Astron J 119:1111-1122. doi:10.1086/301254, arXiv:astro-ph/0001327

Blundell KM, Rawlings S, Eales SA, Taylor GB, Bradley AD (1998) A sample of 6C radio sources designed to find objects at redshift z>4 - I. The radio data. Mon Not Roy Astro Soc 295:265-279. arXiv:astro$\mathrm{ph} / 9707336$

Blundell KM, Fabian AC, Crawford CS, Erlund MC, Celotti A (2006) Discovery of the Low-Energy Cutoff in a Powerful Giant Radio Galaxy. Astrophys J Lett 644:L13-L16. doi:10.1086/504839, arXiv:astro$\mathrm{ph} / 0605391$ 
Bornancini CG, De Breuck C, de Vries W, Croft S, van Breugel W, Röttgering H, Minniti D (2007) Imaging and spectroscopy of ultrasteep spectrum radio sources. Mon Not Roy Astro Soc 378:551-562. doi:10. 1111/j.1365-2966.2007.11769.x, arXiv:astro-ph/0703664

Briggs FH, Sorar E, Taramopoulos A (1993) Arecibo observation of B2 $0902+343$ in the 21 Centimeter Line at Redshift 3.4. Astrophys J Lett 415:L99. doi:10.1086/187042

Broderick JW, De Breuck C, Hunstead RW, Seymour N (2007) An extreme rotation measure in the high-redshift radio galaxy PKS B0529-549. Mon Not Roy Astro Soc 375:1059-1069. doi:10.1111/j. 1365-2966.2006.11375.x, arXiv:astro-ph/0612143

Brookes MH, Best PN, Rengelink R, Röttgering HJA (2006) CENSORS: a combined EIS-NVSS survey of radio sources-II. Infrared imaging and the K-z relation. Mon Not Roy Astro Soc 366:1265-1288. doi:10.1111/j.1365-2966.2005.09916.x, arXiv:astro-ph/0603655

Brown RL, Wild W, Cunningham C (2004) ALMA-the Atacama large millimetre array. Advances in Space Research 34:555-559. doi:10.1016/j.asr.2003.03.028

Bruzual AG (2007) On TP-AGB stars and the mass of galaxies. ArXiv Astrophysics e-prints astro$\mathrm{ph} / 0703052$

Bryant J, Broderick J, Johnston H, Hunstead R, Gaensler B, De Breuck C (2007) A new search for distant radio galaxies in the southern hemisphere- II. K-band imaging and optical spectroscopy. Mon Not Roy Astro Soc

Buchalter A, Helfand DJ, Becker RH, White RL (1998) Constraining Omega 0 with the Angular SizeRedshift Relation of Double-lobed Quasars in the FIRST Survey. Astrophys J 494:503. doi:10.1086/ 305236, arXiv:astro-ph/9709174

Cai Z, Nan R, Schilizzi RT, Miley GK, Bremer MAR, van Dam B, Röttgering HJA, Liang H, Chambers KC, Gurvits LI, Zhang HY (2002) A 327 MHz VLBI study of high redshift radio galaxies $1345+245$, $1809+407$ and 2349+289. Astron Astrophys 381:401-407. doi:10.1051/0004-6361:20011579

Carilli CL (2006) HI $21 \mathrm{~cm}$ probes of reionisation, and beyond. New Astro Review 50:162-172. doi:10. 1016/j.newar.2005.11.031, arXiv:astro-ph/0509055

Carilli CL, Owen FN, Harris DE (1994) Radio continuum polarimetric imaging of high redshift radio galaxies. Astron J 107:480-493. doi:10.1086/116870

Carilli CL, Röttgering HJA, van Ojik R, Miley GK, van Breugel WJM (1997) Radio Continuum Imaging of High-Redshift Radio Galaxies. Astrophys J Suppl 109:1. doi:10.1086/312973, arXiv:astro$\mathrm{ph} / 9610157$

Carilli CL, Harris DE, Pentericci L, Rottergering HJA, Miley GK, Bremer MN (1998) An X-Ray Cluster at Redshift 2.156? Astrophys J Lett 494:L143. doi:10.1086/311199, arXiv:astro-ph/9802066

Carilli CL, Gnedin NY, Owen F (2002a) H I 21 Centimeter Absorption beyond the Epoch of Reionisation. Astrophys J 577:22-30. doi:10.1086/342179, arXiv:astro-ph/0205169

Carilli CL, Harris DE, Pentericci L, Röttgering HJA, Miley GK, Kurk JD, van Breugel W (2002b) The X-Ray-Radio Alignment in the $z=2.2$ Radio Galaxy PKS 1138-262. Astrophys J 567:781-789. doi:10.1086/338669, arXiv:astro-ph/0111193

Chambers KC, Miley GK, van Breugel W (1987) Alignment of radio and optical orientations in high-redshift radio galaxies. Nature 329:604-606. doi:10.1038/329604a0

Chambers KC, Miley GK, van Breugel WJM (1988) 4C 40.36 - A radio galaxy at a redshift of 2.3. Astrophys J Lett 327:L47-L50. doi:10.1086/185137

Chambers KC, Miley GK, van Breugel WJM (1990) 4C 41.17 - A radio galaxy at a redshift of 3.8. Astrophys J 363:21-39. doi:10.1086/169316

Chambers KC, Miley GK, van Breugel WJM, Bremer MAR, Huang JS, Trentham NA (1996a) Ultra-SteepSpectrum Radio Sources. II. Radio, Infrared, Optical, and HST Imaging of High-Redshift 4C Objects. Astrophys J Suppl 106:247. doi:10.1086/192338

Chambers KC, Miley GK, van Breugel WJM, Huang JS (1996b) Ultra-Steep-Spectrum Radio Sources. I. 4C Objects. Astrophys J Suppl 106:215. doi:10.1086/192337

Chandra P, Swarup G, Kulkarni VK, Kantharia NG (2004) Associated HI Absorption in the z $=3.4$ Radio Galaxy B2 $0902+343$ Observed with the GMRT. J Astrophys Astro 25:57. arXiv:astro-ph/0407380

Chapman SC, Lewis GF, Scott D, Richards E, Borys C, Steidel CC, Adelberger KL, Shapley AE (2001) Submillimetre imaging of a protocluster region at $Z=3.09$. Astrophys J Lett 548:L17-L21. doi:10. 1086/318919, arXiv:astro-ph/0010101

Chini R, Kruegel E (1994) Dust at high Z. Astron Astrophys 288:L33-L36

Cimatti A, di Serego-Alighieri S, Fosbury RAE, Salvati M, Taylor D (1993) Optical Polarisation in Distant Radio Galaxies. Mon Not Roy Astro Soc 264:421 
Cimatti A, di Serego Alighieri S, Vernet J, Cohen M, Fosbury RAE (1998) The UV Radiation from Z approximately 2.5 Radio Galaxies: Keck Spectropolarimetry of 4C 23.56 and 4C 00.54. Astrophys J Lett 499:L21. doi:10.1086/311354, arXiv:astro-ph/9803311

Cody AM, Braun R (2003) Observations of the high-redshift galaxy B2 $0902+343$ at $92 \mathrm{~cm}$. Astron Astrophys 400:871-875. doi:10.1051/0004-6361:20030082, arXiv:astro-ph/0301418

Cohen AS, Lane WM, Cotton WD, Kassim NE, Lazio TJW, Perley RA, Condon JJ, Erickson WC (2007) The VLA low-frequency sky survey. Astron J 134:1245-1262. doi:10.1086/520719, arXiv:0706.1191

Colbert JW, Teplitz H, Francis P, Palunas P, Williger GM, Woodgate B (2006) Ultraviolet-bright, high-redshift ultraluminous infrared galaxies. Astrophys J Lett 637:L89-L92. doi:10.1086/500647, arXiv:astro-ph/0512583

Condon JJ, Cotton WD, Greisen EW, Yin QF, Perley RA, Taylor GB, Broderick JJ (1998) The NRAO VLA sky survey. Astron J 115:1693-1716. doi:10.1086/300337

Conti PS, Leitherer C, Vacca WD (1996) Hubble space telescope ultraviolet spectroscopy of NGC 1741: a nearby template for distant energetic starbursts. Astrophys J Lett 461:L87. doi:10.1086/310005, arXiv:astro-ph/9602084

Cowie LL, Songaila A, Hu EM, Cohen JG (1996) New insight on galaxy formation and evolution from Keck spectroscopy of the Hawaii deep fields. Astron J 112:839. doi:10.1086/118058, arXiv:astro$\mathrm{ph} / 9606079$

Croft S, Kurk J, van Breugel W, Stanford SA, de Vries W, Pentericci L, Röttgering H (2005) The filamentary large-scale structure around the $z=2.16$ radio galaxy PKS 1138-262. Astron J 130:867-872. doi:10. 1086/431956, arXiv:astro-ph/0505354

Croft S, van Breugel W, de Vries W, Dopita M, Martin C, Morganti R, Neff S, Oosterloo T, Schiminovich D, Stanford SA, van Gorkom J (2006) Minkowski's object: a starburst triggered by a radio jet, revisited. Astrophys J 647:1040-1055. doi:10.1086/505526, arXiv:astro-ph/0604557

Croton DJ, Springel V, White SDM, De Lucia G, Frenk CS, Gao L, Jenkins A, Kauffmann G, Navarro JF, Yoshida N (2006) The many lives of active galactic nuclei: cooling flows, black holes and the luminosities and colours of galaxies. Mon Not Roy Astro Soc 365:11-28. doi:10.1111/j.1365-2966. 2005.09675.x, arXiv:astro-ph/0508046

Cruz MJ, Jarvis MJ, Blundell KM, Rawlings S, Croft S, Klöckner HR, McLure RJ, Simpson C, Targett TA, Willott CJ (2006) The 6C** sample of steep-spectrum radio sources-I. Radio data, near-infrared imaging and optical spectroscopy. Mon Not Roy Astro Soc 373:1531-1562. doi:10.1111/j.1365-2966. 2006.11101.x, arXiv:astro-ph/0609790

Daly RA, Guerra EJ (2002) Quintessence, Cosmology, and Fanaroff-Riley Type IIb Radio Galaxies. Astron J 124:1831-1838. doi:10.1086/342741, arXiv:astro-ph/0209503

De Breuck C, Reuland M (2005) Star formation in high redshift radio galaxies. In: Bender R, Renzini A (eds) Multiwavelength mapping of galaxy formation and evolution, p 374. doi:10.1007/10995020_67

De Breuck C, van Breugel W, Minniti D, Miley G, Röttgering H, Stanford SA, Carilli C (1999) VLT spectroscopy of the $z=4.11$ Radio Galaxy TN J1338-1942. Astron Astrophys 352:L51-L56. arXiv:astro-ph/9909178

De Breuck C, Röttgering H, Miley G, van Breugel W, Best P (2000a) A statistical study of emission lines from high redshift radio galaxies. Astron Astrophys 362:519-543. arXiv:astro-ph/0008264

De Breuck C, van Breugel W, Röttgering HJA, Miley G (2000b) A sample of 669 ultra steep spectrum radio sources to find high redshift radio galaxies. Astron Astrophys Suppl 143:303-333. arXiv:astro$\mathrm{ph} / 0002297$

De Breuck C, van Breugel W, Röttgering H, Stern D, Miley G, de Vries W, Stanford SA, Kurk J, Overzier R (2001) Spectroscopy of ultra-steep-spectrum radio sources. Astron J 121:1241-1265. doi:10.1086/ 319392, arXiv:astro-ph/0012065

De Breuck C, van Breugel W, Stanford SA, Röttgering H, Miley G, Stern D (2002) Optical and near-infrared imaging of ultra-steep-spectrum radio sources: the $\mathrm{K}-\mathrm{z}$ diagram of radio-selected and optically selected galaxies. Astron J 123:637-677. doi:10.1086/324632, arXiv:astro-ph/0109540

De Breuck C, Neri R, Morganti R, Omont A, Rocca-Volmerange B, Stern D, Reuland M, van Breugel W, Röttgering H, Stanford SA, Spinrad H, Vigotti M, Wright M (2003a) CO emission and associated H I absorption from a massive gas reservoir surrounding the $z=3$ radio galaxy B3 J2330 + 3927. Astron Astrophys 401:911-925. doi:10.1051/0004-6361:20030171, arXiv:astro-ph/0302154

De Breuck C, Neri R, Omont A (2003b) CO emission from/z>3 radio galaxies. New Astro Rev 47:285-289. arXiv:astro-ph/0302196 
De Breuck C, Bertoldi F, Carilli C, Omont A, Venemans B, Röttgering H, Overzier R, Reuland M, Miley G, Ivison R, van Breugel W (2004a) A multi-wavelength study of the proto-cluster surrounding the $z=4.1$ radio galaxy TN J1338-1942. Astron Astrophys 424:1-12. doi:10.1051/0004-6361:20035885, arXiv:astro-ph/0405339

De Breuck C, Hunstead RW, Sadler EM, Rocca-Volmerange B, Klamer I (2004b) A search for distant radio galaxies from SUMSS and NVSS - I. Sample definition, radio and K-band imaging. Mon Not Roy Astro Soc 347:837-853. doi:10.1111/j.1365-2966.2004.07270.x, arXiv:astro-ph/0309814

De Breuck C, Downes D, Neri R, van Breugel W, Reuland M, Omont A, Ivison R (2005) Detection of two massive CO systems in 4C 41.17 at $z=3.8$. Astron Astrophys 430:L1-L4. doi:10.1051/0004-6361: 200400115, arXiv:astro-ph/0411732

De Breuck C, Klamer I, Johnston H, Hunstead RW, Bryant J, Rocca-Volmerange B, Sadler EM (2006) A search for distant radio galaxies from SUMSS and NVSS-II. Optical spectroscopy. Mon Not Roy Astro Soc 366:58-72. doi:10.1111/j.1365-2966.2005.09799.x, arXiv:astro-ph/0511169

De Bruyn AG (1996) H I at High Redshift. In: Bremer MN, Malcolm N (eds) Cold Gas at High Redshift, Astrophysics and Space Science Library, vol 206, pp 171

De Lucia G, Blaizot J (2007) The hierarchical formation of the brightest cluster galaxies. Mon Not Roy Astro Soc 375:2-14. doi:10.1111/j.1365-2966.2006.11287.x, arXiv:astro-ph/0606519

de Vries WH, Becker RH, White RL (2006) Long-Term Optical Quasar Variability. In: Gaskell CM, McHardy IM, Peterson BM, Sergeev SG (eds) Astronomical Society of the Pacific conference series, vol 360, p 29

De Young DS (1989) Star formation in radio galaxies at large redshift. Astrophys J Lett 342:L59-L62. doi:10.1086/185484

Dey A, van Breugel W, Vacca WD, Antonucci R (1997) Triggered star formation in a massive galaxy at $Z=3.8:$ 4C 41.17. Astrophys J 490:698. doi:10.1086/304911, astro-ph/9707166

Dey A, Bian C, Soifer BT, Brand K, Brown MJI, Chaffee FH, Le Floc'h E, Hill G, Houck JR, Jannuzi BT, Rieke M, Weedman D, Brodwin M, Eisenhardt P (2005) Discovery of a large $~ 200$ kpc gaseous nebula at $\mathrm{z} \sim 2.7$ with the Spitzer space telescope. Astrophys J 629:654-666. doi:10.1086/430775, arXiv:astro-ph/0503632

Di Matteo T, Springel V, Hernquist L (2005) Energy input from quasars regulates the growth and activity of black holes and their host galaxies. Nature 433:604-607. doi:10.1038/nature03335, arXiv:astro$\mathrm{ph} / 0502199$

di Serego Alighieri S (1997) Polarimetry with large telescopes. Rodriguez Espinosa JM, Herrero A, Sánchez F (eds) instrumentation for large telescopes, pp. 340. ISBN 0521582911. Cambridge University Press, Cambridge, pp 287-329

di Serego Alighieri S, Fosbury RAE, Tadhunter CN, Quinn PJ (1989) Polarised light in high-redshift radio galaxies. Nature 341:307-309. doi:10.1038/341307a0

Dickson R, Tadhunter C, Shaw M, Clark N, Morganti R (1995) The nebular contribution to the extended UV continua of powerful radio galaxies. Mon Not Roy Astro Soc 273:L29-L33

Dijkstra M, Haiman Z, Spaans M (2006a) Ly $\alpha$ radiation from collapsing protogalaxies. I. Characteristics of the emergent spectrum. Astrophys J 649:14-36. doi:10.1086/506243, arXiv:astro-ph/0510407

Dijkstra M, Haiman Z, Spaans M (2006b) Ly $\alpha$ radiation from collapsing protogalaxies. II. Observational evidence for gas infall. Astrophys J 649:37-47. doi:10.1086/506244, arXiv:astro-ph/0510409

Dopita MA, Sutherland RS (2003) Astrophysics of the diffuse universe. Astro and astrophysics library, Springer, Berlin ISBN 3540433627

Douglas JN, Bash FN, Bozyan FA, Torrence GW, Wolfe C (1996) The Texas survey of radio sources covering -35.5 degrees < declination < 71.5 degrees at $365 \mathrm{MHz}$. Astron J 111:1945. doi:10.1086/ 117932

Downes D, Solomon PM, Radford SJE (1993) Molecular gas mass and far-infrared emission from distant luminous galaxies. Astrophys J Lett 414:L13-L16. doi:10.1086/186984

Dubinski J (1998) The Origin of the Brightest Cluster Galaxies. Astrophys J 502:141. doi:10.1086/305901, arXiv:astro-ph/9709102

Dunlop JS, Peacock JA (1990) The Redshift Cut-Off in the Luminosity Function of Radio Galaxies and Quasars. Mon Not Roy Astro Soc 247:19

Dunlop JS, Peacock JA, Savage A, Lilly SJ, Heasley JN, Simon AJB (1989) The parkes selected regionsdeep optical and infrared observations of radio galaxies and quasars at high redshifts. Mon Not Roy Astro Soc 238:1171-1231 
Dunlop JS, Hughes DH, Rawlings S, Eales SA, Ward MJ (1994) Detection of a large mass of dust in a radio galaxy at redshift $Z=3.8$. Nature 370:347. doi:10.1038/370347a0

Eales S, Rawlings S, Puxley P, Rocca-Volmerange B, Kuntz K (1993) Evidence that the $Z=3.4$ radio galaxy B2 $0902+34$ may be a protogalaxy. Nature 363:140-142. doi:10.1038/363140a0

Eales S, Rawlings S, Law-Green D, Cotter G, Lacy M (1997) A first sample of faint radio sources with virtually complete redshifts. I-Infrared images, the Hubble diagram and the alignment effect. Mon Not Roy Astro Soc 291:593. arXiv:astro-ph/9701023

Eales SA, Rawlings S (1993) Infrared spectroscopy of 11 radio galaxies at Z equals 2-4_Evidence that some high-redshift radio galaxies may be protogalaxies. Astrophys J 411:67-88. doi:10.1086/172808

Eales SA, Rawlings S (1996) A panoramic view of radio galaxy evolution from a redshift of 0 to a redshift of 4.3. Astrophys J 460:68. doi:10.1086/176953

Eke VR, Cole S, Frenk CS (1996) Cluster evolution as a diagnostic for Omega. Mon Not Roy Astro Soc 282:263-280. arXiv:astro-ph/9601088

Elmegreen DM, Elmegreen BG, Rubin DS, Schaffer MA (2005) Galaxy morphologies in the hubble ultra deep field: dominance of linear structures at the detection limit. Astrophys J 631:85-100. doi:10.1086/ 432502, arXiv:astro-ph/0508216

Erlund MC, Fabian AC, Blundell KM, Celotti A, Crawford CS (2006) Extended inverse-compton emission from distant, powerful radio galaxies. Mon Not Roy Astro Soc 371:29-37. doi:10.1111/j.1365-2966. 2006.10660.x, arXiv:astro-ph/0606238

Evans AS, Sanders DB, Mazzarella JM, Solomon PM, Downes D, Kramer C, Radford SJE (1996) A search for $\mathrm{CO}$ emission in high-redshift powerful radio galaxies. Astrophys J 457:658. doi:10.1086/176761

Fabian AC (1994) Cooling Flows in Clusters of Galaxies. ARA\& A 32:277-318. doi:10.1146/annurev.aa. 32.090194.001425

Fabian AC, Celotti A, Johnstone RM (2003a) Chandra reveals X-rays along the radio axis in the quasar 3C 9 at $z=2.012$. Mon Not Roy Astro Soc 338:L7-L11. doi:10.1046/j.1365-8711.2003.06111.x, arXiv:astro-ph/0204488

Fabian AC, Sanders JS, Crawford CS, Ettori S (2003b) A deep Chandra observation of the cluster environment of the $z=1.786$ radio galaxy 3C 294. Mon Not Roy Astro Soc 341:729-738. doi:10.1046/j. 1365-8711.2003.06394.x, arXiv:astro-ph/0301468

Fall SM, Rees MJ (1977) Survival and disruption of galactic substructure. Mon Not Roy Astro Soc 181:37P$42 \mathrm{P}$

Fan X, Narayanan VK, Lupton RH, Strauss MA, Knapp GR, Becker RH, White RL, Pentericci L, Leggett SK, Haiman Z, Gunn JE, Ivezić Ž, Schneider DP, Anderson SF, Brinkmann J, Bahcall NA, Connolly AJ, Csabai I, Doi M, Fukugita M, Geballe T, Grebel EK, Harbeck D, Hennessy G, Lamb DQ, Miknaitis G, Munn JA, Nichol R, Okamura S, Pier JR, Prada F, Richards GT, Szalay A, York DG (2001) A survey of $z>5.8$ quasars in the sloan digital sky survey. I. Discovery of three new quasars and the spatial density of luminous quasars at $\mathrm{z} \sim 6$. Astron J 122:2833-2849. doi:10.1086/324111, arXiv:astro-ph/0108063

Fan X, Carilli CL, Keating B (2006) Observational constraints on cosmic reionisation. ARA\& A 44:415462, arXiv:astro-ph/0602375

Fanaroff BL, Riley JM (1974) The morphology of extragalactic radio sources of high and low luminosity. Mon Not Roy Astro Soc 167:31P-36P

Fanti C, Pozzi F, Dallacasa D, Fanti R, Gregorini L, Stanghellini C, Vigotti M (2001) Multi-frequency VLA observations of a new sample of CSS/GPS radio sources. Astron \& Astrophys 369:380-420. doi:10. 1051/0004-6361:20010051

Felten JE, Morrison P (1966) Omnidirectional inverse compton and synchrotron radiation from cosmic distributions of fast electrons and thermal photons. Astrophys J 146:686

Felten JE, Rees MJ (1969) Cosmological implications of the diffuse X-ray background. Nature 221:924

Fomalont EB, Miley GK (1975) Structure of the compact radio component in 3C236. Nature 257:99-103

Francis PJ, Williger GM, Collins NR, Palunas P, Malumuth EM, Woodgate BE, Teplitz HI, Smette A, Sutherland RS, Danks AC, Hill RS, Lindler D, Kimble RA, Heap SR, Hutchings JB (2001) A pair of compact red galaxies at redshift 2.38, immersed in a 100 kiloparsec scale Ly $\alpha$ nebula. Astrophys $\mathrm{J}$ 554:1001-1011. doi:10.1086/321417, arXiv:astro-ph/0102263

Fu H, Stockton A (2007) A common origin for quasar extended emission-line regions and their broad-line regions. Astrophys J Lett 664:L75-L78. doi:10.1086/520959, arXiv:0706.2941

Furlanetto SR, Loeb A (2002) The 21 centimeter forest: radio absorption spectra as probes of minihalos before reionisation. Astrophys J 579:1-9. doi:10.1086/342757, arXiv:astro-ph/0206308 
Fynbo JU, Møller P, Warren SJ (1999) Extended LY alpha emission from a damped LY alpha absorber at $z=1.93$, and the relation between damped LY alpha absorbers and Lyman-break galaxies. Mon Not Roy Astro Soc 305:849-858, arXiv:astro-ph/9812434

Gao L, Loeb A, Peebles PJE, White SDM, Jenkins A (2004) Early formation and late merging of the giant galaxies. Astrophys J 614:17-25. doi:10.1086/423444, arXiv:astro-ph/0312499

Gardner JP, Mather JC, Clampin M, Doyon R, Greenhouse MA, Hammel HB, Hutchings JB, Jakobsen P, Lilly SJ, Long KS, Lunine JI, McCaughrean MJ, Mountain M, Nella J, Rieke GH, Rieke MJ, Rix HW, Smith EP, Sonneborn G, Stiavelli M, Stockman HS, Windhorst RA, Wright GS (2006) The James Webb space telescope. Space Sci Rev 123:485-606. doi:10.1007/s11214-006-8315-7, arXiv:astro$\mathrm{ph} / 0606175$

Ge J, Owen FN (1994) Faraday rotation in cooling flow clusters of galaxies. 2: Survey. Astron J 108:15231533. doi:10.1086/117173

Geach JE, Matsuda Y, Smail I, Chapman SC, Yamada T, Ivison RJ, Hayashino T, Ohta K, Shioya Y, Taniguchi Y (2005) A submillimetre survey of Lyman $\alpha$ haloes in the SA22 protocluster at $z=$ 3.1. Mon Not Roy Astro Soc 363:1398-1408. doi:10.1111/j.1365-2966.2005.09538.x, arXiv:astro$\mathrm{ph} / 0508357$

Gopal-Krishna, Giraud E, Melnick J, della Valle M (1995) A giant Ly $\alpha$ cloud associated with a dust-lane radio galaxy. at $z=2.468$. Astron \& Astrophys 303:705

Gopal-Krishna, Wiita PJ, Barai P (2004) Low-level radio emission from radio galaxies and implications for the large scale structure. J Kor Astron Soc 37:517-525

Graham JA (1998) Shocked gas and star formation in the Centaurus A radio galaxy. Astrophys J 502:245. doi:10.1086/305888

Greve TR, Ivison RJ, Papadopoulos PP (2004) Detection of CO $J=1-0$ in the $z=3.79$ radio galaxy 4C 60.07. Astron \& Astrophys 419:99-107. doi:10.1051/0004-6361:20035788, arXiv:astro-ph/0402464

Greve TR, Stern D, Ivison RJ, De Breuck C, Kovács A, Bertoldi F (2007) Wide-field mid-infrared and millimetre imaging of the high-redshift radio galaxy, 4C41.17. ArXiv e-prints 707, 0707.4482

Griffith MR, Wright AE (1993) The Parkes-MIT-NRAO (PMN) surveys. I-The $4850 \mathrm{MHz}$ surveys and data reduction. Astron J 105:1666-1679. doi:10.1086/116545

Groves BA, Dopita MA, Sutherland RS (2004a) Dusty, radiation pressure-dominated photoionisation. I. Model description, structure, and grids. Astrophys J Suppl 153:9-73. doi:10.1086/421113, arXiv:astro-ph/0404175

Groves BA, Dopita MA, Sutherland RS (2004b) Dusty, radiation pressure-dominated photoionisation. II. Multiwavelength emission line diagnostics for narrow-line regions. Astrophys J Suppl 153:75-91. doi:10.1086/421114, arXiv:astro-ph/0404176

Gurvits LI, Schilizzi RT, Miley GK, Peck A, Bremer MN, Röttgering H, van Breugel W (1997) A compact radio component in $4 \mathrm{C} 41.17$ at $z=3.8$ : a massive clump in a forming galaxy. Astron \& Astrophys 318:11-14

Gurvits LI, Kellermann KI, Frey S (1999) The angular size - redshift relation for compact radio structures in quasars and radio galaxies. Astron \& Astrophys 342:378-388, arXiv:astro-ph/9812018

Haiman Z, Rees MJ (2001) Extended Ly $\alpha$ Emission around young quasars: a constraint on galaxy formation. Astrophys J 556:87-92. doi:10.1086/321567, arXiv:astro-ph/0101174

Haiman Z, Spaans M, Quataert E (2000) Ly $\alpha$ cooling radiation from high-redshift halos. Astrophys J Lett 537:L5-L8. doi:10.1086/312754, arXiv:astro-ph/0003366

Hall PB, Green RF, Cohen M (1998) An optical near-infrared study of radio-loud quasar environments. I. Methods and $Z=1-2$ observations. Astrophys J Suppl 119:1-23. doi:10.1086/313149, arXiv:astro-ph/9806145

Häring N, Rix HW (2004) On the black hole mass-bulge mass relation. Astrophys J Lett 604:L89-L92. doi:10.1086/383567, arXiv:astro-ph/0402376

Hatch NA, Overzier RA, Röttgering HJA, Kurk JD, Miley GK (2007) Diffuse UV light associated with the Spiderweb Galaxy: evidence for in-situ star formation outside galaxies. ArXiv e-prints 710, 0710.4553

Hayashino T, Matsuda Y, Tamura H, Yamauchi R, Yamada T, Ajiki M, Fujita SS, Murayama T, Nagao T, Ohta K, Okamura S, Ouchi M, Shimasaku K, Shioya Y, Taniguchi Y (2004) Large-scale structure of emission-line galaxies at $z=3.1$. Astron J 128:2073-2079. doi:10.1086/424935

Heavens A, Panter B, Jimenez R, Dunlop J (2004) The star-formation history of the Universe from the stellar populations of nearby galaxies. Nature 428:625-627. doi:10.1038/nature02474, arXiv:astro$\mathrm{ph} / 0403293$ 
Heckman TM, Miley GK, Bali ck B, van Breugel WJM, Butcher HR (1982) An optical and radio investigation of the radio galaxy 3C 305. Astrophys J 262:529-553. doi:10.1086/160445

Heckman TM, van Breugel WJM, Miley GK (1984) Emission-line gas associated with the radio lobes of the high-luminosity radio source 3C 171. Astrophys J 286:509-516. doi:10.1086/162626

Hill GJ, Lilly SJ (1991) A change in the cluster environments of radio galaxies with cosmic epoch. Astrophys J 367:1-18. doi:10.1086/169597

Hodapp KW, Kaiser N, Aussel H, Burgett W, Chambers KC, Chun M, Dombeck T, Douglas A, Hafner D, Heasley J, Hoblitt J, Hude C, Isani S, Jedicke R, Jewitt D, Laux U, Luppino GA, Lupton R, Maberry M, Magnier E, Mannery E, Monet D, Morgan J, Onaka P, Price P, Ryan A, Siegmund W, Szapudi I, Tonry J, Wainscoat R, Waterson M (2004) Design of the Pan-STARRS telescopes. Astronomische Nachrichten 325:636-642. doi:10.1002/ansa.200410300

Hopkins PF, Hernquist L, Cox TJ, Di Matteo T, Robertson B, Springel V (2006) A unified, merger-driven model of the origin of starbursts, quasars, the cosmic X-ray background, supermassive black holes, and galaxy spheroids. Astrophys J Suppl 163:1-49. doi:10.1086/499298, arXiv:astro-ph/0506398

Humphrey A, Villar-Martín M, Fosbury R, Vernet J, di Serego Alighieri S (2006) Jet-gas interactions in $z \sim 2.5$ radio galaxies: evolution of the ultraviolet line and continuum emission with radio morphology. Mon Not Roy Astro Soc 369:1103-1114. doi:10.1111/j.1365-2966.2006.10224.x, astro-ph/0602504

Humphrey A, Villar-Martín M, Fosbury R, Binette L, Vernet J, De Breuck C, di Serego Alighieri S (2007) Giant Ly $\alpha$ nebulae around z $>2$ radio galaxies: evidence for infall. Mon Not Roy Astro Soc 375:705714. doi:10.1111/j.1365-2966.2006.11344.x, arXiv:astro-ph/0611778

Intema HT, Venemans BP, Kurk JD, Ouchi M, Kodama T, Röttgering HJA, Miley GK, Overzier RA (2006) Large-scale structure of Lyman break galaxies around a radio galaxy protocluster at $\mathrm{z} \sim 4$. Astron $\&$ Astrophys 456:433-437. doi:10.1051/0004-6361:20064812, arXiv:astro-ph/0606298

Ivison RJ (1995) Detection of dust in the most distant known radio galaxy. Mon Not Roy Astro Soc 275:L33-L36, arXiv:astro-ph/9506039

Ivison RJ, Papadopoulos P, Seaquist ER, Eales SA (1996) A search for molecular gas in a high-redshift radio galaxy. Mon Not Roy Astro Soc 278:669-672

Ivison RJ, Dunlop JS, Smail I, Dey A, Liu MC, Graham JR (2000) An excess of submillimetre sources near 4C 41.17: a candidate protocluster at $Z=3.8$ ? Astrophys J 542:27-34. doi:10.1086/309536, arXiv:astro-ph/0005234

Jaffe W, Bremer MN (1997) Molecular hydrogen emission in cooling flows. Mon Not Roy Astro Soc 284:L1-L5

Jaffe W, Bremer MN, Baker K (2005) HII and $\mathrm{H}_{2}$ in the envelopes of cooling flow central galaxies. Mon Not Roy Astro Soc 360:748-762. doi:10.1111/j.1365-2966.2005.09073.x, arXiv:astro-ph/0504413

Jarvis M, Röttgering HJA (eds) (2003) High-redshift radio galaxies - past, present and future, vol 47

Jarvis MJ, Rawlings S, Eales S, Blundell KM, Bunker AJ, Croft S, McLure RJ, Willott CJ (2001a) A sample of $6 \mathrm{C}$ radio sources designed to find objects at redshift $\mathrm{z}>4$ - III. Imaging and the radio galaxy K-z relation. Mon Not Roy Astro Soc 326:1585-1600. doi:10.1046/j.1365-8711.2001.04730.x, arXiv:astro-ph/0106130

Jarvis MJ, Rawlings S, Lacy M, Blundell KM, Bunker AJ, Eales S, Saunders R, Spinrad H, Stern D, Willott CJ (2001b) A sample of 6C radio sources designed to find objects at redshift $\mathrm{z}>4$ - II. Spectrophotometry and emission-line properties. Mon Not Roy Astro Soc 326:1563-1584. doi:10.1046/j.1365-8711. 2001.04726.x, arXiv:astro-ph/0106127

Jarvis MJ, Rawlings S, Willott CJ, Blundell KM, Eales S, Lacy M (2001c) On the redshift cut-off for steep-spectrum radio sources. Mon Not Roy Astro Soc 327:907-917. doi:10.1046/j.1365-8711.2001. 04778.x, arXiv:astro-ph/0106473

Jarvis MJ, Wilman RJ, Röttgering HJA, Binette L (2003) Probing the absorbing haloes around two highredshift radio galaxies with VLT-UVES*. Mon Not Roy Astro Soc 338:263-272. doi:10.1046/j. 1365-8711.2003.06053.x, arXiv:astro-ph/0209159

Jarvis MJ, Cruz MJ, Cohen AS, Röttgering HJA, Kassim NE (2004) Near-infrared K-band imaging of a sample of ultra-steep-spectrum radio sources selected at 74 MHz. Mon Not Roy Astro Soc 355:20-30. doi:10.1111/j.1365-2966.2004.08278.x, arXiv:astro-ph/0408082

Johnson O, Almaini O, Best PN, Dunlop J (2007) 0.5 Mpc-scale extended X-ray emission in the $z=2.48$ radio galaxy 4C 23.56. Mon Not Roy Astro Soc 376:151-156. doi:10.1111/j.1365-2966.2006.11358. $\mathrm{x}$, arXiv:astro-ph/0608656

Junor W, Biretta JA, Livio M (1999) Formation of the radio jet in M87 at 100 Schwarzschild radii from the central black hole. Nature 401:891-892 
Kajisawa M, Kodama T, Tanaka I, Yamada T, Bower R (2006) Protoclusters with evolved populations around radio galaxies at $\mathrm{z} \sim 2.5$. Mon Not Roy Astro Soc 371:577-582. doi:10.1111/j.1365-2966. 2006.10704.x, arXiv:astro-ph/0606407

Kashikawa N, Kitayama T, Doi M, Misawa T, Komiyama Y, Ota K (2007) The habitat segregation between lyman break galaxies and Ly $\alpha$ emitters around a QSO at z $\sim 5$. Astrophys J 663:765-773. doi:10. 1086/518410, arXiv:0704.2238

Kassim NE, Clarke TE, Cohen AS, Crane PC, Gaussiran T, Gross C, Henning PA, Hicks BC, Junor W, Lane WM, Lazio TJW, Paravastu N, Pihlstrom YM, Polisensky EJ, Ray PS, Stewart KP, Taylor GB, Weiler KW (2006) Exploring the last electromagnetic frontier with the long wavelength array (LWA). In: Long Wavelength Astrophysics, 26th meeting of the IAU, Joint Discussion 12, 21 August 2006, Prague, Czech Republic, JD12, \#56 12

Kauffmann G, White SDM, Guiderdoni B (1993) The Formation and Evolution of Galaxies Within Merging Dark Matter Haloes. Mon Not Roy Astro Soc 264:201

Kaufmann T, Mayer L, Wadsley J, Stadel J, Moore B (2006) Cooling flows within galactic haloes: the kinematics and properties of infalling multiphase gas. Mon Not Roy Astro Soc 370:1612-1622. doi:10. 1111/j.1365-2966.2006.10599.x, arXiv:astro-ph/0507296

Keel WC, Cohen SH, Windhorst RA, Waddington I (1999) Evidence for large-scale structure at $\mathrm{z} \sim 2.4$ from Ly $\alpha$ imaging. Astron J 118:2547-2560. doi:10.1086/301139, arXiv:astro-ph/9908183

Kellermann KI (2003) Variability, brightness temperature, superluminal motion, doppler boosting, and related issues. In: Zensus JA, Cohen $\mathrm{MH}$, Ros E (eds) Radio astronomy at the fringe, Astronomical Society of the Pacific conference series, vol 300, p 185

Klamer IJ, Ekers RD, Sadler EM, Hunstead RW (2004) Molecular gas at high redshift: jet-induced star formation? Astrophys J Lett 612:L97-L100. doi:10.1086/424843, arXiv:astro-ph/0408015

Klamer IJ, Ekers RD, Sadler EM, Weiss A, Hunstead RW, De Breuck C (2005) CO (1-0) and CO (5-4) observations of the most distant known radio galaxy at $z=5.2$. Astrophys J Lett 621:L1-L4. doi:10. 1086/429147, arXiv:astro-ph/0501447

Klamer IJ, Ekers RD, Bryant JJ, Hunstead RW, Sadler EM, De Breuck C (2006) A search for distant radio galaxies from SUMSS and NVSS - III. Radio spectral energy distributions and the z- $\alpha$ correlation. Mon Not Roy Astro Soc 371:852-866. doi:10.1111/j.1365-2966.2006.10714.x, astro-ph/0606469

Knopp GP, Chambers KC (1997) Dust at high redshift: infrared and optical polarimetry of an ultra-steepspectrum radio source at $Z=2.572$. Astrophys $\mathrm{J} 487: 644$. doi:10.1086/304656

Kodama T, Bower R (2003) The $K_{S}$-band luminosity and stellar mass functions of galaxies in $z=1$ clusters. Mon Not Roy Astro Soc 346:1-12. doi:10.1046/j.1365-2966.2003.07093.x, arXiv:astro-ph/0308130

Kodama T, Tanaka I, Kajisawa M, Kurk J, Venemans B, De Breuck C, Vernet J, Lidman C (2007) The first appearance of the red sequence of galaxies in proto-clusters at $2<z<3$. Mon Not Roy Astro Soc 377:1717-1725. doi:10.1111/j.1365-2966.2007.11739.x, arXiv:astro-ph/0703382

Kopylov AI, Goss WM, Parï̌skii YN, Soboleva NS, Verkhodanov OV, Temirova AV, Zhelenkova OP (2006) RC J0311 + 0507: A candidate for superpowerful radio galaxies in the early universe at redshift $z=4.514$. Astro Letters 32:433-438. doi:10.1134/S1063773706070012, arXiv:0705.2971

Kormendy J, Gebhardt K (2001) Supermassive Black Holes in Galactic Nuclei (Plenary Talk). In: Wheeler JC, Martel H (eds) 20th Texas Symposium on relativistic astrophysics, American Institute of physics conference series, vol 586, p 363

Krause M (2005) Galactic wind shells and high redshift radio galaxies. On the nature of associated absorbers. Astron \& Astrophys 436:845-851. doi:10.1051/0004-6361:20042450, arXiv:astro-ph/0503322

Kurk JD, Röttgering HJA, Pentericci L, Miley GK, van Breugel W, Carilli CL, Ford H, Heckman T, McCarthy P, Moorwood A (2000) A Search for clusters at high redshift. I. Candidate Ly alpha emitters near 1138-262 at $z=2.2$. Astron \& Astrophys 358:L1-L4, astro-ph/0005058

Kurk JD, Pentericci L, Overzier RA, Röttgering HJA, Miley GK (2004a) A search for clusters at high redshift. IV. Spectroscopy of $\mathrm{H} \alpha$ emitters in a proto-cluster at $z=2.16$. Astron \& Astrophys 428:817-821. doi:10.1051/0004-6361:20041819, astro-ph/0410203

Kurk JD, Pentericci L, Röttgering HJA, Miley GK (2004b) A search for clusters at high redshift. III. Candidate $\mathrm{H} \alpha$ emitters and EROs in the PKS 1138-262 proto-cluster at $z=2.16$. Astron \& Astrophys 428:793-815. doi:10.1051/0004-6361:20040075, astro-ph/0410202

Lacy M, Miley G, Rawlings S, Saunders R, Dickinson M, Garrington S, Maddox S, Pooley G, Steidel C, Bremer MN, Cotter G, Ojik Rvan, Rottgering H, Warner P (1994) 8C 1435 + 635: a radio galaxy at $z=4.25$. Mon Not Roy Astro Soc 271:504-512 
Lacy M, Rawlings S, Hill GJ, Bunker AJ, Ridgway SE, Stern D (1999) Optical spectroscopy of two overlapping, flux-density-limited samples of radio sources in the North Ecliptic Cap, selected at 38 and 151MHz. Mon Not Roy Astro Soc 308:1096-1116, arXiv:astro-ph/9905358

Large MI, Mills BY, Little AG, Crawford DF, Sutton JM (1981) The Molonglo Reference Catalogue of Radio Sources. Mon Not Roy Astro Soc 194:693

Larson R (1992) Galaxy formation and evolution. In: Tenorio-Tagle G, Prieto M, Sanchez F (eds) Star formation in stellar systems, p 125

Le Fevre O, Deltorn JM, Crampton D, Dickinson M (1996) Clustering around the radio galaxy MRC 0316-257 at $Z=3.14$. Astrophys J Lett 471:L11. doi:10.1086/310319, arXiv:astro-ph/9609020

Leckrone DS, Cheng ES, Feinberg LD, Trauger JT, Macchetto FD, MacKenty JW (1998) Wide Field Camera 3 (WFC3) - A Facility Instrument For The Hubble Space Telescope. In: Bulletin of the American Astronomical Society, Bulletin of the American Astronomical Society, vol 30, p 861

Li Y, Hernquist L, Robertson B, Cox TJ, Hopkins PF, Springel V, Gao L, Di Matteo T, Zentner AR, Jenkins A, Yoshida N (2007) Formation of $\mathrm{z} \sim 6$ quasars from hierarchical galaxy mergers. Astrophys J 665:187-208. doi:10.1086/519297, arXiv:astro-ph/0608190

Lilly SJ (1988) Discovery of a radio galaxy at a redshift of 3.395. Astrophys J 333:161-167. doi:10.1086/ 166732

Lilly SJ (1989) Faint identifications of 1 Jansky radio source empty fields - Radio galaxies at high redshift. Astrophys J 340:77-89. doi:10.1086/167377

Lilly SJ, Longair MS (1984) Stellar populations in distant radio galaxies. Mon Not Roy Astro Soc 211:833855

Lilly SJ, Le Fevre O, Hammer F, Crampton D (1996) The Canada-France redshift survey: the luminosity density and star formation history of the universe to $\mathrm{Z}$ approximately 1. Astrophys J Lett 460:L1. doi:10.1086/309975, arXiv:astro-ph/9601050

Lynden-Bell D (1969) Galactic nuclei as collapsed old quasars. Nature 223:690

Madau P, Pozzetti L, Dickinson M (1998) The star formation history of field galaxies. Astrophys J 498:106. doi:10.1086/305523, arXiv:astro-ph/9708220

Magorrian J, Tremaine S, Richstone D, Bender R, Bower G, Dressler A, Faber SM, Gebhardt K, Green R, Grillmair C, Kormendy J, Lauer T (1998) The demography of massive dark objects in galaxy centers. Astron J 115:2285-2305. doi:10.1086/300353, arXiv:astro-ph/9708072

Manzini A, di Serego Alighieri S (1996) Dust scattering in radio galaxies. Astron Astrophys 311:79-94

Matsuda Y, Yamada T, Hayashino T, Tamura H, Yamauchi R, Ajiki M, Fujita SS, Murayama T, Nagao T, Ohta K, Okamura S, Ouchi M, Shimasaku K, Shioya Y, Taniguchi Y (2004) A Subaru search for Ly $\alpha$ blobs in and around the protocluster region at redshift $z=3.1$. Astron J 128:569-584. doi:10.1086/ 422020, arXiv:astro-ph/0405221

Matsuda Y, Yamada T, Hayashino T, Tamura H, Yamauchi R, Murayama T, Nagao T, Ohta K, Okamura S, Ouchi M, Shimasaku K, Shioya Y, Taniguchi Y (2005) Large-scale filamentary structure around the protocluster at redshift $z=3.1$. Astrophys J Lett 634:L125-L128. doi:10.1086/499071, arXiv:astro$\mathrm{ph} / 0510762$

Mauch T, Murphy T, Buttery HJ, Curran J, Hunstead RW, Piestrzynski B, Robertson JG, Sadler EM (2003) SUMSS: a wide-field radio imaging survey of the southern sky-II. The source catalogue. Mon Not Roy Astro Soc 342:1117-1130. doi:10.1046/j.1365-8711.2003.06605.x, arXiv:astro-ph/0303188

McCarthy PJ (1991) High redshift radio galaxies from the Third Bologna Catalogue. Astron J 102:518-521. doi:10.1086/115890

McCarthy PJ (1993) High redshift radio galaxies. ARA\& A 31:639-688. doi:10.1146/annurev.aa.31. 090193.003231

McCarthy PJ, van Breugel W, Spinrad H, Djorgovski S (1987) A correlation between the radio and optical morphologies of distant 3Cr radio galaxies. Astrophys J Lett 321:L29-L33. doi:10.1086/185000

McCarthy PJ, Kapahi VK, van Breugel W, Subrahmanya CR (1990) High-redshift radio galaxies from the Molonglo Catalogue. Astron J 100:1014-1027. doi:10.1086/115575

McCarthy PJ, van Breugel W, Kapahi VK, Subrahmanya CR (1991) High redshift radio galaxies from the Molonglo Catalogue. II. Astron J 102:522-536. doi:10.1086/115891

McCarthy PJ, Kapahi VK, van Breugel W, Persson SE, Athreya R, Subrahmanya CR (1996) The Molonglo Reference Catalog/1 Jansky Radio Source Survey. I. Radio galaxy identifications. Astrophys J Suppl 107:19. doi:10.1086/192353

McLure RJ, Jarvis MJ (2002) Measuring the black hole masses of high-redshift quasars. Mon Not Roy Astro Soc 337:109-116. doi:10.1046/j.1365-8711.2002.05871.x, arXiv:astro-ph/0204473 
McNamara BR, Wise M, Nulsen PEJ, David LP, Sarazin CL, Bautz M, Markevitch M, Vikhlinin A, Forman WR, Jones C, Harris DE (2000) Chandra X-ray observations of the hydra A cluster: an interaction between the radio source and the X-ray-emitting gas. Astrophys J Lett 534:L135-L138. doi:10.1086/ 312662, arXiv:astro-ph/0001402

Menci N (2004) Co-evolution of Galaxies and AGNs in Hierarchical Galaxy Formation Models. APSS 294:15-22. doi:10.1007/s10509-004-4013-7

Miley G (1980) The structure of extended extragalactic radio sources. ARA\& A 18:165-218. doi:10.1146/ annurev.aa.18.090180.001121

Miley GK (1968) Variation of the angular sizes of quasars with red-shift. Nature 218:933

Miley GK, Overzier RA, Tsvetanov ZI, Bouwens RJ, Benítez N, Blakeslee JP, Ford HC, Illingworth GD, Postman M, Rosati P, Clampin M, Hartig GF, Zirm AW, Röttgering HJA, Venemans BP, Ardila DR, Bartko F, Broadhurst TJ, Brown RA, Burrows CJ, Cheng ES, Cross NJG, De Breuck C, Feldman PD, Franx M, Golimowski DA, Gronwall C, Infante L, Martel AR, Menanteau F, Meurer GR, Sirianni M, Kimble RA, Krist JE, Sparks WB, Tran HD, White RL, Zheng W (2004) A large population of Lyman-break galaxies in a protocluster at redshift $\mathrm{z} \sim 4$.1. Nature 427:47-50, astro-ph/0401034

Miley GK, Overzier RA, Zirm AW, Ford HC, Kurk J, Pentericci L, Blakeslee JP, Franx M, Illingworth GD, Postman M, Rosati P, Röttgering HJA, Venemans BP, Helder E (2006) The Spiderweb Galaxy: a forming massive cluster galaxy at $\mathrm{z} \sim 2$. Astrophys J Lett 650:L29-L32. doi:10.1086/508534, arXiv:astro-ph/0610909

Minkowski R (1960) A New Distant Cluster of Galaxies. Astrophys J 132:908-910. doi:10.1086/146994

Miyoshi M, Moran J, Herrnstein J, Greenhill L, Nakai N, Diamond P, Inoue M (1995) Evidence for a Black-Hole from High Rotation Velocities in a Sub-Parsec Region of NGC4258. Nature 373:127. doi: $10.1038 / 373127 \mathrm{a} 0$

Möller P, Fynbo JU (2001) Detection of a redshift 3.04 filament. Astron \& Astrophys 372:L57-L60. doi:10. 1051/0004-6361:20010606, arXiv:astro-ph/0105114

Monaco P, Møller P, Fynbo JPU, Weidinger M, Ledoux C, Theuns T (2005) Tracing large-scale structure at high redshift with Lyman- $\alpha$ emitters: the effect of peculiar velocities. Astron \& Astrophys 440:799-808. doi:10.1051/0004-6361:20042570, arXiv:astro-ph/0505477

Morganti R (2006) Neutral hydrogen in radio galaxies: Results from nearby, importance for far away. Astron Nachrich 327:127-134. doi:10.1002/ansa.200510492, arXiv:astro-ph/0510261

Nakata F, Kajisawa M, Yamada T, Kodama T, Shimasaku K, Tanaka I, Doi M, Furusawa H, Hamabe M, Iye M, Kimura M, Komiyama Y, Miyazaki S, Okamura S, Ouchi M, Sasaki T, Sekigushi M, Yagi M, Yasuda N (2002) Galaxy Population in the Cluster of Galaxies around the Radio Galaxy 3C324 at $z=1.2$. In: Borgani S, Mezzetti M, Valdarnini R (eds) Tracing Cosmic Evolution with Galaxy Clusters, Astronomical Society of the Pacific Conference Series, vol 268, pp 421

Napier PJ (2006) The EVLA Project: Ten Times More Capability for the VLA. In: Backer DC, Moran JM, Turner JL (eds) Revealing the Molecular Universe: One Antenna is Never Enough, Astronomical Society of the Pacific Conference Series, vol 356, pp 65

Neeser MJ, Eales SA, Law-Green JD, Leahy JP, Rawlings S (1995) The Linear-Size Evolution of Classical Double Radio Sources. Astrophys J 451:76. doi:10.1086/176201, arXiv:astro-ph/9508036

Nesvadba NPH, Lehnert MD, Eisenhauer F, Gilbert A, Tecza M, Abuter R (2006) Extreme Gas Kinematics in the $z=2.2$ Powerful Radio Galaxy MRC 1138-262: Evidence for Efficient Active Galactic Nucleus Feedback in the Early Universe? Astrophys J 650:693-705. doi:10.1086/507266, arXiv:astro$\mathrm{ph} / 0606530$

Nilsson K, Valtonen MJ, Kotilainen J, Jaakkola T (1993) On the redshift-apparent size diagram of double radio sources. Astrophys J 413:453-476. doi:10.1086/173016

Nilsson KK, Fynbo JPU, Møller P, Sommer-Larsen J, Ledoux C (2006) A Lyman- $\alpha$ blob in the GOODS South field: evidence for cold accretion onto a dark matter halo. Astron \& Astrophys 452:L23-L26. doi:10.1051/0004-6361:200600025, arXiv:astro-ph/0512396

Novak GS, Faber SM, Dekel A (2006) On the Correlations of Massive Black Holes with Their Host Galaxies. Astrophys J 637:96-103. doi:10.1086/498333, arXiv:astro-ph/0510102

Omont A (2007) Molecules in galaxies. Reports of Progress in Physics 70:1099-1176. doi:10.1088/ 0034-4885/70/7/R03

Osterbrock DE, Ferland GJ (2006) Astrophysics of gaseous nebulae and active galactic nuclei. Astrophysics of gaseous nebulae and active galactic nuclei, 2nd ed by D.E. Osterbrock and G.J. Ferland. Sausalito, CA: University Science Books, 2006 
Ouchi M, Shimasaku K, Akiyama M, Sekiguchi K, Furusawa H, Okamura S, Kashikawa N, Iye M, Kodama T, Saito T, Sasaki T, Simpson C, Takata T, Yamada T, Yamanoi H, Yoshida M, Yoshida M (2005) The Discovery of Primeval Large-Scale Structures with Forming Clusters at Redshift 6. Astrophys J Lett 620:L1-L4. doi:10.1086/428499, arXiv:astro-ph/0412648

Overzier RA (2006) Emergence of Cosmic Structures around Distant Radio Galaxies and Quasars. PhD thesis, Leiden Observatory, Leiden University, P.O. Box 9513, 2300 RA Leiden, The Netherlands

Overzier RA, Harris DE, Carilli CL, Pentericci L, Röttgering HJA, Miley GK (2005) On the X-ray emission of $\mathrm{z} \sim 2$ radio galaxies: IC scattering of the $\mathrm{CMB}$ and no evidence for fully-formed potential wells. Astron \& Astrophys 433:87-100. doi:10.1051/0004-6361:2004165, arXiv:astro-ph/0412026

Overzier RA, Bouwens RJ, Cross NJG, Venemans B, Miley GK, Zirm AW, Benitez N, Blakeslee JP, Coe D, Demarco R, Ford H, Homeier N, Illingworth GD, Kurk JD, Martel A, Mei S, Rottgering HJA, Tsvetanov Z, Zheng W (2006a) Star formation, morphologies and clustering of galaxies in a radio galaxy protocluster at $z=4.1$. ArXiv Astrophysics e-prints astro-ph/0601223

Overzier RA, Miley GK, Bouwens RJ, Cross NJG, Zirm AW, Benítez N, Blakeslee JP, Clampin M, Demarco R, Ford HC, Hartig GF, Illingworth GD, Martel AR, Röttgering HJA, Venemans B, Ardila DR, Bartko F, Bradley LD, Broadhurst TJ, Coe D, Feldman PD, Franx M, Golimowski DA, Goto T, Gronwall C, Holden B, Homeier N, Infante L, Kimble RA, Krist JE, Mei S, Menanteau F, Meurer GR, Motta V, Postman M, Rosati P, Siriann M, Sparks WB, Tran HD, Tsvetanov ZI, White RL, Zheng W (2006b) Clustering of Star-forming Galaxies Near a Radio Galaxy at $z=5.2$. Astrophys J 637:58-73. doi:10. 1086/498234, arXiv:astro-ph/0509308

Owen FN, Keel WC (1995) Faint B3 radio galaxies: Luminosity versus evolution effects. Astron J 109:486499. doi:10.1086/117294

Pahre MA, Djorgovski SG (1995) A Near-Infrared Search for Line Emission from Protogalaxies Using the W. M. Keck Telescope. Astrophys J Lett 449:L1. doi:10.1086/309632, arXiv:astro-ph/9506043

Panter B, Jimenez R, Heavens AF, Charlot S (2007) The star formation histories of galaxies in the Sloan Digital Sky Survey. Mon Not Roy Astro Soc 378:1550-1564. doi:10.1111/j.1365-2966.2007.11909. $\mathrm{x}$, arXiv:astro-ph/0608531

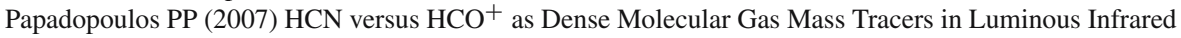
Galaxies. Astrophys J 656:792-797. doi:10.1086/510186, arXiv:astro-ph/0610477

Papadopoulos PP, Röttgering HJA, van der Werf PP, Guilloteau S, Omont A, van Breugel WJM, Tilanus RPJ (2000) CO (4-3) and Dust Emission in Two Powerful High-Z Radio Galaxies, and CO Lines at High Redshifts. Astrophys J 528:626-636. doi:10.1086/308215, arXiv:astro-ph/9908286

Papadopoulos PP, Greve TR, Ivison RJ, De Breuck C (2005) A sensitive search for CO $J=1-0$ emission in 4C 41.17: high-excitation molecular gas at $z=3.8$. Astron \& Astrophys 444:813-819. doi:10. 1051/0004-6361:20053611, arXiv:astro-ph/0509048

Pascarelle SM, Windhorst RA, Keel WC, Odewahn SC (1996) Sub-galactic clumps at a redshift of 2.39 and implications for galaxy formation. Nature 383:45-50. doi:10.1038/383045a0

Peacock JA (1999) Cosmological Physics. Cosmological Physics, by John A. Peacock, pp. 704. ISBN 052141072X. Cambridge, UK: Cambridge University Press, January 1999

Pei YC (1995) The luminosity function of quasars. Astrophys J 438:623-631. doi:10.1086/175105

Pentericci L, Röttgering HJA, Miley GK, Carilli CL, McCarthy P (1997) The radio galaxy 1138-262 at $z=2.2$ : a giant elliptical galaxy at the center of a proto-cluster? Astron \& Astrophys 326:580-596

Pentericci L, Röttgering HJA, Miley GK, Spinrad H, McCarthy PJ, van Breugel WJM, Macchetto F (1998) HST Images of the Extremely Clumpy Radio Galaxy 1138-262 at $Z=2.2$. Astrophys J 504:139. doi:10.1086/306087

Pentericci L, Röttgering HJA, Miley GK, McCarthy P, Spinrad H, van Breugel WJM, Macchetto F (1999) HST images and properties of the most distant radio galaxies. Astron \& Astrophys 341:329-347, astro-ph/9809056

Pentericci L, Kurk JD, Röttgering HJA, Miley GK, van Breugel W, Carilli CL, Ford H, Heckman T, McCarthy P, Moorwood A (2000a) A search for clusters at high redshift. II. A proto cluster around a radio galaxy at $z=2.16$. Astron \& Astrophys 361:L25-L28, astro-ph/0008143

Pentericci L, Van Reeven W, Carilli CL, Röttgering HJA, Miley GK (2000b) VLA radio continuum observations of a new sample of high redshift radio galaxies. Astron \& Astrophys Suppl 145:121-159, astro-ph/0005524

Pentericci L, McCarthy PJ, Röttgering HJA, Miley GK, van Breugel WJM, Fosbury R (2001) NICMOS Observations of High-Redshift Radio Galaxies: Witnessing the Formation of Bright Elliptical Galaxies? Astrophys J Suppl 135:63-85. doi:10.1086/321781, arXiv:astro-ph/0102323 
Pentericci L, Kurk JD, Carilli CL, Harris DE, Miley GK, Röttgering HJA (2002) A Chandra study of X-ray sources in the field of the $z=2.16$ radio galaxy MRC 1138-262. Astron \& Astrophys 396:109-115. doi:10.1051/0004-6361:20021368, arXiv:astro-ph/0209392

Pérez-Torres MA, De Breuck C (2005) The extremely asymmetric radio structure of the $z=3.1$ radio galaxy B3 J2330 + 3927. Mon Not Roy Astro Soc 363:L41-L45. doi:10.1111/j.1745-3933.2005.00080. $\mathrm{x}$, arXiv:astro-ph/0507489

Pérez-Torres MA, De Breuck C, van Breugel W, Miley G (2006) Very high-resolution radio observations of HzRGs. Astron Nachrich 327:245-248. doi:10.1002/ansa.200510516, arXiv:astro-ph/0508692

Peterson BM (1997) An introduction to active galactic nuclei. Cambridge University Press, Cambridge, Physical description xvi, 238 pp. ISBN 0521473489

Podariu S, Daly RA, Mory MP, Ratra B (2003) Radio galaxy redshift-angular size data constraints on dark energy. Astrophys J 584:577-579. doi:10.1086/345752, arXiv:astro-ph/0207096

Rawlings S, Saunders R (1991) Evidence for a common central-engine mechanism in all extragalactic radio sources. Nature 349:138-140. doi:10.1038/349138a0

Rawlings S, Eales S, Warren S (1990) The detection of four high-redshift ( 0.5 much less than Z less than or equal to 3.22) radiogalaxies by optical spectroscopy of five blank fields. Mon Not Roy Astro Soc 243:14P-18P

Rawlings S, Lacy M, Blundell KM, Eales SA, Bunker AJ, Garrington ST (1996) A radio galaxy at redshift 4.41. Nature 383:502-505. doi:10.1038/383502a0

Rees MJ (1967) Studies in radio source structure-I. A relativistically expanding model for variable quasistellar radio sources. Mon Not Roy Astro Soc 135:345

Rees MJ (1989) The radio/optical alignment of high-z radio galaxies - Triggering of star formation in radio lobes. Mon Not Roy Astro Soc 239:1P-4P

Rengelink RB (1999) The Westerbork Northern Sky Survey; the cosmological evolution of radio sources. $\mathrm{PhD}$ thesis, Leiden Observatory, Leiden University, Leiden, The Netherlands

Rengelink RB, Tang Y, Bruyn AGde, Miley GK, Bremer MN, Roettgering HJA, Bremer MAR (1997) The Westerbork Northern Sky Survey (WENSS), I. A 570 square degree Mini-Survey around the North Ecliptic Pole. Astron \& Astrophys Suppl 124:259-280

Reuland M (2005) Gas, dust and star formation in distant radio galaxies. PhD thesis, Leiden Observatory, Leiden University, Leiden, The Netherlands

Reuland M, van Breugel W, Röttgering H, de Vries W, De Breuck C, Stern D (2003a) An obscured radio galaxy at high redshift. Astrophys J Lett 582:L71-L74. doi:10.1086/367689, arXiv:astro-ph/0302432

Reuland M, van Breugel W, Röttgering H, de Vries W, Stanford SA, Dey A, Lacy M, Bland-Hawthorn J, Dopita M, Miley G (2003b) Giant Ly $\alpha$ nebulae associated with high-redshift radio galaxies. Astrophys J 592:755-766. doi:10.1086/375619, arXiv:astro-ph/0303637

Reuland M, Röttgering H, van Breugel W, De Breuck C (2004) Dust and star formation in distant radio galaxies. Mon Not Roy Astro Soc 353:377-390. doi:10.1111/j.1365-2966.2004.08063.x, arXiv:astro$\mathrm{ph} / 0405567$

Reuland M, van Breugel W, de Vries W, Dopita MA, Dey A, Miley G, Röttgering H, Venemans B, Stanford SA, Lacy M, Spinrad H, Dawson S, Stern D, Bunker A (2007) Metal-enriched gaseous halos around distant radio galaxies: clues to Feedback in galaxy formation. Astron J 133:2607-2623. doi:10.1086/ 516571, arXiv:astro-ph/0702753

Rocca-Volmerange B, Remazeilles M (2005) Evidence of intense hot ( $340 \mathrm{~K})$ dust emission in 3CR radio galaxies. The most dissipative source of cooling in AGNs. Astron \& Astrophys 433:73-77. doi:10. 1051/0004-6361:20042106, arXiv:astro-ph/0411733

Rocca-Volmerange B, Le Borgne D, De Breuck C, Fioc M, Moy E (2004) The radio galaxy K-z relation: the $10^{12} \mathrm{M}_{\text {Sol }}$ mass limit. Masses of galaxies from the $\mathrm{L}_{K}$ luminosity, up to $\mathrm{z}>4$. Astron \& Astrophys 415:931-940. doi:10.1051/0004-6361:20031717, arXiv:astro-ph/0311490

Röttgering H, de Bruyn G, Pentericci L (1999a) HI and high redshift radio galaxies. In: Röttgering HJA, Best PN, Lehnert MD (eds) The most distant radio galaxies, pp 113

Röttgering HJA, Lacy M, Miley GK, Chambers KC, Saunders R (1994) Samples of ultra-steep spectrum radio sources. Astron \& Astrophys Suppl 108:79-141

Röttgering HJA, Miley GK, Chambers KC, Macchetto F (1995) CCD imaging of ultra-steep-spectrum radio sources. Astron \& Astrophys Suppl 114:51

Röttgering HJA, West MJ, Miley GK, Chambers KC (1996) The optical counterparts and the environments of ultra-steep-spectrum radio sources. Astron \& Astrophys 307:376-384

Röttgering HJA, van Ojik R, Miley GK, Chambers KC, van Breugel WJM, de Koff S (1997) Spectroscopy of ultra-steep spectrum radio sources: a sample of $\mathrm{z}>2$ radio galaxies. Astron \& Astrophys 326:505-527, arXiv:astro-ph/9608063 
Röttgering HJA, Best PN, Lehnert MD (eds) (1999b) The most distant radio galaxies

Röttgering HJA, Best PN, De Breuck C, Kurk J, Pentericci L, van Breugel W (2000) Distant radio galaxies: the present and future. In: van Haarlem MP (ed) Perspectives on Radio Astro: science with large antenna arrays, proceedings of the conference on at The Royal Netherlands Academy of Arts and Sciences, Amsterdam, 7-9 April 1999. ASTRON,, 340 pp. ISBN: 90-805434-1-1

Röttgering HJA, Braun R, Barthel PD, van Haarlem MP, Miley GK, Morganti R, Snellen I, Falcke H, de Bruyn AG, Stappers RB, Boland WHWM, Butcher HR, de Geus EJ, Koopmans L, Fender R, Kuijpers J, Schilizzi RT, Vogt C, Wijers RAMJ, Wise M, Brouw WN, Hamaker JP, Noordam JE, Oosterloo T, Bahren L, Brentjens MA, Wijnholds SJ, Bregman JD, van Cappellen WA, Gunst AW, Kant GW, Reitsma J, van der Schaaf K, de Vos CM (2006) LOFAR_Opening up a new window on the Universe. ArXiv Astrophysics e-prints astro-ph/0610596

Scharf C, Smail I, Ivison R, Bower R, van Breugel W, Reuland M (2003) Extended X-Ray Emission around 4C 41.17 at $z=3.8$. Astrophys J 596:105-113. doi:10.1086/377531, arXiv:astro-ph/0306314

Schilizzi RT, van Ardenne A, Miley GK, Baud B, Baath L, Ronnang BO, Pauliny-Toth IIK (1979) High resolution observations of the compact central component in the giant radio source $3 \mathrm{C} 236$. Astron \& Astrophys 77:1-2

Schiminovich D, Ilbert O, Arnouts S, Milliard B, Tresse L, Le Fèvre O, Treyer M, Wyder TK, Budavári T, Zucca E, Zamorani G, Martin DC, Adami C, Arnaboldi M, Bardelli S, Barlow T, Bianchi L, Bolzonella M, Bottini D, Byun YI, Cappi A, Contini T, Charlot S, Donas J, Forster K, Foucaud S, Franzetti P, Friedman PG, Garilli B, Gavignaud I, Guzzo L, Heckman TM, Hoopes C, Iovino A, Jelinsky P, Le Brun V, Lee YW, Maccagni D, Madore BF, Malina R, Marano B, Marinoni C, McCracken HJ, Mazure A, Meneux B, Morrissey P, Neff S, Paltani S, Pellò R, Picat JP, Pollo A, Pozzetti L, Radovich M, Rich RM, Scaramella R, Scodeggio M, Seibert M, Siegmund O, Small T, Szalay AS, Vettolani G, Welsh B, $\mathrm{Xu}$ CK, Zanichelli A (2005) The GALEX-VVDS measurement of the evolution of the far-ultraviolet luminosity density and the cosmic star formation rate. Astrophys J Lett 619:L47-L50. doi:10.1086/ 427077, arXiv:astro-ph/0411424

Schwartz DA (2002) X-ray jets as cosmic beacons. Astrophys J Lett 569:L23-L26. doi:10.1086/340482

Scoville NZ, Yun MS, Windhorst RA, Keel WC, Armus L (1997) CO J = 3-2 emission in the radio galaxy 53W002 at it $Z=2.394$. Astrophys J Lett 485:L21. doi:10.1086/310807, arXiv:astro-ph/9706291

Seymour N, Stern D, De Breuck C, Vernet J, Rettura A, Dickinson M, Dey A, Eisenhardt P, Fosbury R, Lacy M, McCarthy P, Miley G, Rocca-Volmerange B, Röttgering H, Stanford SA, Teplitz H, van Breugel W, Zirm A (2007) The massive hosts of radio galaxies across cosmic time. Astrophys J Suppl 171:353-375. doi:10.1086/517887, arXiv:astro-ph/0703224

Shimasaku K, Ouchi M, Okamura S, Kashikawa N, Doi M, Furusawa H, Hamabe M, Hayashino T, Kawabata K, Kimura M, Kodaira K, Komiyama Y, Matsuda Y, Miyazaki M, Miyazaki S, Nakata F, Ohta K, Ohyama Y, Sekiguchi M, Shioya Y, Tamura H, Taniguchi Y, Yagi M, Yamada T, Yasuda N (2003) Subaru Deep Survey. IV. Discovery of a large-scale structure at redshift $\sim=5$. Astrophys J Lett 586:L111-L114. doi:10.1086/374880, arXiv:astro-ph/0302466

Siebenmorgen R, Krügel E (2007) Dust in starburst nuclei and ULIRGs. SED models for observers. Astron \& Astrophys 461:445-453. doi:10.1051/0004-6361:20065700, arXiv:astro-ph/0606444

Siebenmorgen R, Krügel E, Laureijs RJ (2001) The infrared continuum radiation of NGC 1808. A PAH and polarisation study. Astron \& Astrophys 377:735-744. doi:10.1051/0004-6361:20010969, arXiv:astro-ph/0107098

Silk J, Rees MJ (1998) Quasars and galaxy formation. Astron \& Astrophys 331:L1-L4, arXiv:astro$\mathrm{ph} / 9801013$

Simpson C, Eisenhardt P (1999) The detection and photometric redshift determination of distant galaxies using SIRTF's infrared array camera. PASP 111:691-701, arXiv:astro-ph/9903067

Smail I, Ivison RJ, Gilbank DG, Dunlop JS, Keel WC, Motohara K, Stevens JA (2003) A SCUBA galaxy in the protocluster around 53W002 at $z=2.4$. Astrophys J 583:551-558. doi:10.1086/345474, arXiv:astro-ph/0210183

Smith HE, Spinrad H (1980) An update of the status of the revised 3C catalog of radio sources 22 new galaxy redshifts. PASP 92:553-569

Solomon PM, Vanden Bout PA (2005) Molecular gas at high redshift. ARA\&A 43:677-725, arXiv:astro$\mathrm{ph} / 0508481$

Solórzano-Iñarrea C, Best PN, Röttgering HJA, Cimatti A (2004) VLT spectropolarimetry of two powerful radio galaxies at $\mathrm{z} \sim 1.4$ : ultraviolet continuum, emission-line properties and the nature of high-redshift dust. Mon Not Roy Astro Soc 351:997-1014. doi:10.1111/j.1365-2966.2004.07842.x, arXiv:astro-ph/0403390 
Spinrad H (1976) Following Minkowski's footsteps - A survey of distant radio sources. PASP 88:565-573

Spinrad H, Westphal J, Kristian J, Sandage A (1977) Spectroscopy and photometry of the distant radio galaxy 3C 343.1. Astrophys J Lett 216:L87-L89

Springel V, Di Matteo T, Hernquist L (2005a) Modelling feedback from stars and black holes in galaxy mergers. Mon Not Roy Astro Soc 361:776-794. doi:10.1111/j.1365-2966.2005.09238.x, arXiv:astro$\mathrm{ph} / 0411108$

Springel V, White SDM, Jenkins A, Frenk CS, Yoshida N, Gao L, Navarro J, Thacker R, Croton D, Helly J, Peacock JA, Cole S, Thomas P, Couchman H, Evrard A, Colberg J, Pearce F (2005b) Simulations of the formation, evolution and clustering of galaxies and quasars. Nature 435:629-636. doi:10.1038/ nature03597, arXiv:astro-ph/0504097

Stanford SA, Eisenhardt PR, Brodwin M, Gonzalez AH, Stern D, Jannuzi BT, Dey A, Brown MJI, McKenzie E, Elston R (2005) An IR-selected galaxy cluster at $z=1.41$. Astrophys J Lett 634:L129_ L132. doi:10.1086/499045, arXiv:astro-ph/0510655

Steidel CC, Adelberger KL, Dickinson M, Giavalisco M, Pettini M, Kellogg M (1998) A large structure of galaxies at redshift Z approximately 3 and its cosmological implications. Astrophys J 492:428. doi:10. 1086/305073, arXiv:astro-ph/9708125

Steidel CC, Adelberger KL, Shapley AE, Pettini M, Dickinson M, Giavalisco M (2000) Ly $\alpha$ Imaging of a Proto-Cluster Region at $z=3.09$. Astrophys J 532:170-182. doi:10.1086/308568, arXiv:astro$\mathrm{ph} / 9910144$

Steidel CC, Adelberger KL, Shapley AE, Erb DK, Reddy NA, Pettini M (2005) Spectroscopic Identification of a Protocluster at $z=2.300$ : environmental dependence of galaxy properties at high redshift. Astrophys J 626:44-50. doi:10.1086/429989, arXiv:astro-ph/0502432

Stern D, Dey A, Spinrad H, Maxfield L, Dickinson M, Schlegel D, González RA (1999) New high-redshift radio galaxies from the MIT-Green Bank Catalog. Astron J 117:1122-1138. doi:10.1086/300770, arXiv:astro-ph/9811344

Stevens JA, Ivison RJ, Dunlop JS, Smail IR, Percival WJ, Hughes DH, Röttgering HJA, van Breugel WJM, Reuland M (2003) The formation of cluster elliptical galaxies as revealed by extensive star formation. Nature 425:264-267, arXiv:astro-ph/0309495

Straughn AN, Cohen SH, Ryan RE, Hathi NP, Windhorst RA, Jansen RA (2006) Tracing galaxy assembly: tadpole galaxies in the hubble ultra deep field. Astrophys J 639:724-730. doi:10.1086/499576, arXiv:astro-ph/0511423

Suwa T, Habe A, Yoshikawa K (2006) Protoclusters in the $\Lambda$ CDM Universe. Astrophys J Lett 646:L5-L8. doi:10.1086/506607, arXiv:astro-ph/0606292

Tadhunter CN, Morganti R, Robinson A, Dickson R, Villar-Martín M, Fosbury RAE (1998) The nature of the optical-radio correlations for powerful radio galaxies. Mon Not Roy Astro Soc 298:1035-1047, astro-ph/9807238

Tananbaum H (2006) The Constellation X-ray Mission. Bull Am Astron Soci 38:393

Taniguchi Y, Shioya Y (2001) A new interpretation of chain galaxies at high redshift. Astrophys J 547:146153. doi:10.1086/318369, arXiv:astro-ph/0008379

Thomas D, Maraston C, Bender R, Mendes de Oliveira C (2005) The epochs of early-type galaxy formation as a function of environment. Astrophys J 621:673-694. doi:10.1086/426932, arXiv:astro-ph/0410209

Thompson D, Djorgovski S, Vigotti M, Grueff G (1994) Identifications and spectroscopy of faint radio galaxies from the B3 VLA survey. Astron J 108:828-836. doi:10.1086/117114

Tielens AGGM, Miley GK, Willis AG (1979) Westerbork observations of 4C sources with steep radio spectra. Astron \& Astrophys Suppl 35:153

Urry C (2004) AGN Unification: An Update. In: Richards GT, Hall PB (eds) AGN Physics with the Sloan Digital Sky Survey, Astronomical Society of the Pacific Conference Series, vol 311, pp 49

Urry CM, Padovani P (1995) Unified Schemes for Radio-Loud Active Galactic Nuclei. PASP 107:803, arXiv:astro-ph/9506063

Uson JM, Bagri DS, Cornwell TJ (1991) Radio detections of neutral hydrogen at redshift $Z=3.4$. Phys Rev Lett 67:3328-3331

van Breugel W, Heckman T, Butcher H, Miley G (1984) Extended optical line emission from 3C 293-Radio jets propagating through a rotating gaseous disk. Astrophys J 277:82-91. doi:10.1086/161673

van Breugel W, Filippenko AV, Heckman T, Miley G (1985a) Minkowski’s object - A starburst triggered by a radio jet. Astrophys J 293:83-93. doi:10.1086/163216

van Breugel W, Miley G, Heckman T, Butcher H, Bridle A (1985b) Optical emission-line gas associated with the radio source 3C 277.3. Astrophys J 290:496-500. doi:10.1086/163007 
van Breugel W, De Breuck C, Stanford SA, Stern D, Röttgering H, Miley G (1999) A Radio Galaxy at $Z=5$.19. Astrophys J Lett 518:L61-L64. doi:10.1086/312080, arXiv:astro-ph/9904272

van Breugel WJM, Dey A (1993) Induced star formation in a radio lobe of 3C 285? Astrophys J 414:563572. doi:10.1086/173103

van Breugel WJM, Stanford SA, Spinrad H, Stern D, Graham JR (1998) Morphological Evolution in HighRedshift Radio Galaxies and the Formation of Giant Elliptical Galaxies. Astrophys J 502:614. doi:10. 1086/305925, arXiv:astro-ph/9803019

van Groningen E, Miley GK, Norman CA (1980) One-sided jets in extragalactic radiosources. Astron \& Astrophys 90:L7-L9

van Ojik R, Rottgering HJA, Miley GK, Bremer MN, Macchetto F, Chambers KC (1994) TX0211-122: A starburst radio galaxy at $Z=2.34$. Astron \& Astrophys 289:54-60

van Ojik R, Röttgering HJA, Carilli CL, Miley GK, Bremer MN, Macchetto F (1996) A powerful radio galaxy at $z=3.6$ in a giant rotating Lyman $\alpha$ halo. Astron \& Astrophys 313:25-44, astro-ph/9608099

van Ojik R, Röttgering HJA, Miley GK, Hunstead RW (1997a) The gaseous environments of radio galaxies in the early Universe: kinematics of the Lyman $\alpha$ emission and spatially resolved $\mathrm{H}$ I absorption. Astron \& Astrophys 317:358-384, astro-ph/9608092

van Ojik R, Röttgering HJA, van der Werf PP, Miley GK, Carilli CL, Visser A, Isaak KG, Lacy M, Jenness T, Sleath J, Wink J (1997b) A search for molecular gas in high redshift radio galaxies. Astron \& Astrophys 321:389-396, arXiv:astro-ph/9610170

Venemans BP, Kurk JD, Miley GK, Röttgering HJA, van Breugel W, Carilli CL, De Breuck C, Ford H, Heckman T, McCarthy P, Pentericci L (2002) The Most Distant Structure of Galaxies Known: A Protocluster at $z=4.1$. Astrophys J Lett 569:L11-L14. doi:10.1086/340563, astro-ph/0203249

Venemans BP, Röttgering HJA, Overzier RA, Miley GK, De Breuck C, Kurk JD, van Breugel W, Carilli CL, Ford H, Heckman T, McCarthy P, Pentericci L (2004) Discovery of six Ly $\alpha$ emitters near a radio galaxy at $\mathrm{z} \sim$ 5.2. Astron \& Astrophys 424:L17-L20. doi:10.1051/0004-6361:200400041, astro-ph/0408520

Venemans BP, Röttgering HJA, Miley GK, Kurk JD, De Breuck C, Overzier RA, van Breugel WJM, Carilli CL, Ford H, Heckman T, Pentericci L, McCarthy P (2005) Properties of Ly $\alpha$ emitters around the radio galaxy MRC 0316-257. Astron \& Astrophys 431:793-812. doi:10.1051/0004-6361:20042038, astro-ph/0501259

Venemans BP, Röttgering HJA, Miley GK, van Breugel WJM, de Breuck C, Kurk JD, Pentericci L, Stanford SA, Overzier RA, Croft S, Ford H (2007) Protoclusters associated with z $>2$ radio galaxies . I. Characteristics of high redshift protoclusters. Astron \& Astrophys 461:823-845. doi:10.1051/0004-6361: 20053941, arXiv:astro-ph/0610567

Vernet J, Fosbury RAE, Villar-Martín M, Cohen MH, Cimatti A, di Serego Alighieri S, Goodrich RW (2001a) Erratum: Radio galaxies at $\mathrm{z} \sim 2.5$ : Results from Keck spectropolarimetry. Astron \& Astrophys 370:407-408. doi:10.1051/0004-6361:20010396

Vernet J, Fosbury RAE, Villar-Martín M, Cohen MH, Cimatti A, di Serego Alighieri S, Goodrich RW (2001b) Radio galaxies at z 2.5: Results from Keck spectropolarimetry. Astron \& Astrophys 366: 7-25. doi:10.1051/0004-6361:20000076, astro-ph/0010640

Villar-Martín M (2007) Giant Ly $\alpha$ nebulae in the high redshift (z > 2) Universe. New Astro Review 51:194-201. doi:10.1016/j.newar.2006.11.017, arXiv:astro-ph/0611763

Villar-Martín M, Binette L (1996) CA depletion and the presence of dust in large scale nebulosities in radiogalaxies. I. Astron \& Astrophys 309:97-108, astro-ph/9511113

Villar-Martín M, Binette L (1997) CA depletion and the presence of dust in large scale nebulosities in radiogalaxies. II. Astron \& Astrophys 317:350-357, astro-ph/9605195

Villar-Martín M, Binette L, Fosbury RAE (1996) The effects of resonance scattering and dust on the UV line spectrum of radio galaxies. Astron \& Astrophys 312:751-760, astro-ph/9605009

Villar-Martín M, Tadhunter C, Clark N (1997) The ionisation mechanism of the extended gas in high redshift radio galaxies: shocks or AGN photoionisation? Astron \& Astrophys 323:21-30, astro-ph/9701016

Villar-Martín M, Tadhunter C, Morganti R, Clark N, Killeen N, Axon D (1998) PKS 1932-464: a jet-cloud interaction in a radio galaxy? Astron \& Astrophys 332:479-492, astro-ph/9801039

Villar-Martín M, Binette L, Fosbury RAE (1999a) The nature of the extreme kinematics in the extended gas of high redshift radio galaxies. Astron \& Astrophys 346:7-12, astro-ph/9903122

Villar-Martín M, Tadhunter C, Morganti R, Axon D, Koekemoer A (1999b) PKS 2250-41 and the role of jetcloud interactions in powerful radio galaxies. Mon Not Roy Astro Soc 307:24-40, astro-ph/9901201 
Villar-Martín M, Alonso-Herrero A, di Serego Alighieri S, Vernet J (2000) Interpreting the kinematics of the extended gas in distant radiogalaxies from 8-10 m telescope spectra. Astron \& Astrophys Suppl 147:291-297, astro-ph/0009275

Villar-Martín M, De Young D, Alonso-Herrero A, Allen M, Binette L (2001) Shocks and dust survival in nearby active galaxies: implications for the alignment effect. Mon Not Roy Astro Soc 328:848-854. doi:10.1046/j.1365-8711.2001.04916.x, astro-ph/0108340

Villar-Martín M, Vernet J, di Serego Alighieri S, Fosbury R, Pentericci L, Cohen M, Goodrich R, Humphrey A (2002) Giant low surface brightness haloes in distant radio galaxies: USS0828 + 193. Mon Not Roy Astro Soc 336:436-444. doi:10.1046/j.1365-8711.2002.05751.x, arXiv:astro-ph/0206118

Villar-Martín M, Vernet J, di Serego Alighieri S, Fosbury R, Humphrey A, Pentericci L (2003) Kinematically quiet haloes around $\mathrm{z} \sim 2.5$ radio galaxies. Keck spectroscopy. Mon Not Roy Astro Soc 346:273-294. doi:10.1046/j.1365-2966.2003.07090.x, astro-ph/0309012

Villar-Martín M, Tadhunter C, Morganti R, Holt J (2005) The giant star forming halo associated with the radio galaxy PKS 1932-46. Mon Not Roy Astro Soc 359:L5-L9. doi:10.1111/j.1745-3933.2005. 00022.x, astro-ph/0501414

Villar-Martín M, Gómez JL, González Delgado R, Pérez E (eds) (2006a) The Granada workshop on High Redshift Radio Galaxies, vol 327

Villar-Martín M, Sánchez SF, De Breuck C, Peletier R, Vernet J, Rettura A, Seymour N, Humphrey A, Stern D, di Serego Alighieri S, Fosbury R (2006b) VIMOS-VLT and Spitzer observations of a radio galaxy at $z=2.5$. Mon Not Roy Astro Soc 366:L1-L5. doi:10.1111/j.1745-3933.2005.00118.x, astro-ph/0510733

Villar-Martín M, Humphrey A, De Breuck C, Fosbury R, Binette L, Vernet J (2007a) Ly $\alpha$ excess in highredshift radio galaxies: a signature of star formation. Mon Not Roy Astro Soc 375:1299-1310. doi:10. 1111/j.1365-2966.2006.11371.x, astro-ph/0612116

Villar-Martín M, Sánchez SF, Humphrey A, Dijkstra M, di Serego Alighieri S, De Breuck C, González Delgado R (2007b) VIMOS-VLT spectroscopy of the giant Ly $\alpha$ nebulae associated with three $\mathrm{z} \sim$ 2.5 radio galaxies. Mon Not Roy Astro Soc 378:416-428. doi:10.1111/j.1365-2966.2007.11811.x, arXiv:0704.1116

Weidinger M, Møller P, Fynbo JPU (2004) The Lyman- $\alpha$ glow of gas falling into the dark matter halo of a $z=3$ galaxy. Nature 430:999-1001. doi:10.1038/nature02793, arXiv:astro-ph/0408478

Weidinger M, Møller P, Fynbo JPU, Thomsen B (2005) The extended Lyman- $\alpha$ emission surrounding the $z=3.04$ radio-quiet QSO1205-30: Primordial infalling gas illuminated by the quasar? Astron \& Astrophys 436:825-835. doi:10.1051/0004-6361:20042304, arXiv:astro-ph/0503241

White SDM, Rees MJ (1978) Core condensation in heavy halos - A two-stage theory for galaxy formation and clustering. Mon Not Roy Astro Soc 183:341-358

Willott CJ, Rawlings S, Blundell KM, Lacy M, Eales SA (2001) The radio luminosity function from the low-frequency 3CRR, 6CE and 7CRS complete samples. Mon Not Roy Astro Soc 322:536-552, arXiv:astro-ph/0010419

Willott CJ, Rawlings S, Blundell KM, Lacy M, Hill GJ, Scott SE (2002) Optical spectroscopy of radio galaxies in the 7C Redshift Survey. Mon Not Roy Astro Soc 335:1120-1132. doi:10.1046/j.1365-8711. 2002.05718.x, arXiv:astro-ph/0205507

Wilman RJ, Jarvis MJ, Röttgering HJA, Binette L (2004) HI in the protocluster environment at z > 2: absorbing haloes and the Ly $\alpha$ forest. Mon Not Roy Astro Soc 351:1109-1119. doi:10.1111/j.1365-2966. 2004.07857.x, arXiv:astro-ph/0403519

Windhorst RA, Burstein D, Mathis DF, Neuschaefer LW, Bertola F, Buson LM, Koo DC, Matthews K, Barthel PD, Chambers KC (1991) The discovery of a young radio galaxy at $Z=2.390$ —Probing initial star formation at Z less than approximately 3.0. Astrophys J 380:362-383. doi:10.1086/170596

Yuan W, Fabian AC, Celotti A, Jonker PG (2003) Extended X-ray emission in the high-redshift quasar GB $1508+5714$ at $z=4.3$. Mon Not Roy Astro Soc 346:L7-L10. doi:10.1046/j.1365-2966.2003.07234. $\mathrm{x}$, arXiv:astro-ph/0309318

Zirm AW, Overzier RA, Miley GK, Blakeslee JP, Clampin M, De Breuck C, Demarco R, Ford HC, Hartig GF, Homeier N, Illingworth GD, Martel AR, Röttgering HJA, Venemans B, Ardila DR, Bartko F, Benítez N, Bouwens RJ, Bradley LD, Broadhurst TJ, Brown RA, Burrows CJ, Cheng ES, Cross NJG, Feldman PD, Franx M, Golimowski DA, Goto T, Gronwall C, Holden B, Infante L, Kimble RA, Krist JE, Lesser MP, Mei S, Menanteau F, Meurer GR, Motta V, Postman M, Rosati P, Sirianni M, Sparks WB, Tran HD, Tsvetanov ZI, White RL, Zheng W (2005) Feedback and brightest cluster galaxy formation: ACS observations of the radio galaxy TN J1338-1942 at $z=4$.1. Astrophys J 630:68-81. doi:10.1086/431921, astro-ph/0505610 